\title{
Effect of parent body evolution on equilibrium and kinetic isotope fractionation: a combined Ni
} and Fe isotope study of iron and stony-iron meteorites

\author{
Stepan M. Chernonozhkin ${ }^{\mathrm{a}, \mathrm{b}}$, Steven Goderis ${ }^{\mathrm{a}, \mathrm{b}}$, Marta Costas-Rodríguez ${ }^{\mathrm{a}}$, Philippe Claeys ${ }^{\mathrm{b}}$ and Frank \\ Vanhaecke $\mathrm{a}^{\mathrm{a}^{*}}$ \\ ${ }^{a}$ Ghent University, Department of Analytical Chemistry, Krijgslaan, 281 - S12, 9000 Ghent, Belgium. E- \\ mail: Frank.Vanhaecke@UGent.be \\ ${ }^{\mathrm{b}}$ Vrije Universiteit Brussel, Analytical-, Environmental-, and Geo-Chemistry, Pleinlaan 2, 1050 Brussels, \\ Belgium
}

\begin{abstract}
Various iron and stony-iron meteorites have been characterized for their $\mathrm{Ni}$ and $\mathrm{Fe}$ isotopic compositions using multi-collector inductively coupled plasma-mass spectrometry (MC-ICP-MS) after sample digestion and chromatographic separation of the target elements in an attempt to further constrain the planetary differentiation processes that shifted these isotope ratios and to shed light on the formational history and evolution of selected achondrite parent body asteroids. Emphasis was placed on spatially resolved isotopic analysis of iron meteorites, known to be inhomogeneous at the $\mu \mathrm{m}$ to $\mathrm{mm}$ scale, and on the isotopic characterization of adjacent metal and silicate phases in main group pallasites (PMG), mesosiderites, and the IIE and IAB complex silicate-bearing iron meteorites. In a 3 -isotope plot of ${ }^{60 / 58} \mathrm{Ni}$ versus ${ }^{62 / 58} \mathrm{Ni}$, the slope of the best-fitting straight line through the laterally resolved $\mathrm{Ni}$ isotope ratio data for iron meteorites reveals kinetically controlled isotope fractionation $\left(\beta_{\text {exper }}=1.981 \pm 0.039,1 \mathrm{SD}\right)$, predominantly resulting from sub-solidus diffusion (with the fractionation exponent $\beta$ connecting the isotope fractionation factors, as $\alpha_{62 / 58}=\alpha_{60 / 58}{ }^{\beta}$ ). The observed relation between $\delta^{56 / 54} \mathrm{Fe}$ and Ir concentration in the metal fractions of PMGs and in IIIAB iron meteorites indicates a dependence of the bulk $\mathrm{Fe}$ isotopic composition on the fractional crystallization of an asteroidal metal core. No such fractional crystallization trends were found for the corresponding $\mathrm{Ni}$ isotope ratios or for other iron meteorite groups, such as the IIABs. In the case of the IIE and IAB silicate-bearing iron meteorites, the Fe and Ni isotopic signatures potentially reflect the influence of impact processes, as the degree of diffusion-controlled Ni isotope fractionation is closer to that of Fe compared to what is observed for magmatic iron meteorite types. Between the metal and olivine counterparts of pallasites, the $\mathrm{Fe}$ and $\mathrm{Ni}$ isotopic compositions show clearly resolvable differences, similar in magnitude but opposite in sign $\left(\Delta^{56 / 54} \mathrm{Fe}_{\text {met-oliv }}\right.$ of $+0.178 \pm 0.092 \%$ and $\Delta^{60 / 58} \mathrm{Ni}_{\text {met-oliv }}$ of $0.212 \pm 0.082 \%, 2 \mathrm{SD})$. As such, the heavier Fe isotope ratios for the metal $\left(\delta^{56 / 54} \mathrm{Fe}=+0.023\right.$ to $+0.247 \%$ ) and lighter values for the corresponding olivines $\left(\delta^{56 / 54} \mathrm{Fe}=-0.155\right.$ to $-0.075 \%$ ) are interpreted to reflect later-stage Fe isotopic re-equilibration between these phases, rather than a pristine record of mantle-core differentiation. In the case of mesosiderites, the similarly lighter $\mathrm{Ni}$ and $\mathrm{Fe}$ isotopic signatures found for the silicate phase $(-0.149$ to $+0.023 \%$ for $\delta^{60 / 58} \mathrm{Ni},-0.214$ to $-0.149 \%$ for $\left.\delta^{56 / 54} \mathrm{Fe}\right)$ compared to the metal phase $(+0.168$ to $+0.191 \%$ for $\delta^{60 / 58} \mathrm{Ni},+0.018$ to $+0.120 \%$ for $\delta^{56 / 54} \mathrm{Fe}$ ) likely result from $\mathrm{Fe}$ and $\mathrm{Ni}$ diffusion. Overall, the $\mathrm{Fe}$ and $\mathrm{Ni}$ isotopic compositions of iron-rich meteorites reflect multiple, often superimposed, processes of equilibrium or kinetic nature, illustrating convoluted parent body histories and late-stage interaction between early-formed planetesimal reservoirs.
\end{abstract}


Keywords: Fe and Ni isotopic composition; pallasites; mesosiderites; iron meteorites; mass-dependent 2 isotope fractionation; core formation 


\section{Introduction}

At present, natural stable isotope fractionation has been shown to affect elements with $\geq 2$ isotopes. Generally, differences of the order of $\sim 1 \%$ are observed for the isotope ratios on the bulk meteorite scale, with usually even larger effects for lighter elements. The overwhelming part of these isotope fractionations exhibit a mass-dependent character, indicating that the extent of the observed isotope fractionation, caused by chemical reaction or phase change, depends on the masses of the isotopes. Based on the underlying natural process, the distinction is made between equilibrium and kinetic mass-dependent fractionation. Equilibrium mass-dependent isotope fractionation occurs during exchange between compounds (or between phases) as a result of differences in the equilibrium reaction constants for different isotopes, which arise from slightly different vibrational energy levels for the molecules that contain these different isotopes. As such, equilibrium isotope fractionation largely depends on the bonding environment of the atom and the temperature of the governing process. In contrast, kinetic mass-dependent isotope fractionation arises without achieving equilibrium in the system, as a result of the different rates at which the isotopes with different masses are involved in a process (either physical rates, as in the case of diffusion, or chemical reaction rates). A classical example of kinetic isotope fractionation is the irreversible evaporation to the vacuum of an element, where the rate constants depend on the isotopic masses (Alexander and Wang, 2001; Dauphas et al., 2004; F. Richter et al., 2009). For ordinary chondrites, isotopic variations of $\mathrm{Ni}, \mathrm{Cu}$ and $\mathrm{Zn}$ have been suggested to result from vapor-solid reactions, followed by mineral sorting during accretion (Moynier et al, 2007). Similarly, the isotope ratios of the elements taking part in chemical or thermal diffusion can be affected, due to the fact that isotopes tend to diffuse at dissimilar rates as a result of differences in their masses (F. M. Richter et al., 2009). Equilibration of the isotope ratios between two adjacent phases is achieved via diffusive isotopic exchange, which, in its turn, is associated with kinetic isotope effects. During slow cooling of mineral assemblages, diffusion will effectively stop at a particular temperature. In some cases, when equilibrium cannot be achieved, some degree of the kinetic control on the isotope ratios will be preserved, dependent on, for instance, the cooling rate, the target element and the mineral assemblage studied (e.g. Mueller et al. 2010). In practice, the distinction between kinetic and equilibrium mass-dependent isotope effects can be made by calculating the theoretical fractionation exponents $\beta$ for equilibrium and kinetic isotope control, and by comparing these to the experimental slope obtained from the corresponding 3-isotope plot (Young et al. 2002):

$$
\begin{aligned}
& \beta_{e q}=\frac{\left(1 / m_{58}-1 / m_{62}\right)}{\left(1 / m_{58}-1 / m_{60}\right)} \\
& \beta_{\text {kin }}=\frac{\ln \left(m_{58} / m_{62}\right)}{\ln \left(m_{58} / m_{60}\right)}
\end{aligned}
$$

In the Early Solar System, the intense heating of initially undifferentiated planetesimals as a result of short-lived $\beta^{-}$isotope decay of ${ }^{26} \mathrm{Al}$ led to the differentiation of these bodies with the formation of silicatedominated mantles and iron-rich cores, from which iron-rich achondritic meteorites derive (Grimm and Mcsween, 1993). These planetary fractionation processes not only affected the elemental composition, but also the isotopic composition of the meteorite parent bodies (Moynier et al., 2007). In the case of iron-rich meteorites, $\mathrm{Fe}$ and $\mathrm{Ni}$ can experience resolvable equilibrium isotope fractionation in the processes associated with the melting of undifferentiated precursors and subsequent segregation of metal from silicate. The degree of equilibrium isotope fractionation between the co-existing phases depends on the temperature at which these 
processes occurred. Under the temperature and pressure conditions in asteroid-size parent bodies, heavier $\mathrm{Fe}$ isotopic compositions for the metal core and lighter isotope ratios for silicate phases relative to the initial isotopic composition of the precursor material are expected (Polyakov, 2009). Trace element concentration trends within most iron meteorite groups reflect fractional crystallization (Scott, 1972; Goldstein et al., 2009), which confirms the formation of $\sim 2$ to $100 \mathrm{~km}$ sized metallic cores within differentiated meteorite parent bodies (Chabot and Haack, 2006). However, another class of iron meteorites, known as the IAB complex and IIE silicate-bearing groups (previously designated "non-magmatic"), shows no compositional trends indicative of fractional crystallization. Instead, these meteorites contain enclosed silicates and are interpreted to have formed as a result of planetesimal collision. For instance, the IAB meteorite complex is thought to result from impact on a partially differentiated asteroid that experienced less peak heating and in part preserved a chondritic composition (Goldstein et al., 2009; Ruzicka, 2014). If this presumed model is correct, the expected Fe-Ni isotopic variations in silicate-bearing iron meteorites arising due to impact processing should be different from those resulting from magmatic processing and core formation in the purely magmatic iron meteorites groups. Impact processing potentially includes preferential evaporation of light isotopes in a process similar to the modeled evaporation to vacuum, with an associated shift to heavier isotopic compositions. In contrast to magmatic processes, for which $\mathrm{Fe}$ and $\mathrm{Ni}$ isotope ratios depend on crystal chemistry constraints and on the corresponding fractions that went into the silicate and metal fractions, the relative isotope ratio shifts for $\mathrm{Fe}$ and $\mathrm{Ni}$ in the case of impact should be of similar magnitude, given the comparable element volatilities. $\mathrm{CB}$ chondrites are thought to have some components that formed by condensation in impact plume (Tang and Dauphas, 2012; Richter et al., 2014; Weyrauch et al., 2015).

However, later-stage processes leading to $\mathrm{Fe}$ and $\mathrm{Ni}$ isotope fractionation in iron meteorite parent bodies, such as sub-solidus diffusion during the Widmanstätten pattern growth, also need to be considered. This diffusion results from the growth of Ni-poor kamacite (bcc $\alpha$-iron) from taenite (fcc $\gamma$-iron) during the slow (a few to thousands ${ }^{\circ} \mathrm{C} \mathrm{Myr}^{-1}$; (Goldstein et al., 2014) cooling of iron meteorite parent bodies and the dissimilar diffusivities for the different isotopes. During cooling, initially stable taenite decomposes to form intergrown kamacite and taenite lamellae via solid state diffusion of $\mathrm{Ni}$ and $\mathrm{Fe}$ atoms, a process that is accompanied by kinetic isotope fractionation. The fractionation of $\mathrm{Fe}$ and $\mathrm{Ni}$ isotopes during the growth of kamacite out of taenite during the formation of the Widmanstätten pattern has been modeled for a set of cooling rates ranging from 25 to $500{ }^{\circ} \mathrm{C} \mathrm{Myr}^{-1}$ (Dauphas, 2007). As equilibrium fractionation of $\mathrm{Fe}$ and $\mathrm{Ni}$ isotopes due to the redistribution between $\alpha$ and $\gamma$ phases following crystal chemistry constraints is also possible, the contributions of kinetic and equilibrium isotope fractionation need to be evaluated. The cooling histories of the iron meteorite parent bodies are reflected in the metallographic cooling rates determined for the iron meteorite group members. For instance, IVA iron meteorites are characterized by a wide range of cooling rates between 100 and $6600{ }^{\circ} \mathrm{C} \mathrm{Myr}^{-1}\left(1500{ }^{\circ} \mathrm{C}\right.$ $\mathrm{Myr}^{-1}$ for Gibeon) (Goldstein et al. 2014). These cooling rates depend on the size of the parent body, the depth within the core and prevalence and thickness of any covering mantle.

Stony-iron meteorites, consisting of pallasites and mesosiderites, are closely related to iron meteorites. Both meteorite groups are composed of nearly equal parts of metal, derived from the metal core of a differentiated asteroid, and a silicate counterpart. This silicate is made up of mantle-derived olivine crystals in the case of pallasites or brecciated remains of basaltic minerals of predominantly crustal origin in the case of mesosiderites. Besides the observation that the parent bodies of both meteorite groups show extensive evidence 
for processes related to impact and collision, there is no data to support a common origin from the same parent body (Greenwood et al., 2006). For instance, the $\Delta^{17} \mathrm{O}$ oxygen isotopic compositions of olivines in pallasites and mesosiderites differ by $\sim 0.06 \%$ (Greenwood et al., 2015). An alternative hypothesis for mesosiderite formation envisions mixing of brecciated basaltic/pyroxenic silicates (Ruzicka et al., 1994) derived from igneous differentiated asteroid crust/regolith with asteroidal core fragments upon impact (Wasson and Rubin, 1985; Hassanzadeh et al., 1990). Although the exact formational mechanism of pallasites is still heavily debated, a model involving collision with a substantially molten parent body, which separated a partially solidified core and mantle containing olivine and silicate melt, remains a compelling possibility (Ruzicka, 2014). In a "hit and run" impact scenario, molten metal and silicate mantle material from the core-mantle boundary would be mixed and re-accreted (Yang et al., 2010). Poitrasson et al. (Poitrasson et al., 2005) reported heavy Fe isotopic compositions for the metal part of pallasites $\left(\delta^{57 / 54} \mathrm{Fe}\right.$ of -0.020 to $+0.237 \%$ ) and light Fe isotopic compositions for the corresponding olivine fractions $\left(\delta^{57 / 54} \mathrm{Fe}\right.$ of -0.090 to $+0.021 \%$ ). If the conditions for Fe isotopic equilibration between the adjacent olivine and metal phases are fulfilled, the resulting $\mathrm{Fe}$ isotope ratio signatures can be used as an isotope geothermometer, relying on the temperature dependence of the distribution of Fe isotopes between the phases (Polyakov 2009, Dauphas et al. 2012). However, it remains unclear whether the olivine-metal $\mathrm{Fe}$ isotope equilibration and the corresponding temperatures were fully achieved in the process of initial metal-silicate segregation of the parent body, or if such equilibrium was altered during later reequilibrating thermal events.

In this work, new stable $\mathrm{Fe}$ and $\mathrm{Ni}$ isotope ratio data are provided for 4 pallasites and 4 magmatic iron meteorites, permitting one to study the isotope fractionation of these elements between adjacent silicate-metal and metal-metal phases and to provide insight into the formation history and evolution of their parent bodies. Stable isotope fractionation of $\mathrm{Fe}$ and $\mathrm{Ni}$ was also studied in 3 mesosiderites and 5 silicate-bearing iron meteorites, including IAB-ung Udei Station. Although the stable Fe and Ni isotope signatures of these meteorites are not related to core formation processes, they could potentially reveal links with the cooling histories of these meteorite parent bodies, or late-stage collision events. Different to previous work, the current study focuses on the isotopic analysis of both $\mathrm{Ni}$ and the relatively well-understood $\mathrm{Fe}$, of the same meteorite sub-samples digestions. This strategy provides additional constraints on the processes that lead to Fe-peak element stable isotope fractionation. The previously studied occurrence of nucleosynthetic anomalies or the radiogenic ingrowth of Ni isotopes are not addressed in this work (Dauphas et al., 2008; Regelous et al., 2008; Steele et al., 2011; Tang and Dauphas, 2014). 


\section{Experimental}

\subsection{Samples}

In total, 4 iron meteorite specimens (Henbury IIIAB, Campo del Cielo IAB-MG, Gibeon IVA and Canyon Diablo IAB-MG) as well as 3 main group pallasites (Seymchan, Fukang and Esquel) were purchased from commercial meteorite vendors. Four iron meteorites (Chinga IVB-an, Sikhote-Alin IIB, Elga IIE and Darinskoe IIC) were obtained from the Central Siberian Geological Museum, V. S. Sobolev Institute of Geology and Mineralogy, Russia. A selection of these iron meteorites were characterized for major and trace element concentrations in a previous work (Chernonozhkin et al., 2014). Three mesosiderites, EET 87500, RKPA 79015 and QUE 93001 and main group pallasite CMS 04071 were provided from the Antarctic meteorite collection by the National Aeronautics and Space Administration (NASA). Three ordinary chondrites A 10224 (L3), A 09135 (LL3) and A 09436 (H3) were provided by the Royal Belgian Institute of Natural Sciences (RBINS) from their Antarctic meteorite collection. OKUM is a certified reference material of an ultramafic komatiite (Geosciences Laboratories, Sudbury, Ontario, Canada). PCC-1 and BHVO-1 are certified reference materials of peridotite and basalt (United States Geological Survey, Denver, Colorado, USA).

\subsection{Reagents and labware}

Only high-purity reagents and acids were used throughout the experiments. Pro analysis grade nitric acid (65\%, Chem-Lab, Belgium) was further purified through sub-boiling in PFA equipment. Optima-grade hydrochloric (37\%, Seastar Chemicals Inc., Canada) and trace-metal grade hydrofluoric (47-51\%, Seastar Chemicals Inc., Canada) acids were used as such. Ultrapure water (resistivity $\geq 18.2 \mathrm{M} \Omega \mathrm{cm}$ ) was obtained from a Milli-Q Element water purification system (Millipore, France). Purissima grade acetone, ultrapure $9.8 \mathrm{M} \mathrm{H}_{2} \mathrm{O}_{2}$ and dimethylglyoxime (DMG) were purchased from Sigma Aldrich (Belgium). Savillex ${ }^{\mathrm{TM}}$ Teflon ${ }^{\circledR}$ beakers were pre-cleaned by alternating $24 \mathrm{~h}$ soaking steps in pro-analysis $6 \mathrm{M} \mathrm{HCl}$ and $7 \mathrm{M} \mathrm{HNO}_{3}$ at $110^{\circ} \mathrm{C}(4$ times). All the Teflon ${ }^{\circledR}$ recipients, disposable plastic tubes and pipette tips used for the sample preparation were additionally cleaned by soaking in $10 \%$ pro-analysis $\mathrm{HCl}$ for $48 \mathrm{~h}$ at $110^{\circ} \mathrm{C}$. The chromatographic isolation of the target elements, hot-plate evaporation and labware cleaning procedures were performed under class-10 clean lab conditions to reduce airborne contamination.

\subsection{Sub-sampling and sample preparation}

The allocated mesosiderite chips were first broken into smaller pieces and manually ground in an agate mortar that was pre-cleaned by grinding pro-analysis quartz grains to powder 3 times, rinsing with ultrapure water, and drying. The ground mesosiderites were separated into non-magnetic (150-200 mg) and magnetic (350-600 mg) fractions using a hand magnet. These fractions are called silicate and metal fractions, respectively, hereafter, although this magnetic fraction might contain magnetic species other than metal, such as oxides. A diamond disk saw was used to cut 100-200 mg fragments from the bulk mass of the pallasite metal. Visibly oxidized surfaces were removed by cleaning with silicon carbide sandpaper, etching with dilute $\mathrm{HNO}_{3}$ in an ultrasonic bath, and finally rinsing with ethanol to avoid contamination of the surface. The olivine crystals (200$300 \mathrm{mg}$ ) closely attached to the sampled metal parts were split from the pallasites using stainless steel tweezers. The olivine crystals were collected into an agate mortar and manually ground with an agate pestle. Any remaining minor metal was removed using a hand magnet. 
Five iron meteorites (Gibeon, Henbury, Canyon Diablo, Campo del Cielo and Chinga) were further subsampled via computer-assisted micro-drilling under an optical microscope. Sampling positions for 3 of these meteorites are shown in the two-dimensional (2D) distribution maps of the $\mathrm{Ni} \mathrm{K}_{\alpha}$ intensity obtained by micro Xray fluorescence spectrometry $(\mu \mathrm{XRF})$ (Figure 1A, B and C). The uniform distribution maps obtained for Campo del Cielo and Chinga are not shown. Sampling pure taenite phases was difficult because the drill bit diameter is larger than the taenite lamellae. In addition, drilling can mix phases vertically. As a result, drilling resulted in phase mixtures characterized by different taenite/kamacite ratios. In the case of Canyon Diablo, bright Ni-rich veinlets were not attributed to taenite, as they also contain elevated contents of P. These veinlets were avoided during sampling.

Approximately $400 \mu \mathrm{m}$ deep pits were drilled in a polished thick section of the iron meteorites at 1750 $\mathrm{rpm}$ in the presence of a $10 \mu \mathrm{l}$ droplet of ultrapure water. The wet sample powder was collected with a pipet into a Savillex ${ }^{\mathrm{TM}}$ Teflon ${ }^{\circledR}$ beaker. The surface of the sample was cleaned with ethanol under a microscope after each spot drilling to avoid cross-contamination. The potential contamination from the material of the drill bit (tungsten carbide, WC) was controlled by monitoring the $\mathrm{W}$ content in the digested samples using sector field ICP-MS (SF-ICP-MS). The presence of W, predominantly present in the WC drill bit, and relatively depleted in iron meteorite material, was either below the detection limit, or negligible compared to the contents of Fe and $\mathrm{Ni}$. As such, any contamination of meteorite material with $\mathrm{Fe}$ and $\mathrm{Ni}$ from the drill bit is considered to be insignificant. In addition, to evaluate potential isotope fractionation resulting from melting that may occur during the drilling, several micro-drillings of the Chinga IVB homogeneous ataxite were sampled and the resulting Fe and $\mathrm{Ni}$ isotope ratios were compared to bulk sample measurements. As the results were indistinguishable within analytical uncertainty, isotope fractionation during micro-drilling was considered to be insignificant. The ordinary chondrite material (50-100 mg) was sampled by drilling multiple 1-2 mm deep holes from the inside of the samples provided using a $1 \mathrm{~mm}$ diameter WC drill bit at low rotation speeds. The fusion crusts of the same samples were collected separately $(10-20 \mathrm{mg})$, by drilling shallow $(0.5-1 \mathrm{~mm})$ spots at the exterior of these meteorites.

The metal fractions of pallasites and mesosiderites were digested using $6 \mathrm{ml}$ of freshly prepared aqua regia $\left(1.5 \mathrm{ml} \mathrm{HNO}_{3}\right.$ and $\left.4.5 \mathrm{ml} \mathrm{HCl}\right)$ in closed Savillex ${ }^{\mathrm{TM}}$ Teflon ${ }^{\circledR}$ beakers at $90^{\circ} \mathrm{C}$ for $96 \mathrm{~h}$. Micro-drilled subsamples of iron meteorites were digested in a similar way using $1 \mathrm{ml}$ of aqua regia only. As the metal fractions of mesosiderites were not digested completely (silicate particles might have been captured during magnetic separation), centrifugation was used to separate off the residue. The chondrites and the silicate counterparts of stony-iron meteorites were first digested in $3 \mathrm{ml}$ of $14 \mathrm{M} \mathrm{HNO}_{3}$ at $90^{\circ} \mathrm{C}$ for $24 \mathrm{~h}$ in closed Savillex ${ }^{\mathrm{TM}} \mathrm{Teflon}{ }^{\circledR}$ beakers. Subsequently, the mixtures thus obtained were transferred into microwave Teflon vials and further digested via a two-step microwave-assisted acid digestion. In a first step, a combination of $\mathrm{HF}$ and $\mathrm{HNO}_{3}$ is used to break down the silicates, while, following evaporation to dryness at $90^{\circ} \mathrm{C}$, the digestion is completed in aqua regia to dissolve insoluble fluorides (Chernonozhkin et al., 2015). Finally, all solutions were evaporated to incipient dryness in open vessels at $90^{\circ} \mathrm{C}, 0.1 \mathrm{ml}$ of $12 \mathrm{M} \mathrm{HCl}$ was added to all the digests, and the solutions were evaporated again to dryness at $90^{\circ} \mathrm{C}$. This last step was repeated 3 times to convert all nitrates to chlorides before the isolation procedure. The meteorite digests were divided in 3 parts to perform $i$ ) element analysis, ii) isotopic analysis of $\mathrm{Ni}$, and iii) isotopic analysis of Fe. The bulk digestions of iron meteorites, previously used for $\mathrm{Ni}$ isotopic analysis (Chernonozhkin et al., 2015), were used in this study to determine Fe isotope ratios. 
Small aliquots of the digested meteorites were taken and diluted in $3 \% \mathrm{HNO}_{3}$ for the quantitative determination of the target elements using SF-ICP-MS.

The $\mathrm{Ni}$ isolation from the iron and silicate matrices was carried out using a 3-step ion exchange chromatographic procedure, previously described in detail (Chernonozhkin et al., 2015). In a first step, Ni is separated from the main part of the matrix using Dowex 50WX4 cation exchange resin in a medium of $\mathrm{HCl}$ acetone-dimethylglyoxime. A second step utilizes a column packed with AG1X8 anion exchange resin in HClHF medium to separate Ni from Ti, while a third anion exchange isolation step is used for final clean-up of the $\mathrm{Ni}$ fraction from traces of $\mathrm{Fe}$ and $\mathrm{Zn}$ in $6 \mathrm{M} \mathrm{HCl}$. The Fe isolation from the iron meteorites and silicate matrices relies on anion exchange column chromatography using AG MP-1 strong anion exchange resin in $\mathrm{HCl}$ medium. An adaptation of the chromatographic isolation method first described for geological samples was utilized (Maréchal et al., 1999; Van Heghe et al., 2012). The Ni and Fe concentrations were also determined in the isolated fractions to verify quantitative recoveries and the purity of the fractions.

\subsection{Instrumentation and measurement protocols}

The 2D element distribution maps of the thick iron meteorite sections were obtained using a Bruker M4 Tornado XRF instrument at the Vrije Universiteit Brussel, equipped with a 30W Rh-anode X-ray source $(50 \mathrm{kV}$ operating voltage, operating current $150 \mu \mathrm{A}$ ) and a Be side-window. The X-rays are focused to a $25 \mu \mathrm{m}$ spot (measured for Mo $\mathrm{K} \alpha$ ) using a polycapillary lens. The instrument is equipped with two Silicon Drift Detector (SDD) spectrometers $\left(30 \mathrm{~mm}^{2}, 145 \mathrm{eV}\right.$ energy resolution for $\left.\mathrm{Mn} \mathrm{Ka}\right)$, symmetrically placed relative to the sample. Measurements were performed under a vacuum of 20 mbar. To acquire the 2D elemental distribution maps, a motorized sample stage moves at a speed of $25 \mathrm{~mm} \mathrm{~s}^{-1}$ (1 ms per pixel), scanning the surface for 8 cycles.

The 2D element distribution maps of pallasite olivines were obtained using a $193 \mathrm{~nm}$ Teledyne Cetac Technologies Photon Machines Analyte G2 ArF* excimer-based laser ablation (LA) system with a doublevolume ablation cell coupled to a SF-ICP-MS unit (see below) at Ghent University. The maps were acquired at 9 $\mu \mathrm{m} \mathrm{s}^{-1}$ translation speed, $20 \mu \mathrm{m}$ spot diameter, $3.54 \mathrm{~J} \mathrm{~cm}^{-2}$ energy fluence, and covered a square area of 1450x600 $\mu \mathrm{m}$ from the metal-olivine margin in the direction of the olivine cores. The He carrier gas was mixed with the Ar sample gas downstream of the ablation cell, and introduced into the ICP-MS unit, operated at low mass resolution and cold plasma conditions. Wash-out times were typically less than $1 \mathrm{~s}$. The signal was acquired in the "speed mode" of the instrument, at $1.94 \mathrm{~s}$ per scan total scanning speed. The spatial resolution achieved was approximately $20 \times 20 \mu \mathrm{m}$. Quantification relied on a combination of external calibration (versus the synthetic glasses NIST SRM 612, NIST SRM 614 and artificial and natural CRM glasses from the USGS GSD-1G, GSE-1G, BHVO-2G and BIR-1G) and internal sum normalization using the intensities of Mg, Fe and $\mathrm{Si}$ and relying on the empirical formula of the olivine mineral.

A solid sampling system Merchantek MicroMill equipped with a drill mounted on a video microscope assembly and a set of motorized stages was used for the micro-drilling and milling of the samples at the Vrije Universiteit Brussel. A $300 \mu \mathrm{m}$ diameter WC drill bit (Komet dental) was used.

For the acid digestion of the samples, a MLS-1200 MEGA Microwave digestion system (Milestone, Italy) was used at Ghent University.

Element concentrations were determined using a Thermo Scientific Element XR SF-ICP-MS unit operated in low and medium resolution modes, using In as an internal standard, at Ghent University. 
The isotopic analysis of $\mathrm{Ni}$ and $\mathrm{Fe}$ was performed in separate measurement sessions using a Thermo Scientific Neptune multi-collector ICP-MS (MC-ICP-MS) unit equipped with a jet interface and dual spray chamber, consisting of a cyclonic and a Scott-type sub-unit, with a $100 \mu 1 \mathrm{~min}^{-1}$ concentric nebulizer in medium resolution mode. For the detailed description of the instrument settings, the reader is referred to the electronic supplementary information (ESI). Measurement of $\mathrm{Ni}$ and Fe isotope ratios was performed in a sample-standard bracketing (SSB) sequence relative to the NIST SRM 986 and IRMM-014 isotopic reference materials, respectively. The concentrations of $\mathrm{Fe}$ and $\mathrm{Ni}$ were adjusted to $400 \mathrm{ng} \mathrm{g}^{-1}$ using $3 \% \mathrm{HNO}_{3}$ and doped with $\mathrm{Cu}$ and Ni internal standards, respectively. Mass bias correction relied on the internal isotopic standard, according to the Russell equation revised by Baxter et al. 2006, which corrects for both the mass- and time-dependent functional parts of the instrumental mass discrimination. After the internal mass bias correction, the isotope ratios are reported as delta values, relative to the bracketing isotopic reference material to correct for minor drift of the instrumental parameters:

$$
\begin{aligned}
& \delta^{x / 58} \mathrm{Ni}=\left[\frac{\left({ }^{x} \mathrm{Ni} /{ }^{58} \mathrm{Ni}\right)_{\text {Cu corr }, \text { smp }}}{\left({ }^{x} \mathrm{Ni} /{ }^{58} \mathrm{Ni}\right)_{\text {Cu corr }, \text { NISTSRM } 986}}-1\right] \cdot 1,000 \\
& \delta^{x / 54} \mathrm{Fe}=\left[\frac{\left({ }^{x} \mathrm{Fe} /{ }^{54} \mathrm{Fe}\right)_{\mathrm{Ni} \text { corr }, \text { smp }}}{\left({ }^{x} \mathrm{Fe} /{ }^{54} \mathrm{Fe}\right)_{\mathrm{Ni} \text { corr }, \text { IRMM } 014}}-1\right] \cdot 1,000
\end{aligned}
$$

where $x$ is $60,61,62$ or 64 for $\mathrm{Ni}$ and 56 or 57 for $\mathrm{Fe}$, and the subscripts denote that the ratios were first corrected by an exponential model. For further details, the reader is referred to previous publications of our group (Van Heghe et al., 2012; Chernonozhkin et al., 2015). 
The Ni concentration of and 2D distribution in various meteorites (Gibeon, Henbury, Canyon Diablo, Campo del Cielo and Chinga) were characterized using $\mu$ XRF. Among these meteorites, Gibeon shows the widest taenite lamellae, less than $0.5 \mathrm{~mm}$ in width, with distinctive M-shaped Ni concentration profiles reaching a maximum of $11.06-12.22 \mathrm{wt} \% \mathrm{Ni}$ (Figure 1), with 6.09-6.35 wt\% Ni in the neighboring kamacite. Even higher $\mathrm{Ni}$ concentrations are predicted in the taenite that is in direct contact with kamacite according to the phase diagram, so the concentrations shown here are affected by insufficient spatial resolution (Yang et al. 1996). Henbury shows wide kamacite bands $(5.76-6.23 \mathrm{wt} \% \mathrm{Ni})$ surrounded by subordinate, thin $(<100 \mu \mathrm{m})$ taenite lamellae $(10.17-11.70 \mathrm{wt} \% \mathrm{Ni})$, with large triangular or trapezoidal plessitic areas $(6.73-6.92 \mathrm{wt} \% \mathrm{Ni}$ on average). A Ni concentration profile through kamacite-taenite-plessite-taenite-kamacite is shown in Figure 1B. Canyon Diablo exhibits a homogeneous Ni distribution in its metal. Campo del Cielo and Chinga demonstrate uniform Ni distribution maps, indicating that these are fully homogeneous in terms of Ni. The range of $\mathrm{Ni}$ concentrations in the micro-drilled iron meteorite sub-samples varies from $0.04 \mathrm{wt} \%$ (Chinga) to $0.8 \mathrm{wt} \%$ (Henbury), consistent with the values reported in the literature for bulk samples.

The results of the $\mathrm{Fe}$ and $\mathrm{Ni}$ isotope ratio determination are summarized in Table 1 . The $\delta^{56 / 54} \mathrm{Fe}$ values for the 3 ordinary chondrites analyzed vary from -0.008 to $+0.015 \%$, which is in good agreement with the range reported in literature (see a compilation by Barrat et al., 2015). Unfortunately, the amount of Ni stable isotope data published in literature for chondrites is limited. For 46 ordinary chondrites, Moynier et al., reported a $\delta^{60 / 58} \mathrm{Ni}$ range of $0.49 \%$, while for 6 carbonaceous chondrites and 6 enstatite chondrites, these authors found a range of $0.27 \%$ and $0.26 \%$, respectively (Moynier et al., 2007). It should be noted that the values in that paper are reported relative to an in-house Ni standard, as NIST SRM 986 was not commercially available at that time. The recalculated $\delta^{60 / 58} \mathrm{Ni}$ values for 6 chondrites reported by Cook et al. vary from +0.05 to $+0.44 \%$ (Cook et al., 2007). In the study referred to, all these $\delta^{60 / 58} \mathrm{Ni}$ values are not reported directly due to the possibility of nonmass dependent isotope effects on ${ }^{60} \mathrm{Ni}$. As such, these values were recalculated from the $\delta^{62 / 58} \mathrm{Ni}$ values assuming the equilibrium fractionation law. Because the largest absolute non-mass dependent isotope effects reported for $\delta^{60 / 58} \mathrm{Ni}$ in iron meteorites are on the order of $0.02 \%$ (Cook et al., 2006), comparable to the precision attained in this work, these effects were assumed to be negligible. The results of $\delta^{60 / 58} \mathrm{Ni}$ for 3 ordinary chondrites, presented in this work vary from +0.149 to $+0.242 \%$. This demonstrates the non-chondritic isotopic composition of the NIST SRM $986 \mathrm{Ni}$ isotopic standard, which appears to have shifted during Ni purification by the Mond process (Tanimizu and Hirata, 2006; Steele et al., 2011). The isotopic composition of both Fe and Ni in the fusion crusts of the chondrites characterized coincides with their measured bulk compositions within analytical uncertainty, different from the work of Hezel et al. 2015, who observed that the fusion crusts of ordinary chondrites are enriched in heavy Fe isotopes. This could either result from our sampling technique, by mixing bulk meteorite material, unaffected by atmospheric entry, with sample from the fusion crust, or could alternatively represent the effect of terrestrial weathering on fusion crusts. Hezel et al. (2015) selected the samples based on their size and the presence of visibly unaltered crusts to avoid Fe isotope exchange with the terrestrial environment. In this work, only Antarctic chondrites were analyzed and the fusion crusts of the selected samples appeared relatively fresh. 
$\delta^{56 / 54} \mathrm{Fe}$ values of iron meteorites range from -0.080 to $+0.161 \%$, while $\delta^{60 / 58} \mathrm{Ni}$ shows a wider variability, between -0.054 to $+0.551 \%$. The average $\delta^{56 / 54} \mathrm{Fe}$ value for individual iron meteorites (bulk digestions and averaged sub-samples) varies from +0.015 to $+0.139 \%$, which is similar to or higher than the chondrite values reported in literature $\left(\delta^{56 / 54} \mathrm{Fe}\right.$ of -0.05 to $+0.1 \%$ for the majority of chondrites, e.g. $\delta^{56 / 54} \mathrm{Fe}$ of $+0.018 \%$ for Allende CV3; Barrat et al., 2015). Average $\delta^{60 / 58} \mathrm{Ni}$ values for individual iron meteorites (bulk digestions and averaged sub-samples) vary from +0.102 to $+0.420 \%$, within the reported range of $0.05-0.44 \%$ for bulk metal of ordinary, carbonaceous and enstatite chondrites with an average $\delta^{60 / 58} \mathrm{Ni}$ of $+0.26 \%$, as recalculated from $\delta^{62 / 58} \mathrm{Ni}$ data for 6 chondrites (Cook et al., 2007). The average $\delta^{60 / 58} \mathrm{Ni}$ results reported in the present work for Gibeon (+0.14 \%o), Henbury (+0.22\%o), and Canyon Diablo (+0.22\%o for micro-drilled sub-samples and +0.29 $\%$ for bulk analysis) correspond well with the recalculated results of Cook et al. (2007) (+0.19 \%o for Gibeon, +0.19 to $+0.22 \%$ for Henbury, and $+0.26 \%$ for Canyon Diablo).

In the metal part of pallasites, Fe is always isotopically heavier than in the corresponding olivine fraction. In contrast, $\mathrm{Ni}$ in pallasite metal is always isotopically lighter than in the corresponding olivine (Table 1). For 3 pallasites, Fukang, Esquel and CMS 04071, $\delta^{60 / 58} \mathrm{Ni}$ is negative, below the range observed for iron meteorites (Table 1). This is in agreement with the observed light Ni isotopic compositions of the metal parts of pallasites in previous work $\left(\delta^{60 / 58} \mathrm{Ni}\right.$ between -0.18 and $+0.07 \%$, recalculated based on $\delta^{62 / 58} \mathrm{Ni}$ assuming equilibriumcontrolled isotope fractionation and neglecting possible non-mass dependent input from ${ }^{60} \mathrm{Fe}$ decay; Cook et al., 2007).

For the mesosiderites analyzed in this study, the metal fractions are characterized by relatively heavier Fe isotopic compositions $\left(\delta^{56 / 54} \mathrm{Fe}\right.$ of +0.018 to $+0.120 \%$; Table 1$)$, while the corresponding silicate parts exhibit relatively light $\mathrm{Fe}$ isotopic compositions ( $\delta^{56 / 54} \mathrm{Fe}$ of -0.214 to $-0.149 \%$ ). Similar to $\mathrm{Fe}$, Ni is isotopically heavier in the metal fractions of mesosiderites $\left(\delta^{60 / 58} \mathrm{Ni}\right.$ between +0.168 and $+0.191 \%$ ) in comparison to the silicate fractions, which have relatively lighter $\mathrm{Ni}$ isotopic signatures $\left(\delta^{60 / 58} \mathrm{Ni}\right.$ between -0.149 and $+0.023 \%$; Table 1$)$.

Our results for the Fe isotope ratios in BHVO-1 and PCC-1 are in full agreement with values reported previously in literature (Telus et al., 2012, Dauphas et al., 2009). OKUM is a new reference material of powdered ultramafic komatiite that has been certified for major and trace element abundances only. As $\delta^{56 / 54} \mathrm{Fe}$ values of komatiites have previously been found to be near-chondritic ( $+0.044 \%$ on average), indicating minor fractionation during komatiite magma genesis (Dauphas et al., 2010), the -0.003 \%o result obtained for OKUM is in agreement with the conclusions of Dauphas et al. (for more information the reader is referred to figure D1 in the ESI). 


\subsection{Equilibrium isotope fractionation of $\mathrm{Fe}$ in pallasites}

Resolvable enrichment of the metal fractions of pallasites in heavy $\mathrm{Fe}$ isotopes and corresponding depletion in olivines has previously been attributed to equilibrium fractionation during core-mantle differentiation (Poitrasson et al., 2005). Figure 2 shows the Fe isotope fractionation between native metal and silicate phases in the 4 pallasites and 3 mesosiderite meteorites analyzed in this work (for the three-isotope plots of stony-iron meteorites, the reader is referred to figure B1, provided in the ESI). The literature data available for Esquel $\left(\delta^{57 / 54} \mathrm{Fe}_{\text {metal }}=+0.237 \pm 0.055 \%\right.$, $\delta^{57 / 54} \mathrm{Fe}_{\text {olivine }}=-0.084 \pm-0.051 \%$ \% $($ Poitrasson et al., 2005) validates the results obtained here (see the discussion below). Both the petrography and the distribution of trace elements suggest that the olivine and metal phases are in equilibrium (Boesenberg et al. 2012). As such, the fractionation factors $\alpha$ can be related to the equilibrium constant $K$ for the isotopic exchange between the olivine and metal:

$$
{ }^{57} F e_{\text {oliv }}+{ }^{54} F e_{\text {met }} \rightleftharpoons{ }^{54} F e_{\text {oliv }}+{ }^{57} F e_{\text {met }} ; K=\frac{{ }^{57 / 54} F e_{\text {met }}}{{ }^{57 / 54} F e_{\text {oliv }}}=\alpha=\exp (-\Delta G / k T) ;(5)
$$

Here, subscripts denote the phases between which Fe isotopes are exchanged, $G$ represents the free energy, $k$ is the Boltzmann constant and $T$ is temperature. In turn, the difference in free energy is associated with a difference in the vibrational motions of the crystal lattice due to the difference in isotope masses. More details on the theoretical background of this equation can be found in the literature (O'Neil, 1986; Hoefs, 2009; Young et al., 2015). Hence, the distribution of isotopes between phases depends on the crystal chemistry constraints, leading to an enrichment of the heavier isotopes in the phase where the chemical bonds are stiffer and the coordination number is low (Young et al., 2015). Importantly, the distribution of isotopes also depends on the equilibration temperature, which makes $\mathrm{Fe}$ isotope ratios in equilibrated phases useful as an isotopic geothermometer. Figure 3 compares the measured Fe isotope ratio data for pallasites to the isotherms calculated for the Fe isotope partitioning between metal and olivine, based on the temperature dependence of the fractionation factors $\alpha$, as determined using Mössbauer spectroscopy (Polyakov and Mineev, 2000) and nuclear resonance inelastic X-ray scattering (NRIXS) (Dauphas et al., 2012; Dauphas et al., 2014). Previous applications of Mössbauer spectroscopy for isotope geothermometry were criticized as the results obtained are sensitive to a number of complications inherent to this method (e.g., second-order Doppler shift or hyperfine field parameters caused by changes in chemical or magnetic nature), and NRIXS is preferred as a more robust method. Comparison between the Mössbauer and NRIXS calibration (Fig. 3) indicates that the latter systematically yields equilibration temperatures approximately $100{ }^{\circ} \mathrm{C}$ higher for the low-temperature region $\left(200-400{ }^{\circ} \mathrm{C}\right)$ and $200{ }^{\circ} \mathrm{C}$ higher for the high-temperature region. As the difference between the Mössbauer (Polyakov and Mineev, 2000) and NRIXS (Dauphas et al., 2012; Dauphas et al., 2014) calibrations of metal-olivine only slightly exceeds the analytical uncertainty of isotope ratio measurements, the following conclusions are independent of the calibration method applied. It should also be noted that the model used here to calculate the metal-olivine Fe isotope isotherms is simplified, because it is not accounting for a range of minor parameters that can potentially affect the measured equilibrium temperatures, such as pressure, oxygen fugacity and the effect of minor solute elements (P, S, C, Ni) on the crystallographic constraints (Shahar et al., 2008; Polyakov, 2009).

Although the absolute $\mathrm{Fe}$ isotopic composition data for the Esquel pallasite reported by Poitrasson et al. do not coincide with the data obtained in this work within the associated analytical uncertainty, both results fall on the same isotherm for an equilibration temperature of $475{ }^{\circ} \mathrm{C}$ (or $370{ }^{\circ} \mathrm{C}$ in the case of Mössbauer isotope 
thermometry calibration), demonstrating similar isotope ratio differences between the olivine and metal and initial $\mathrm{Fe}$ isotopic heterogeneity within this meteorite. The Fukang pallasite equilibrated at approximately $340{ }^{\circ} \mathrm{C}$ (or $255^{\circ} \mathrm{C}$ for Mössbauer), while the final metal-silicate isotopic equilibration in Seymchan took place at $715^{\circ} \mathrm{C}$ (550 ${ }^{\circ} \mathrm{C}$ for Mössbauer). Similarly, the reported variation of $\delta^{57 / 54} \mathrm{Fe}_{\text {metal }}$ versus $\delta^{57 / 54} \mathrm{Fe}_{\text {olvine }}$ indicates that Marjalahti, Springwater and Imilac last equilibrated at $1300{ }^{\circ} \mathrm{C}\left(1100{ }^{\circ} \mathrm{C}\right.$ for Mössbauer), $800{ }^{\circ} \mathrm{C}\left(680{ }^{\circ} \mathrm{C}\right.$ for Mössbauer) and $600{ }^{\circ} \mathrm{C}\left(500{ }^{\circ} \mathrm{C}\right.$ for Mössbauer) respectively, while CMS 04071, Eagle Station and Esquel fall on a similar trend line that represents a final metal-silicate isotopic equilibration at $475{ }^{\circ} \mathrm{C}\left(370{ }^{\circ} \mathrm{C}\right.$ for Mössbauer) (data from Zhu et al., 2002; Poitrasson et al., 2005).

Although the minor parameters that are not accounted for in this thermometric model, such as oxygen fugacity, and the effect of solutes on the crystal chemistry and the strength of the chemical bonds, can potentially affect the absolute temperature values produced in this model, these effects are expected to be nearly similar for all PMGs as a result of their formation at the relatively thin core-mantle boundary layer of the PMG parent body and their nearly similar compositions. As such, the broad range of the olivine-metal equilibration temperatures (340 to $1300{ }^{\circ} \mathrm{C}$ or 255 to $1100{ }^{\circ} \mathrm{C}$ depending on the thermometric calibration applied) is difficult to explain in terms of a formation model, in which pallasites form at a quiescent core-mantle boundary following parent body metal-silicate segregation (Boesenberg et al., 2012). On the other hand, the equilibration temperature of 1100 $1300{ }^{\circ} \mathrm{C}$ obtained for Marjalahti (Poitrasson et al. 2005) appears realistic, close to the schreibersite and $\mathrm{Fe}-\mathrm{FeS}$ eutectic temperatures (Boesenberg et al., 2012; McKibbin et al., 2016). As such, the equilibrium isotope fractionation observed here and in previous works could be attributed to a later-stage, presently unidentified (possibly incomplete) re-equilibration event, different from the core-mantle segregation. This process clearly disturbed the $\mathrm{Fe}$ isotopic composition of the initially equilibrated metal and olivine. Possibly, a collisional event and large scale melting was followed by the removal of the solidified core, after which the pallasites (incompletely) equilibrated at various temperatures, buried at different depths in a secondary-accreted parent body (Yang et al., 2010, Ruzicka 2014). Alternatively, aqueous alteration may be a process that re-distributed Fe isotopes between olivine and accessory minerals in small cracks and veinlets (Telus et al., 2016).

Alternatively, a potential explanation for the low equilibration temperature measured for Fukang could be the sensitivity of the thermometric method to sampling, as the measured isotopic composition of the metal fraction might not be fully representative of its bulk-metal isotopic composition (e.g., by sampling a larger fraction of isotopically heavier taenite, which shifts the equilibration temperature obtained to the colder side of the plot). But even if the lowest temperature determined for Fukang is disqualified as unrealistically low and is considered to result from oversampling of isotopically heavy taenite, unrepresentative sampling cannot account for another "cold" PMG - Esquel, for which the Fe isotope temperature coincides with that reported in previous publications (Poitrasson et al. 2005) and is confirmed by the analysis of other pallasites (CMS 04071, Eagle Station).

The equilibration temperatures of $475{ }^{\circ} \mathrm{C}$ have previously been noted to be unrealistically low for pallasites (Poitrasson et al. 2005), because it is unlikely that $\mathrm{cm}$-sized olivine crystals reached full equilibration with their surrounding metal in terms of Fe isotopes. Based on the diffusion data of Jaoul et al., 1995, the full equilibration at such temperatures has been estimated to take an unrealistic amount of time, on the order of several Gyr. Such low temperatures for some pallasites were explained by incomplete equilibration and disturbance by diffusion effects. However, more refined studies of diffusion demonstrate that diffusion 
coefficients highly depend on multiple parameters, such as crystallographic orientation, making estimations of equilibration time highly imprecise (Dohmen et al., 2007; Dohmen and Chakraborty, 2007). Additionally, previously measured electron microprobe (EMPA) concentration profiles have shown that olivines in the Esquel pallasite are compositionally uniform, with only slight $<0.5 \mathrm{~mol} \%$ zoning of fayalite $(\mathrm{Fa}=\mathrm{Fe} /(\mathrm{Mg}+\mathrm{Fe})$; Miyamoto, 1997). Whether this abundance gradient is correlated with isotopic zoning is a task for more detailed in situ work, but it seems unrealistic that such limited concentration profile is accompanied by a shift in the $\delta^{57 / 54} \mathrm{Fe}$ value of olivine by approximately $0.2 \%$, needed to obtain more realistic equilibration temperatures (e.g. see Oeser et al., 2015 or Sio et al., 2013). Besides faster cooling, the higher abundance of olivine to metal in Imilac, Eagle Station and Esquel compared to Marjalahti and Springwater has been suggested to be another factor limiting isotopic re-equilibration, because of the larger average distance the $\mathrm{Fe}$ atoms have to diffuse into olivine to equilibrate with the adjacent metal (Zhu et al., 2002; Poitrasson et al., 2005).

\subsection{Isotope fractionation of $\mathrm{Fe}$ in IIIAB and PMG meteorites as a result of fractional crystallization} of asteroidal cores

IIIAB iron meteorites are known to form a fractional crystallization sequence, with decreasing concentrations of Ir (and particular other elements) as the crystallization proceeds, and the compositions of PMG (e.g., Au-As trends) are broadly compatible with late-stage crystallization of a core with IIIAB-like composition, suggesting a possible genetic relationship (although additional explanations are needed to explain $\mathrm{Ga}$ and $\mathrm{Ge}$ trends) (Scott, 1977b; Scott, 1977c; Wasson and Choi, 2003). There are currently several arguments pro and contra- a shared origin of these meteorites. Potential evidence for a common origin of IIIAB and pallasite metal is based on similar oxygen three-isotope compositions for pallasites and chromite inclusions in particular IIIAB irons, although this is not the case for Cape York IIIAB, having significantly distinct oxigen isotope signatures (Franchi et al., 2013). Similarly, the Mo isotopic signatures for the metal phases (Dauphas et al., 2002) and the Hf-W short-lived isotopic decay system (Quitté et al., 2005) suggest a common origin for IIIAB and PMG meteorites. On the other hand, the Re-Os isotope chronometer has produced a whole-rock pallasite isochron distinct from those of iron meteorites, indicating that iron meteorites underwent fractional crystallization processes $\sim 60$ Myr later than pallasite metals (Chen et al., 2002). Metallographic studies of metal phases imply that below $975 \mathrm{~K}$, IIIAB irons experienced faster cooling rates than pallasites and that different pallasites cooled at disparate rates, probably because this cooling took place at a different depth in the parent body (Yang et al., 2010; Goldstein et al., 2014). Although the range of non-mass-dependent $\mathrm{W}$ isotopic signatures within the IIIAB iron group is narrower than for whole iron meteorites (Markowski et al., 2006), which makes the genetic link between pallasites and IIIAB iron meteorites elusive, mass-dependent $\mathrm{W}$ isotopic compositions of IIIAB and pallasites fall on the same mass-dependent isotope fractionation line (Fukami et al., 2010). Negative correlation of $\mathrm{W}$ isotope fractionation to Ir suggests that $\mathrm{W}$ stable isotopes in IIIAB and pallasites fractionated during fractional crystallization of liquid metal (Fukami et al., 2010).

Figure 4 plots the Ni and $\mathrm{Fe}$ isotope compositions for PMG and IIIAB against their Ir concentrations. Figure $4 \mathrm{~b}$ indicates a trend between the $\mathrm{Fe}$ isotope ratio and the Ir content, as a result of fractional crystallization of the parent body core(s). The trends for PMG and IIIAB iron meteorites are similar, with heavier Fe isotope ratios as the Ir concentration decreases. As $\delta^{57 / 54} \mathrm{Fe}$ in PMG and IIIAB group meteorites display a nearly identical trend versus $\mathrm{Ir}$, this might indicate similar crystallization processes in their parent bodies, and could confirm a shared parent body for PMGs and IIIAB iron meteorites. In contrast to IIIAB and metal fractions of PMGs, no 
trend was found for IIAB iron meteorites in a plot of $\delta^{57 / 54} \mathrm{Fe}$ versus Ir content (ESI, figure B3). The fact that IIAB irons do not show a similar trend is indeed enigmatic, and we have to invoke possible effects of initial concentrations of minor elements or the size of the parent bodies and corresponding cooling rates or later disturbances of the Fe isotope signatures. ESI contains more detailed modeling of Fe isotope fractionation during metal differentiation.

\subsection{Isotope fractionation of $\mathrm{Ni}$ in pallasites: equilibrium or kinetic in nature?}

The Ni isotopic composition of stony-iron meteorites is presented in Figure 2. Different to Fe, the light Ni isotopic composition of pallasite metals in comparison to iron meteorites argues against a derivation of pallasites from the IIIAB iron meteorite parent body that are reported to have heavier Ni stable isotope signatures (this study, Cook et al., 2007). This is in contrast to the trend observed for Ir concentrations versus Fe isotope ratios.

Fractional crystallization of a molten metal core in the PMG-IIIAB parent body cannot explain the light $\mathrm{Ni}$ isotopic signatures measured. Any trends in a plot of ${ }^{60} \mathrm{Ni} /{ }^{58} \mathrm{Ni}$ ratios versus $\mathrm{Ir}$ concentrations remain elusive, which demonstrates the limited preservation of fractional crystallization effects on the Ni isotopic compositions of the PMG metal fraction (Figure 4). Also, scenarios admixing Ni from external sources during PMG formation appear unlikely, as $\mathrm{Ni}$ is a major element and unrealistically high amounts are needed to account for a shift in the metal $\mathrm{Ni}$ isotopic signatures to lighter values. The light isotopic composition in the metal phase of the PMG can also not be explained by condensation from a vapor phase in a Rayleigh-like process, for instance as a result of planetary collision, because in that case, a matched enrichment in light isotopes of Fe would be found, which is not the case.

Several possible scenarios can be invoked to explain the light isotopic composition of $\mathrm{Ni}$ in the metal fractions of pallasites compared to a heavier Ni isotopic signature in the adjacent olivine:

(i) The sampling of metal might not be representative for the bulk $\mathrm{Ni}$ isotopic composition. Light $\mathrm{Ni}$ isotopic compositions can be caused by oversampling of particular metal phases, e.g., taenite or swathing kamacite, which is not reflected in the Fe isotopes due to the higher overall abundance of Fe and smaller isotopic differences between kamacite and taenite. However, the light stable isotopic signatures of Ni measured in metals are in full agreement with those previously reported for the metal fractions of 3 other pallasites (Cook et al., 2007).

(ii) The heavy Ni stable isotopic composition of olivine and the comparatively lower values for the corresponding metal could result from equilibrium isotope fractionation during core-mantle segregation. In this case, the reversed character of metal-olivine fractionation factors for $\mathrm{Ni}$ and $\mathrm{Fe}$ is striking, while these 2 elements possess nearly similar physical properties, such as volatilities and ionic radii. One characteristic, which differentiates $\mathrm{Ni}$ from $\mathrm{Fe}$, is its inability to take on a 3+ oxidation state. As such, a redox mechanism that reverses the fractionation factor of $\mathrm{Fe}$ with respect to that of $\mathrm{Ni}$ might hypothetically be involved, for instance if charge-balancing is needed for Fe substitution of trivalent ions in olivine. Different to Fe, the theoretical silicatemetal fractionation factors for $\mathrm{Ni}$ are scarcely studied, but experimental work did indicate an enrichment of heavy $\mathrm{Ni}$ isotopes in metal relative to talc at temperatures of $500^{\circ} \mathrm{C}$ to $950^{\circ} \mathrm{C}$ and pressures of $0.8-1.3 \mathrm{GPa}$ (Lazar et al., 2012). To clarify the equilibrium fractionation constraints, the crystal chemistry of Ni in olivine should be studied in more detail, because the bonding environment is a factor affecting the isotope fractionation strongly. Different to $\mathrm{Fe}, \mathrm{Ni}$ is a trace element in olivine, and can either substitute $\mathrm{Fe}$ in the crystal lattice of olivine, be compensated for by vacancies, or be concentrated in inclusions. Figure 5 presents a 2D LA-ICP-MS 
distribution image of $\mathrm{Ni}$ in olivine of Seymchan, representative for all PMGs evaluated in this study. The veins with elevated concentrations of $\mathrm{P}$ and $\mathrm{Ni}$ probably have a shock origin (Desrousseaux et al., 1997). The concentration of $\mathrm{Ni}$ in bulk olivine is of the order of $10 \mu \mathrm{g} \mathrm{g}^{-1}$, while Ni concentrations of up to several thousands $\mu \mathrm{g} \mathrm{g}^{-1}$ are found in the veinlets. This indicates that a significant part of the $\mathrm{Ni}$ is contained in the veins of the bulk silicate. If the process that formed these features also led to $\mathrm{Ni}$ exchange between metal, olivine and veins, after the initial olivine/metal $\mathrm{Ni}$ isotopic equilibration, this could have led to a significant shift in the $\mathrm{Ni}$ isotopic composition of the bulk silicate (olivine + vein).

(iii) Light Ni stable isotopic signatures of metal and heavy signatures of adjacent olivine could result from disequilibration of the initial isotopic signatures. The initial isotopic composition of metal and olivine can be affected by a later thermal event, such as impact or internal heating, and disturbed by kinetic isotope fractionation during solid-state diffusion of $\mathrm{Ni}$ between metal/olivine/veins. However, the fact that $\mathrm{Ni}$ in silicate is consistently isotopically heavier than that in the adjacent metal indicates the direction of possible Ni diffusion from olivine into the metal as light isotopes diffuse faster, which is opposite to the concentration gradient. However, taking into account that $\mathrm{Ni}$ is a siderophile element, the chemical potential gradient can easily be opposite to the concentration gradient in the case of the metal-olivine boundary, which would allow for selfdiffusion of $\mathrm{Ni}$ isotopes. Previous work has presented concentration gradients of doubly charged ions in pallasitic olivines due to solid-state diffusion (Tomiyama and Huss, 2006; McKibbin et al., 2013). These profiles are suggested to reflect re-equilibration of olivine and metal, mixed as a result of an impact, that is followed by fast cooling. However, taking mass-balance considerations into account, it is unlikely that diffusion of $\mathrm{Ni}$ isotopes from olivine into the metal could explain light $\mathrm{Ni}$ isotope signatures of the metal phase, because of its several orders of magnitude lower content in olivine compared to metal.

As the mechanism of the $\mathrm{Ni}$ distribution between metal and olivine remains unclear, more spatially resolved in situ isotopic analyses of closely associated metal, olivine and possibly $\mathrm{Ni}$ contained in veins, are needed to shed light onto the $\mathrm{Ni}$ isotope fractionation mechanism.

\subsection{Origin of metal and silicate in mesosiderites}

Mesosiderites have enigmatic formation histories, with most models suggesting that they formed by mixing of material from two different parent bodies. Silicate clasts of mesosiderites indicate their origin from the evolved crust of a differentiated asteroid (Stewart et al., 1994, Greenwood et al., 2015, Haack et al., 2003), potentially linking it to the crust of asteroid 4 Vesta (McSween et al., 2011). Mesosiderites have a restricted range of metal compositions, suggesting that the metal did not undergo fractional crystallization and that the metal was molten when mixed with the colder silicates (Hassanzadeh et al., 1990). The compositional range of the metallic portions of mesosiderites suggests that mesosiderite metal may have crystallized from a core with an original melt composition similar to IIIAB and H-group chondrite metal (Wasson et al., 1974). Differences between slow metallographic cooling rates at $500{ }^{\circ} \mathrm{C}$ (Goldstein et al., 2014) and fast cooling of pyroxene clasts at peak metamorphic temperatures above $800{ }^{\circ} \mathrm{C}$ (Ruzicka et al., 1994; Schwandt et al., 1998), probably due to equilibration of mixed hot and cold fragments, suggest a change of cooling regime when mesosiderites were buried deep in a re-accreted parent body (Bogard and Garrison, 1998).

The ${ }^{56 / 54} \mathrm{Fe}$ isotopic compositions of the metal fractions of 3 Antarctic mesosiderites vary between +0.018 and $+0.120 \%$ (Figure 2, Table 1), which is close to the values for chondritic (this study; Barrat et al., 2015) and iron meteorites (this study; Poitrasson et al., 2005; Williams et al., 2006) (Figure 2, Table 1). The Ni isotopic 
composition of the metal fractions is comparable to or slightly lighter than that of chondrites and iron meteorites (Fig. 2). The Fe and Ni stable isotope signatures in the metal fractions of mesosiderites are within the range of chondrites or iron meteorites, which is in agreement with the trace element compositions, suggesting that the metal in mesosiderites shares an origin with chondrites or asteroidal metal cores (Wasson et al., 1974).

The isotopic signatures of both $\mathrm{Fe}$ and $\mathrm{Ni}$ in the silicate portions of mesosiderites are relatively light (Figure 2, Table 1). These signatures can either be derived from the basaltic regolith of the differentiated asteroid, from which the silicate fraction of mesosiderites originates, or be acquired during the evolution of the mesosiderites. As stable Ni isotope data for asteroidal crusts in literature are rare, this is hard to evaluate in the case of Ni. However, the negative Fe isotopic signatures in the silicate portions of mesosiderites may indicate that these result from late-stage evolution and isotope fractionation, because all the terrestrial and extraterrestrial crust silicates reported to date have either chondritic or heavy Fe isotopic compositions (e.g., Barrat et al., 2015). Therefore, the light $\mathrm{Fe}$ and associated light $\mathrm{Ni}$ isotopic signatures of mesosiderites are more likely to result from diffusion of light $\mathrm{Fe}$ and $\mathrm{Ni}$ isotopes out of hot metal into silicates that initially showed chondritic or heavier isotope ratios following the formational mixing event. The $\mathrm{Fe}$ and $\mathrm{Ni}$ isotopic signatures of the metal phases might not have been affected significantly by this diffusion because of the higher overall concentrations of $\mathrm{Fe}$ and $\mathrm{Ni}$, and thus remained unchanged.

\subsection{Kinetic isotope fractionation of $\mathrm{Fe}$ and $\mathrm{Ni}$ in iron meteorites due to sub-solidus diffusion}

The Toluca IAB iron meteorite was extensively studied for Fe-Ni isotope fractionation as a result of Widmanstätten pattern growth (Poitrasson et al., 2005; Dauphas, 2007; Cook et al., 2007). Due to slow cooling of meteorite parent bodies, taenite changes into this structure of kamacite/taenite. If kamacite and taenite are in full equilibrium, isotope fractionation is controlled by the crystal chemistry constraints and temperature in a manner similar to that described for pallasites. At the same time, kamacite grows via sub-solidus diffusion of Fe and $\mathrm{Ni}$ atoms, and as light isotopes tend to diffuse faster than heavy ones, a factor of kinetic control is added to the isotope fractionation mechanism. For a detailed description of the isotope fractionation mechanism during disequilibrium crystal-growth phenomena, also for the particular case of Widmanstätten pattern growth, the reader is referred to earlier publications (Dauphas, 2007; Watson and Müller, 2009; Mueller et al., 2014).

However, Toluca belongs to the IAB complex silicate-bearing iron meteorite group, which has experienced impact processing during its formation history (Goldstein et al., 2009; Ruzicka, 2014). To expand the pool of data available, two magmatic iron meteorites of different groups (Henbury IIIAB and Gibeon IVA) with distinct cooling histories were also studied in this work. Four to 10 drillings on each etched sample surface were performed to represent the spread of isotope ratios contained in the inhomogeneous iron meteorite body. Because the drilling is done with a finite spot size (300 $\mu \mathrm{m}$ diameter) and because the phase composition cannot be controlled with the depth of the drilling $(400 \mu \mathrm{m})$, all of the micro-drilled samples represent mixtures of kamacite and taenite in different proportions (including the plessitic fine mixture of both minerals). The highest and the lowest isotope ratios measured in this way can be assumed to approximate the lowest and highest estimates of isotopic compositions in the center of the kamacite and taenite phases, respectively. However, given the large number of local measurements, a fractionation line can be constructed with higher precision. Figure 6 provides three-isotope plots based on data from spatially resolved analyses of iron meteorites for their $\mathrm{Fe}$ and $\mathrm{Ni}$ isotopic compositions. The theoretical equilibrium $\left(\beta_{e q}\right)$ and kinetic $\left(\beta_{\text {kin }}\right)$ fractionation factors can be calculated (Wombacher and Rehkamper, 2003; Young et al. 2002). The experimental fractionation factor, extracted from 
$\delta^{62 / 58} \mathrm{Ni}-\delta^{60 / 58} \mathrm{Ni}$ data $\left(\beta_{\exp }=1.981 \pm 0.039\right)$ corresponds best to kinetically controlled isotope fractionation. This confirms that the contribution of sub-solidus diffusion prevails over equilibrium isotope fractionation control (Dauphas, 2007). The fractionation factors $\beta_{\text {exp }}$ for Fe and the other Ni isotopes show less precision as a result of the limited amount of $\mathrm{Fe}$ isotope fractionation or the lower abundance of the corresponding $\mathrm{Ni}$ isotopes, preventing their use in this context. It is interesting to note that the average of the data obtained for 7 spatially resolved analyses of the Henbury IIIAB iron meteorite measured in this study $\left(\delta^{60 / 58} \mathrm{Ni}=+0.22 \%\right)$ corresponds well to previously reported bulk analyses data, varying between $+0.22 \%$ and $+0.19 \%$ (Cook et al., 2007).

Magmatic iron meteorite groups exhibit element trends compatible with fractional crystallization, initiated by the cooling of molten asteroid cores. The silicate-bearing groups (IIE group and the IAB complex, which includes the former IIICD) are believed to have formed through impact of melted bodies and as such, experienced a different crystallization and cooling history (Ruzicka, 2014). The range of stable isotope signatures of $\mathrm{Ni}$ is wider than that of $\mathrm{Fe}$ in laterally resolved samples due to the effect of sub-solidus diffusion and its lower concentration relative to Fe. However, for the silicate-bearing IAB-MG iron meteorite Canyon Diablo, and especially for Campo del Cielo, this effect is less pronounced. This could result from impact processing, when the isotope ratios of both $\mathrm{Fe}$ and $\mathrm{Ni}$ in silicate-bearing irons were first shifted to heavier values as a result of evaporation following impact (the 50\% condensation temperatures in a Solar System composition gas are 1353 and $1334 \mathrm{~K}$ for $\mathrm{Ni}$ and Fe, respectively; Lodders, 2003). Subsequently, the Fe and Ni isotopes were fractionated between kamacite/taenite as a result of sub-solidus diffusion, although to a lesser degree than experienced by the magmatic groups, as a result of faster cooling rates (Goldstein et al., 2014). The cooling rate will affect the degree of kinetic fractionation because of its effect on diffusive closure and because it will control the growth rate of the exsolution features. However, it is impossible to determine the timing of the impact from the Fe-Ni isotopic signatures. The effect of impact processing on the isotopic composition of Ge has previously been described for silicate-bearing iron meteorites, which show lower and more variable Ge isotopic compositions than do magmatic irons (Luais, 2007).

The Udei Station meteorite consists of a mixture of metal and silicates. However, it is classified as IABungrouped according to the Meteoritical Bulletin, based on the composition of its metal fraction (Wasson, 1970a), the petrographic characteristics of the silicates (Mason, 1967), and the higher metallographic cooling rates compared to mesosiderites (Powell, 1969). The Fe and Ni isotopic signatures in the metal phase of Udei Station are close to those of other iron meteorites, but negative in the silicate fraction. These negative signatures are unlikely to derive from the initial parent material of the silicates, as there is strong evidence for a chondritic origin (Choi et al., 1995; Wasson and Kallemeyn, 2002). Consequently, the isotopic signatures of the Udei Station silicate might result from $\mathrm{Fe}$ and Ni diffusion out of the metal phase of this meteorite, similar to what has been observed for mesosiderites. On the other hand, recent work suggests that the silicates in Udei Station experienced low degrees (3-10\%) of partial melting, which could potentially have fractionated the $\mathrm{Fe}$ and $\mathrm{Ni}$ isotopes (Ruzicka and Hutson, 2010). 
This study contributes high-precision $\mathrm{Fe}$ and $\mathrm{Ni}$ isotope ratio data for bulk magmatic and silicate-bearing iron, pallasite, and mesosiderite meteorites obtained via MC-ICP-MS to the existing literature. Importantly, cooccurring silicate and metal portions for various stony iron meteorites were compared in terms of their Fe and $\mathrm{Ni}$ isotopic compositions. For this purpose, relatively large sample aliquots (generally > $100 \mathrm{mg}$ ) guaranteed representative sampling considering the isotopic heterogeneity at the micrometer to millimeter scale that generally reflects sub-solidus diffusion. In the case of magmatic and silicate-bearing iron meteorites, microdrilling using a $300 \mu \mathrm{m}$ diameter drill bit also allowed laterally resolved isotopic analysis of Fe and Ni. Unlike chondrites, for which the $\mathrm{Fe}$ and $\mathrm{Ni}$ isotope signatures result from nebular processes and the isotopic range is limited to $\sim 0.2 \%$ (Moynier et al, 2007; Craddock and Dauphas 2011), metal-rich achondritic materials such as iron and stony-iron meteorites typically exhibit much wider ranges of up to $0.6 \%$ for $\delta^{56 / 54} \mathrm{Fe}$ and $\sim 2 \%$ for $\delta^{60 / 58} \mathrm{Ni}$ between the metal and silicate phase in stony irons. By comparing the magnitude and sign of the $\mathrm{Fe}$ and $\mathrm{Ni}$ isotope stable isotope shifts between meteorite groups and fractions, it becomes clear that the stable isotope signatures of these achondrites result from unique combinations of distinct planetary processes, such as metal segregation and fractional crystallization during core formation, primary equilibration between planetary reservoirs of pristine parent bodies, evaporation during collisional disruption, diffusive re-equilibration during re-accretion on secondary parent bodies, and subsequent diffusion during prolonged cooling histories. As such, stable isotope signatures can also be used to highlight possible genetic relations (e.g., between pallasite metal and IIIAB irons).

The stable isotope fractionation of $\mathrm{Fe}$ and $\mathrm{Ni}$ related to sub-solidus diffusion exhibits a narrower range for silicate-bearing than for magmatic iron meteorites. This is interpreted to reflect the slower cooling rates experienced by the latter. Additionally, impact volatilization may also have played a role in the $\mathrm{Ni}$ and $\mathrm{Fe}$ isotopic signatures of silicate-bearing iron meteorites. In contrast, Fe isotopes of the PMG and in the IIIAB parent bodies' metal core(s) fractionated in the process of fractional crystallization, as revealed by the Ir concentration $-\delta^{56 / 54} \mathrm{Fe}$ trend. No such Fe fractional crystallization trend is found for the IIAB iron meteorite group, the only other iron meteorite group for which enough high-precision $\mathrm{Fe}$ isotope data exists. Unlike $\mathrm{Fe}, \mathrm{Ni}$ isotopes do not indicate fractionation coupled to the fractional crystallization of the parent body core.

In the case of mesosiderites, light $\mathrm{Ni}$ and $\mathrm{Fe}$ isotopic signatures were observed in the silicate portions analyzed relative to the heavier, near-chondritic signatures of the corresponding metal phases. These compositions likely result from diffusion of $\mathrm{Ni}$ and $\mathrm{Fe}$ from the metal into the silicate portion of these meteorites, following the mixing between an impactor and the original parent body.

The metal and olivine portions of pallasites show resolvable Fe isotope fractionation. However, if the Fe isotopes did originally achieve equilibrium, the extension of the wide range of derived equilibration temperatures $\left(255-340^{\circ} \mathrm{C}\right.$ to $\left.1100-1300^{\circ} \mathrm{C}\right)$ for different groups of pallasites suggests that $\mathrm{Fe}$ isotope fractionation is not the result of a core-mantle segregation event, but rather represents later re-equilibration at different depths in a secondary parent body. Nickel shows an isotope fractionation factor between metal and olivine in pallasites that is reversed compared to that of $\mathrm{Fe}$, with lighter isotopic compositions for metal $\left(\delta^{60 / 58} \mathrm{Ni}\right.$ from -0.81 to +0.455 $\%$ ) and heavier compositions for the corresponding olivine $\left(\delta^{60 / 58} \mathrm{Ni}\right.$ from +0.554 to $+1.221 \%$ ). This result is unlikely to derive from equilibrium isotope fractionation during core-mantle segregation, as Fe and Ni generally exhibit similar chemical properties. Similarly, a control by kinetic isotope fractionation linked to Ni diffusion out 
1 of the olivine into the metal phase would not explain the light $\mathrm{Ni}$ isotopic signatures in pallasite metal. Because 2 the crystal chemistry constraints on Ni remain unclear and large portions of silicate Ni were found to reside in 3 inclusions, additional in situ analyses are needed to shed light on the actual mechanism of $\mathrm{Ni}$ isotope 4 fractionation. 
This research project has been funded by the Planet Topers, Interuniversity Attraction Poles Program initiated by the Belgian Science Policy Office (BELSPO). Steven Goderis and Marta Costas Rodríguez are postdoctoral fellows of the Research Foundation - Flanders (FWO). The support of the Hercules Foundation is acknowledged. US Antarctic meteorite samples are recovered by the Antarctic Search for Meteorites (ANSMET) program, which has been funded by NSF and NASA, and characterized and curated by the Department of Mineral Sciences of the Smithsonian Institution and Astromaterials Curation Office at NASA Johnson Space Center. The collection of iron meteorites from east Russia and northern Kazakhstan was kindly donated by Felix P. Lesnov (IGM SB RAS). The ordinary chondrites, provided by the RBINS, are part of the Antarctic meteorite collection that was recovered through the SAMBA project, also founded by BELSPO, in collaboration with the Japanese National Institute of Polar Research (NIRP). Frank Vanhaecke acknowledges Teledyne Cetac Technologies for financial support of his LA-ICP-MS research projects. Authors acknowledge the highly constructive comments supplied by two anonymous reviewers, Thomas Mueller (University of Leeds), and by the editor Nicolas Dauphas (University of Chicago). Stef Vansteenberge and Christina Makarona (VUB) are kindly acknowledged for their technical help with the MicroMill and XRF units. The authors appreciate valuable discussions with Seann McKibbin and Lidia Pittarello (VUB). 
Alexander C. M. O. and Wang J. (2001) Iron isotopes in chondrules: Implications for the role of evaporation during chondrule formation. Meteorit. Planet. Sci. 36, 419-428.

Barrat J. A., Rouxel O., Wang K., Moynier F., Yamaguchi A., Bischoff A. and Langlade J. (2015) Early stages of core segregation recorded by Fe isotopes in an asteroidal mantle. Earth Planet. Sci. Lett. 419, 93-100.

Baxter D. C., Rodushkin I., Engström E. and Malinovsky D. (2006) Revised exponential model for mass bias correction using an internal standard for isotope abundance ratio measurements by multi-collector inductively coupled plasma mass spectrometry. J. Anal. At. Spectrom. 21, 427-430.

Boesenberg J. S., Delaney J. S. and Hewins R. H. (2012) A petrological and chemical reexamination of Main Group pallasite formation. Geochim. Cosmochim. Acta 89, 134-158.

Bogard D. D. and Garrison D. H. (1998) ${ }^{39} \mathrm{Ar}-{ }^{40} \mathrm{Ar}$ ages and thermal history of mesosiderites. Geochim. Cosmochim. Acta 62, 1459-1468.

Cameron V. and Vance D. (2014) Heavy nickel isotope compositions in rivers and the oceans. Geochim. Cosmochim. Acta 128, 195-211.

Cameron V., Vance D., Archer C. and House C. H. (2009) A biomarker based on the stable isotopes of nickel. Proc. Natl. Acad. Sci. U. S. A. 106, 10944-10948.

Chabot N. L. and Haack H. (2006) Evolution of Asteroidal Cores. In Meteorites and the Early Solar System II University of Arizona Press. pp. 747-771.

Chen J. H., Papanastassiou D. and Wasserburg G. (2002) Re-Os and Pd-Ag systematics in Group IIIAB irons and in pallasites. Geochim. Cosmochim. Acta 66, 3793-3810.

Chernonozhkin S. M., Goderis S., Bauters S., Vekemans B., Vincze L., Claeys P. and Vanhaecke F. (2014) Evaluation of pneumatic nebulization and ns-laser ablation ICP-MS for bulk elemental analysis and 2dimensional element mapping of iron meteorites. J. Anal. At. Spectrom. 29, 1001-1016.

Chernonozhkin S. M., Goderis S., Lobo L., Claeys P. and Vanhaecke F. (2015) Development of an isolation procedure and MC-ICP-MS measurement protocol for the study of stable isotope ratio variations of nickel. J. Anal. At. Spectrom. 30, 1518-1530.

Choi B., Ouyang X. and Wasson J. T. (1995) Classification and origin of IAB and IIICD iron meteorites. Geochim. Cosmochim. Acta 59, 593-612.

Cook D. L., Wadhwa M., Clayton R. N., Dauphas N., Janney P. E. and Davis A. M. (2007) Mass-dependent fractionation of nickel isotopes in meteoritic metal. Meteorit. Planet. Sci. 42, 2067-2077.

Cook D. L., Wadhwa M., Janney P. E., Dauphas N., Clayton R. N. and Davis A. M. (2006) High precision measurements of non-mass-dependent effects in nickel isotopes in meteoritic metal via multicollector ICPMS. Anal. Chem. 78, 8477-8484.

Craddock P. R. and Dauphas N. (2011) Iron isotopic compositions of geological reference materials and chondrites. Geostand. Geoanalytical Res. 35, 101-123. 
Craddock P. R., Warren J. M. and Dauphas N. (2013) Abyssal peridotites reveal the near-chondritic Fe isotopic composition of the Earth. Earth Planet. Sci. Lett. 365, 63-76.

Danielson L. R., Righter K. and Humayun M. (2009) Trace element chemistry of Cumulus Ridge 04071 pallasite with implications for main group pallasites. Meteorit. Planet. Sci. 44, 1019-1032.

Dauphas N. (2007) Diffusion-driven kinetic isotope effect of Fe and Ni during formation of the Widmanstätten pattern. Meteorit. Planet. Sci. 42, 1597-1613.

Dauphas N., Cates N. L., Mojzsis S. J. and Busigny V. (2007) Identification of chemical sedimentary protoliths using iron isotopes in the $>3750$ Ma Nuvvuagittuq supracrustal belt, Canada. Earth Planet. Sci. Lett. 254, $358-376$.

\section{Dauphas N., Cook D. L., Sacarabany A., Fröhlich C., Davis A. M., Wadhwa M., Pourmand A., Rauscher T. and} Gallino R. (2008) Iron 60 evidence for early injection and efficient mixing of stellar debris in the protosolar nebula. The Astrophysical Journal, 686, 560-569.

Dauphas N., Craddock P. R., Asimow P. D., Bennett V. C., Nutman A. P. and Ohnenstetter D. (2009) Iron isotopes may reveal the redox conditions of mantle melting from Archean to Present. Earth Planet. Sci. Lett. 288, 255-267.

Dauphas N., Janney P. E., Mendybaev R. A., Wadhwa M., Richter F. M., Davis A. M., van Zuilen M., Hines R. and Foley C. N. (2004) Chromatographic separation and multicollection-ICPMS analysis of iron. Investigating mass-dependent and -independent isotope effects. Anal. Chem. 76, 5855-63.

Dauphas N., Marty B. and Reisberg L. (2002) Molybdenum evidence for inherited planetary scale isotope heterogeneity of the protosolar nebula. Astrophys. J. 565, 640-644.

Dauphas N., Roskosz M., Alp E. E., Golden D. C., Sio C. K., Tissot F. L. H., Hu M. Y., Zhao J., Gao L. and Morris R. V. (2012) A general moment NRIXS approach to the determination of equilibrium Fe isotopic fractionation factors: Application to goethite and jarosite. Geochim. Cosmochim. Acta 94, 254-275.

Dauphas N., Roskosz M., Alp E. E., Neuville D. R., Hu M. Y., Sio C. K., Tissot F. L. H., Zhao J., Tissandier L., Médard E. and Cordier C. (2014) Magma redox and structural controls on iron isotope variations in Earth's mantle and crust. Earth Planet. Sci. Lett. 398, 127-140.

Dauphas N., Teng F.-Z. and Arndt N. T. (2010) Magnesium and iron isotopes in 2.7 Ga Alexo komatiites: Mantle signatures, no evidence for Soret diffusion, and identification of diffusive transport in zoned olivine. Geochim. Cosmochim. Acta 74, 3274-3291.

Deng T.-H.-B., Cloquet C., Tang Y.-T., Sterckeman T., Echevarria G., Estrade N., Morel J.-L. and Qiu R.-L. (2014) Nickel and zinc isotope fractionation in hyperaccumulating and nonaccumulating plants. Environ. Sci. Technol. 48, 11926-11933.

Desrousseaux A., Doukhan J. C., Leroux H. and Van Duysen J. C. (1997) An analytical electron microscope investigation of some pallasites. Phys. Earth Planet. Inter. 103, 101-115. 
Dohmen R., Becker H.-W. and Chakraborty S. (2007) Fe-Mg diffusion in olivine I: experimental determination between 700 and $1,200^{\circ} \mathrm{C}$ as a function of composition, crystal orientation and oxygen fugacity. Phys. Chem. Miner. 34, 389-407.

Dohmen R. and Chakraborty S. (2007) Fe-Mg diffusion in olivine II: point defect chemistry, change of diffusion mechanisms and a model for calculation of diffusion coefficients in natural olivine. Phys. Chem. Miner. 34, 409-430.

Estrade N., Cloquet C., Echevarria G., Sterckeman T., Deng T., Tang Y. and Morel J.-L. (2015) Weathering and vegetation controls on nickel isotope fractionation in surface ultramafic environments (Albania). Earth Planet. Sci. Lett. 423, 24-35.

Fehr M. A., Andersson P. S., Hålenius U. and Mörth C.-M. (2008) Iron isotope variations in Holocene sediments of the Gotland Deep, Baltic Sea. Geochim. Cosmochim. Acta 72, 807-826.

Franchi I. A., Greenwood R. C. and Scott E. R. D. (2013) The IIIAB-pallasite relationship revisited the oxigen isotope perspective. In 76th Annual Meteoritical Society Meeting p. 5326.

Fukami Y., Kimura J., Irisawa K., Yokoyama T. and Hirata T. (2010) Mass-dependent fractionation of tungsten isotopes in IIIAB iron meteorites. In Lunar Planet. Sci. XLI. The Woodlands, Texas. \#1649 (abstr.).

Gall L., Williams H. M., Siebert C., Halliday A. N., Herrington R. J. and Hein J. R. (2013) Nickel isotopic compositions of ferromanganese crusts and the constancy of deep ocean inputs and continental weathering effects over the Cenozoic. Earth Planet. Sci. Lett. 375, 148-155.

Goldstein J. I., Scott E. R. D. and Chabot N. L. (2009) Iron meteorites: Crystallization, thermal history, parent bodies, and origin. Chemie der Erde - Geochemistry 69, 293-325.

Goldstein J. I., Yang J. and Scott E. R. D. (2014) Determining cooling rates of iron and stony-iron meteorites from measurements of $\mathrm{Ni}$ and $\mathrm{Co}$ at kamacite-taenite interfaces. Geochim. Cosmochim. Acta 140, 297320.

Greenwood R. C., Barrat J.-A., Scott E. R. D., Haack H., Buchanan P. C., Franchi I. A., Yamaguchi A., Johnson D., Bevan A. W. R. and Burbine T. H. (2015) Geochemistry and oxygen isotope composition of maingroup pallasites and olivine-rich clasts in mesosiderites: Implications for the "Great Dunite Shortage" and HED-mesosiderite connection. Geochim. Cosmochim. Acta. 169, 115-136.

Greenwood R. C., Franchi I. A., Jambon A., Barrat J. A. and Burbine T. H. (2006) Oxygen isotope variation in stony-iron meteorites. Science 313, 1763-1765.

Grimm R. E. and Mcsween H. Y. (1993) Heliocentric zoning of the asteroid belt by aluminum-26 heating. In Lunar and Planetary Science Conference XXIV pp. 577-578.

Gueguen B., Rouxel O., Ponzevera E., Bekker A. and Fouquet Y. (2013) Nickel isotope variations in terrestrial silicate rocks and geological reference materials measured by MC-ICP-MS. Geostand. Geoanalytical Res. 37, 297-317. 
Haack H., Bizzarro M., Backer J. A. and Rosing M. (2003) Early thermal evolution and sizes of the HED and mesosiderite parent bodies new constraints from Lu-Hf chronology. In Lunar Planet. Sci. XXXIV. League City, Texas. \#1317(abstr.).

Hassanzadeh J., Rubin A. E. and Wasson J. T. (1990) Compositions of large metal nodules in mesosiderites: Links to iron meteorite group IIIAB and the origin of mesosiderite subgroups. Geochim. Cosmochim. Acta 54, 3197-3208.

Van Heghe L., Engström E., Rodushkin I., Cloquet C. and Vanhaecke F. (2012) Isotopic analysis of the metabolically relevant transition metals $\mathrm{Cu}, \mathrm{Fe}$ and $\mathrm{Zn}$ in human blood from vegetarians and omnivores using multi-collector ICP-mass spectrometry. J. Anal. At. Spectrom. 27, 1327-1334.

Hezel D. C., Poole G. M., Hoyes J., Coles B. J., Unsworth C., Albrecht N., Smith C., Rehkämper M., Pack A., Genge M. and Russell S. S. (2015) Fe and O isotope composition of meteorite fusion crusts: possible natural analogues to chondrule formation? Meteorit. Planet. Sci. 50, 229-242.

Hoefs J. (2009) Stable Isotope Geochemistry. 6th ed. Springer Berlin Heidelberg.

Jaoul O., Bertran-Alvarez Y., Liebermann R. C. and Price G. D. (1995) Fe-Mg interdiffusion in olivine up to 9 $\mathrm{GPa}$ at $\mathrm{T}=600-900^{\circ} \mathrm{C}$; experimental data and comparison with defect calculations. Phys. Earth Planet. Inter. 89, 199-218.

Lazar C., Young E. D. and Manning C. E. (2012) Experimental determination of equilibrium nickel isotope fractionation between metal and silicate from $500^{\circ} \mathrm{C}$ to $950^{\circ} \mathrm{C}$. Geochim. Cosmochim. Acta 86, 276-295.

Lodders K. (2003) Solar System Abundances and Condensation Temperatures of the Elements. Astrophys. J. 591, 1220-1247.

Luais B. (2007) Isotopic fractionation of germanium in iron meteorites: Significance for nebular condensation, core formation and impact processes. Earth Planet. Sci. Lett. 262, 21-36.

Maréchal C. N., Télouk P. and Albarède F. (1999) Precise analysis of copper and zinc isotopic compositions by plasma-source mass spectrometry. Chem. Geol. 156, 251-273.

Markowski A., Quitté G., Halliday A. and Kleine T. (2006) Tungsten isotopic compositions of iron meteorites: Chronological constraints vs. cosmogenic effects. Earth Planet. Sci. Lett. 242, 1-15.

Mason B. (1967) The Woodbine meteorite, with notes on silicates in iron meteorites. Mineral. Mag. 36, 120126.

McKibbin S. J., Ireland T. R., Holden P., O’Neill H. S. C. and Mallmann G. (2016) Rapid cooling of planetesimal core-mantle reaction zones from $\mathrm{Mn}-\mathrm{Cr}$ isotopes in pallasites. Geochemical Perspect. 2, 6877.

McKibbin S. J., O’Neill H. S. C., Mallmann G. and Halfpenny A. (2013) LA-ICP-MS mapping of olivine from the Brahin and Brenham meteorites: Complex elemental distributions in the pallasite olivine precursor. Geochim. Cosmochim. Acta 119, 1-17.

McSween H. Y., Mittlefehldt D. W., Beck A. W., Mayne R. G. and McCoy T. J. (2011) HED Meteorites and Their Relationship to the Geology of Vesta and the Dawn Mission. Space Sci. Rev. 163, 141-174. 
Miyamoto M. (1997) Chemical zoning of olivine in several pallasites. J. Geophys. Res. 102, 21,613-21,618.

Moynier F., Blichert-Toft J., Telouk P., Luck J.-M. and Albarède F. (2007) Comparative stable isotope geochemistry of Ni, $\mathrm{Cu}, \mathrm{Zn}$, and $\mathrm{Fe}$ in chondrites and iron meteorites. Geochim. Cosmochim. Acta 71, 4365-4379.

Mueller T., Watson E. B., Trail D., Wiedenbeck M., Van Orman J. and Hauri E. H. (2014) Diffusive fractionation of carbon isotopes in $\gamma$-Fe: Experiment, models and implications for early solar system processes. Geochim. Cosmochim. Acta 127, 57-66.

Mueller T., Watson E. B., Harrison T. M. (2010) Applications of diffusion data to high-temperature earth systems. Reviews in Mineralogy \& Geochemistry 72, 997-1038.

Oeser M., Dohmen R., Horn I., Schuth S. and Weyer S. (2015) Processes and time scales of magmatic evolution as revealed by $\mathrm{Fe}-\mathrm{Mg}$ chemical and isotopic zoning in natural olivines. Geochim. Cosmochim. Acta 154, $130-150$.

O’Neil J. R. (1986) Theoretical and experimental aspects of isotopic fractionation. Rev. Mineral. 16, 1-40.

Pernicka E. and Wasson J. T. (1987) Ru, Re, Os, Pt and Au in iron meteorites. Geochim. Cosmochim. Acta 51, $1717-1726$.

Poitrasson F., Halliday A. N., Lee D.-C., Levasseur S. and Teutsch N. (2004) Iron isotope differences between Earth, Moon, Mars and Vesta as possible records of contrasted accretion mechanisms. Earth Planet. Sci. Lett. 223, 253-266.

Poitrasson F., Levasseur S. and Teutsch N. (2005) Significance of iron isotope mineral fractionation in pallasites and iron meteorites for the core-mantle differentiation of terrestrial planets. Earth Planet. Sci. Lett. 234, $151-164$

Polyakov V. B. (2009) Equilibrium Iron Isotope Fractionation at Core-Mantle Boundary Conditions. Science 323, $912-914$.

Polyakov V. B. and Mineev S. D. (2000) The use of Mössbauer spectroscopy in stable isotope geochemistry. Geochim. Cosmochim. Acta 64, 849-865.

Porter S. J., Selby D. and Cameron V. (2014) Characterising the nickel isotopic composition of organic-rich marine sediments. Chem. Geol. 387, 12-21.

Powell B. N. (1969) Petrology and chemistry of mesosiderites-I. Textures and composition of nickel-iron. Geochim. Cosmochim. Acta 33, 789-810.

Quitté G., Birck J.-L. and Allègre C. J. (2005) Stony-iron meteorites: History of the metal phase according to tungsten isotopes. Geochim. Cosmochim. Acta 69, 1321-1332.

Rasmussen K. L., Malvin D. J., Buchwald V. F. and Wasson J. T. (1984) Compositional trends and cooling rates of group IVB iron meteorites. Geochim. Cosmochim. Acta 48, 805-813.

Rasmussen K. L., Malvin D. J. and Wasson J. T. (1988) Trace Element Partitioning between Taenite and Kamacite; Relationship to the Cooling Rates of Iron Meteorites. Meteoritics 23, 107-112. 
Ratié G., Jouvin D., Garnier J., Rouxel O., Miska S., Guimarães E., Cruz Vieira L., Sivry Y., Zelano I., Montarges-Pelletier E., Thil F. and Quantin C. (2015) Nickel isotope fractionation during tropical weathering of ultramafic rocks. Chem. Geol. 402, 68-76.

Regelous M., Elliott T. and Coath, C. D. (2008) Nickel isotope heterogeneity in the early Solar System. Earth Planet. Sci. Lett. 272, 330-338.

Richter F., Dauphas N. and Teng F. (2009) Non-traditional fractionation of non-traditional isotopes: Evaporation, chemical diffusion and Soret diffusion. Chem. Geol. 258, 92-103.

Richter F. M., Huss, G. R. and Mendybaev R. A. (2014) Iron and nickel isotopic fractionation across metal grains from three $\mathrm{CBb}$ meteorites. 45th Lunar and Planetary Science Conference, Texas. \#1346 (abstr.)

Richter F. M., Watson E. B., Mendybaev R., Dauphas N., Georg B., Watkins J. and Valley J. (2009) Isotopic fractionation of the major elements of molten basalt by chemical and thermal diffusion. Geochim. Cosmochim. Acta 73, 4250-4263.

Ruzicka A. (2014) Silicate-bearing iron meteorites and their implications for the evolution of asteroidal parent bodies. Chemie der Erde - Geochemistry 74, 3-48.

Ruzicka A., Boynton W. V. and Ganguly J. (1994) Olivine coronas, metamorphism, and the thermal history of the Morristown and Emery mesosiderites. Geochim. Cosmochim. Acta 58, 2725-2741.

Ruzicka A. and Hutson M. (2010) Comparative petrology of silicates in the Udei Station (IAB) and Miles (IIE) iron meteorites: Implications for the origin of silicate-bearing irons. Geochim. Cosmochim. Acta 74, 394433.

Schoenberg R. and Blanckenburg F. von (2006) Modes of planetary-scale Fe isotope fractionation. Earth Planet. Sci. Lett. 252, 342-359.

Schwandt C. S., Cygan R. T. and Westrich H. R. (1998) Magnesium self-diffusion in orthoenstatite. Contrib. to Mineral. Petrol. 130, 390-396.

Scott E. R. D. (1972) Chemical fractionation in iron meteorites and its interpretation. Geochim. Cosmochim. Acta 36, 1205-1236.

Scott E. R. D. (1977a) Composition, mineralogy and origin of group IC iron meteorites. Earth Planet. Sci. Lett. 37, 273-284.

Scott E. R. D. (1977b) Geochemical relationships between some pallasites and iron meteorites. Mineral. Mag. 41, 265-272.

Scott E. R. D. (1977c) Pallasites - metal composition, classification and relationships with iron meteorites. Geochim. Cosmochim. Acta 41, 349-360.

Scott E. R.D., Wasson J. T. and Buchwald V. F. (1973) The chemical classification of iron meteorites-VII. A reinvestigation of irons with Ge concentrations between 25 and 80 ppm. Geochim. Cosmochim. Acta 37, 1957-1983.Shahar A., Young E. D. and Manning C. E. (2008) Equilibrium high-temperature Fe isotope 
fractionation between fayalite and magnetite: An experimental calibration. Earth Planet. Sci. Lett. 268, $330-338$.

Sio C. K. I., Dauphas N., Teng F.-Z., Chaussidon M., Helz R. T. and Roskosz M. (2013) Discerning crystal growth from diffusion profiles in zoned olivine by in situ $\mathrm{Mg}-\mathrm{Fe}$ isotopic analyses. Geochim. Cosmochim. Acta 123, 302-321.

Steele R. C. J., Elliott T., Coath C. D. and Regelous M. (2011) Confirmation of mass-independent Ni isotopic variability in iron meteorites. Geochim. Cosmochim. Acta 75, 7906-7925.

Stewart B. W., Papanastassiou D. A. and Wasserburg G. J. (1994) Sm-Nd chronology and petrogenesis of mesosiderites. Geochim. Cosmochim. Acta 58, 3487-3509.

Tang H. and Dauphas N. (2012) Abundance, distribution, and origin of 60Fe in the solar protoplanetary disk. Earth Planet. Sci. Lett. 359-360, 248-263.

\section{Tang H. and Dauphas N. (2014) ${ }^{60} \mathrm{Fe}-{ }^{60} \mathrm{Ni}$ chronology of core formation in Mars. Earth Planet. Sci. Lett. 390 ,} 264-274.

Tanimizu M. and Hirata T. (2006) Determination of natural isotopic variation in nickel using inductively coupled plasma mass spectrometry. J. Anal. At. Spectrom. 21, 1423-1426.

Telus M., Dauphas N., Moynier F., Tissot F. L. H., Teng F.-Z., Nabelek P. I., Craddock P. R. and Groat L. A. (2012) Iron, zinc, magnesium and uranium isotopic fractionation during continental crust differentiation: The tale from migmatites, granitoids, and pegmatites. Geochim. Cosmochim. Acta 97, 247-265.

Telus M., Huss G. R., Ogliore R. C., Nagashima K., Howard D. L., Newville M. G. and Tomkins A. G. (2016) Mobility of Iron and Nickel at Low Temperatures: Implications for 60Fe-60Ni Systematics of Chondrules from Unequilibrated Ordinary Chondrites. Geochim. Cosmochim. Acta 178, 87-105.

Teng F.-Z., Dauphas N. and Helz R. T. (2008) Iron Isotope Fractionation During Magmatic Differentiation in Kilauea Iki Lava Lake. Science 320, 1620-1622.

Tomiyama T. and Huss G. R. (2006) Minor and Trace Element Zoning in Pallasite Olivine: Modeling Pallasite Thermal History. In Lunar Planet. Sci. XXXVII. League City, Texas. \#2132(abstr.).

Tomiyama T. and Huss G. R. (2005) Minor element behavior of pallasite olivine: understandidng pallasite thermal history and chronology. In Lunar Planet. Sci. XXXVI. League City, Texas. p. \#2071(abstr.).

Vanhaecke F. and Moens L. (2004) Overcoming spectral overlap in isotopic analysis via single- and multicollector ICP-mass spectrometry. Anal. Bioanal. Chem. 378, 232-240.

Wang K., Jacobsen S. B., Sedaghatpour F., Chen H. and Korotev R. L. (2015) The earliest Lunar Magma Ocean differentiation recorded in Fe isotopes. Earth Planet. Sci. Lett. 430, 202-208.

Wang K., Moynier F., Dauphas N., Barrat J.-A., Craddock P. and Sio C. K. (2012) Iron isotope fractionation in planetary crusts. Geochim. Cosmochim. Acta 89, 31-45.

Wasson J. T. (1970a) Ni, Ga, Ge and Ir in the metal of iron-meteorites-with-silicate-inclusions. Geochim. Cosmochim. Acta 34, 957-964. 
Wasson J. T. (1970b) The chemical classification of iron meteorites. Icarus 12, 407-423.

Wasson J. T. (1999) Trapped melt in IIIAB irons; solid/liquid elemental partitioning during the fractionation of the IIIAB magma. Geochim. Cosmochim. Acta 63, 2875-2889.

Wasson J. T. and Choi B.-G. (2003) Main-group pallasites: Chemical composition, relationship to IIIAB irons, and origin. Geochim. Cosmochim. Acta 67, 3079-3096.

Wasson J. T. and Kallemeyn G. (2002) the IAB iron-meteorite complex: A group, five subgroups, numerous grouplets, closely related, mainly formed by crystal segregation in rapidly cooling melts. Geochim. Cosmochim. Acta 66, 2445-2473.

Wasson J. T. and Rubin A. E. (1985) Formation of mesosiderites by low-velocity impacts as a natural consequence of planet formation. Nature 318, 168-170.

Wasson J. T., Schaudy R., Bild R. W. and Chou C.-L. (1974) Mesosiderites-I. Compositions of their metallic portions and possible relationship to other metal-rich meteorite groups. Geochim. Cosmochim. Acta 38, 135-149.

Watson E. B. and Müller T. (2009) Non-equilibrium isotopic and elemental fractionation during diffusioncontrolled crystal growth under static and dynamic conditions. Chem. Geol. 267, 111-124.

Wasylenki L. E., Howe H. D., Spivak-Birndorf L. J. and Bish D. L. (2015) Ni isotope fractionation during sorption to ferrihydrite: Implications for $\mathrm{Ni}$ in banded iron formations. Chem. Geol. 400, 56-64.

Weyer S. (2008) Geochemistry: What Drives Iron Isotope Fractionation in Magma? Science 320, 1600-1601.

Weyer S., Anbar A. D., Brey G. P., Munker C., Mezger K., Woodland A. B., Münker C., Mezger K. and Woodland A. B. (2005) Iron isotope fractionation during planetary differentiation. Earth Planet. Sci. Lett. 240, 251-264.

Weyrauch M., Zipfel J. and Weyer S. (2015) Ni isotope composition of zoned metal grains in CBb chondrite Hammadah al Hamra 237. Paneth Kolloquium, Nördlingen. \#0051 (abstr.)

Williams H. M., Markowski A., Quitté G., Halliday A. N., Teutsch N. and Levasseur S. (2006) Fe isotope fractionation in iron meteorites: New insights into metal-sulphide segregation and planetary accretion. Earth Planet. Sci. Lett. 250, 486-500.

Wombacher F. and Rehkamper M. (2003) Investigation of the mass discrimination of multiple collector ICP-MS using neodymium isotopes and the generalised power law. J. Anal. At. Spectrom. 18, 1371-1375.

Yang C.-W., Williams D. B. and Goldstein J. I. (1996) A revision of the Fe-Ni phase diagram at low temperatures $\left(<400{ }^{\circ} \mathrm{C}\right)$. Journal of Phase Equilibria 17, 522-531.

Yang J., Goldstein J. I. and Scott E. R. D. (2010) Main-group pallasites: Thermal history, relationship to IIIAB irons, and origin. Geochim. Cosmochim. Acta 74, 4471-4492. 
Young E. D., Galy A. and Nagahara H. (2002) Kinetic and equilibrium mass-dependent isotope fractionation laws in nature and their geochemical and cosmochemical significance. Geochim. Cosmochim. Acta 66, 1095-1104.

Young E. D., Manning C. E., Schauble E. A., Shahar A., Macris C. A., Lazar C. and Jordan M. (2015) Hightemperature equilibrium isotope fractionation of non-traditional stable isotopes: Experiments, theory, and applications. Chem. Geol. 395, 176-195.

Zhu X. K., Guo Y., O’Nions R. K., Young E. D. and Ash R. D. (2001) Isotopic homogeneity of iron in the early solar nebula. Nature 412, 311-313.

Zhu X. K., Guo Y., Williams R. J. P., O’Nions R. K., Matthews A., Belshaw N. S., Canters G. W., de Waal E. C., Weser U., Burgess B. K. and Salvato B. (2002) Mass fractionation processes of transition metal isotopes. Earth Planet. Sci. Lett. 200, 47-62. 


\section{Figure captions}

Figure 1. 2D Ni Ka intensity distribution maps of 3 thick sections of iron meteorites as obtained using $\mu \mathrm{XRF}$, black round spots represent micro-drilling sampling positions (see Table 1). The color code is given in relative intensities. Gibeon IVA (A) and Henbury IIIAB (B) iron meteorites demonstrate clear Widmanstätten patterns, while Canyon Diablo does not (C). Typical Ni concentration profiles for 3 iron meteorites are shown (marked by black bar in Fig. 1A and C). Higher Ni concentrations are predicted in taenite in contact with kamacite according to the phase diagram, so the concentrations shown here result from insufficient spatial resolution (Yang et al. 1996).

Figure 2. Isotope plots for $\delta^{56 / 54} \mathrm{Fe}$ and $\delta^{60 / 58} \mathrm{Ni}$. Open diamonds represent fusion crusts of the corresponding ordinary chondrites. The values for GRMs BHVO-1, OKUM and PCC-1 are taken from literature (Dauphas et al., 2009; Chernonozhkin et al., 2015). The data of $\delta^{56 / 54} \mathrm{Fe}$ in OKUM are from this work.

Figure 3. $\delta^{57 / 54} \mathrm{Fe}_{\text {metal }}$ vs $\delta^{57 / 54} \mathrm{Fe}_{\text {olivine }}$ plot for pallasites. Geothermometric isotherms for metal-olivine equilibration at different temperatures are calculated A) using parameters of Mössbauer spectroscopy for olivine and native iron (Polyakov and Mineev, 2000) and B) using parameters of NRIXS for olivine and $\gamma$-Fe (fcc) (Dauphas et al. 2012, Dauphas et al. 2014). The reference values for pallasites are taken from (Zhu et al., 2002; Poitrasson et al., 2005).

Figure 4. A. $\delta^{60 / 58} \mathrm{Ni}$ and $\delta^{57 / 54} \mathrm{Fe} v s$ Ir concentration in metal fractions of pallasites and IIIAB irons, suggested they share a genetic link. The detailed explanation of the trend line shown is provided in the ESI. B. The fractional crystallization trend for $\mathrm{Ni}$ isotopes is elusive, demonstrating the low impact of fractional crystallization on $\mathrm{Ni}$ isotopes. Additional literature data for $\mathrm{Fe}$ and $\mathrm{Ni}$ isotopic composition of meteorites are from (Zhu et al., 2001; Poitrasson et al., 2005; Weyer et al., 2005; Williams et al., 2006; Schoenberg and Blanckenburg, 2006; Cook et al., 2007). The data for bulk Ir content in meteorites are from (Scott et al., 1973; Wasson, 1999; Wasson and Choi, 2003; Danielson et al., 2009).

Figure 5. 2D element distribution map of Ni in olivine of Seymchan PMG. The map was produced in the direction from the metal-olivine margin (left side) to the core of the olivine (right side). The image dimension is 1460x600 $\mu \mathrm{m}$, and each pixel represents 20x20 $\mu \mathrm{m}$. The color bar shows the Ni concentration in $\mu \mathrm{g} \mathrm{g}^{-1}$.

Figure 6. Three-isotope plots based on data from the laterally resolved isotopic analyses of Fe and Ni in magmatic and silicate-bearing iron meteorites (microdrilled sub-samples). The experimental fractionation exponent $\beta_{\text {exp }}$ was extracted from the laterally resolved data of all single microdrilled iron meteorites using the approach described by Young et al. (Young et al., 2002). The slope of the regression line in $\delta^{\prime}-\delta^{\prime}$ three-isotope plot is equal to the fractionation exponent $\beta$ between the corresponding isotope ratios. By definition $\delta^{\prime x / 58} \mathrm{Ni}=$ $\ln \left[\left({ }^{\mathrm{x}} \mathrm{Ni} /{ }^{58} \mathrm{Ni}\right)_{\mathrm{smp}} /\left({ }^{\mathrm{x}} \mathrm{Ni} /{ }^{58} \mathrm{Ni}\right)_{\mathrm{std}}\right] \cdot 1000$.

Figure B1. Three-isotope plots for $\mathrm{Fe}$ and $\mathrm{Ni}$ isotope fractionation between metal and silicate in pallasites, mesosiderites and Udei Station iron meteorite with silicate inclusions.

Figure B2. A - frequency distribution of Ir in 150 IIIAB iron meteorites and 48 main group pallasites. Data from (Scott et al., 1973; Scott, 1977; Wasson, 1999; Wasson and Choi, 2003; Lauretta et al., 2006; van Niekerk et al., 2007; Danielson et al., 2009). B - frequency distribution of $\delta^{57 / 54} \mathrm{Fe}$, calculated using the Ir concentrations and the empirically fitted lines from figure B1. 
Figure B3. $\delta^{57 / 54} \mathrm{Fe}$ vs Ir concentration in metal fractions of pallasites and IIAB irons. Literature data for the Fe isotopic compositions of these meteorites are from (Zhu et al. 2001, Williams et al. 2006).

Figure C1. Three-isotope plots for $\mathrm{Fe}$ and $\mathrm{Ni}$ isotope fractionation between metal and silicate in pallasites, mesosiderites and Udei Station iron meteorite with silicate inclusions.

Figure D1. Fe and Ni isotopic composition of iron meteorites and selected mafic terrestrial igneous and sedimentary rocks. The Ni isotope data has previously been reported (Chernonozhkin et al., 2015) and the Fe isotopic composition corresponds to bulk digestions of the iron meteorites analyzed. The Fe isotopic compositions of GRMs were taken from literature (Dauphas et al., 2009, 2007; Fehr et al., 2008; Poitrasson et al., 2004; Telus et al., 2012; Wang et al., 2012). 
Tables and captions

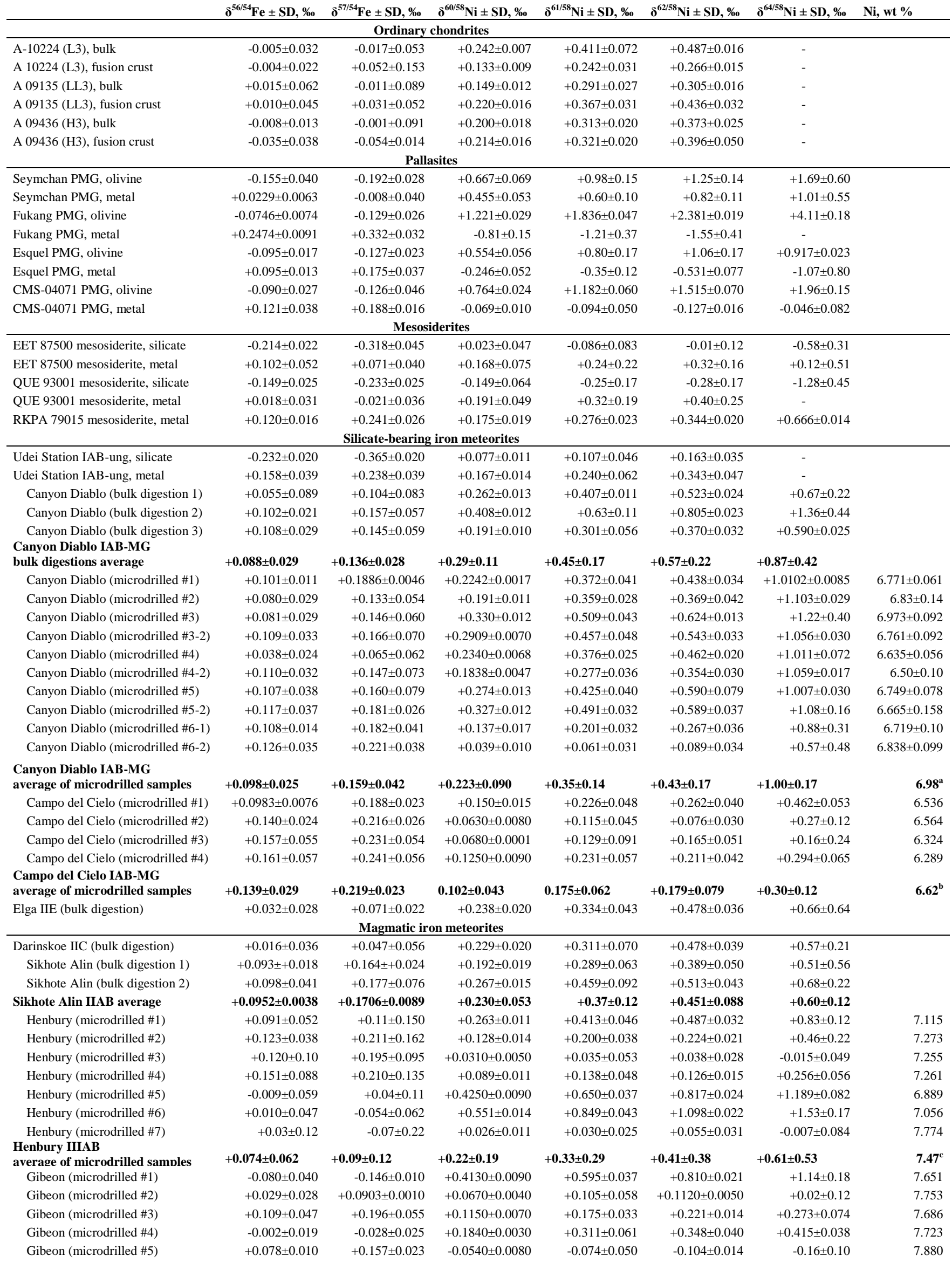




\begin{tabular}{|c|c|c|c|c|c|c|c|}
\hline Gibeon (microdrilled \#6) & $-0.04 \pm 0.11$ & $-0.064 \pm 0.043$ & $+0.2260 \pm 0.0030$ & $+0.350 \pm 0.033$ & $+0.485 \pm 0.028$ & $+0.61 \pm 0.14$ & 7.793 \\
\hline Gibeon (microdrilled \#7) & $+0.02 \pm 0.11$ & $-0.00 \pm 0.17$ & $-0.0005 \pm 0.0080$ & $+0.0390 \pm 0.0060$ & $+0.012 \pm 0.030$ & $-0.049 \pm 0.045$ & 7.809 \\
\hline \multicolumn{8}{|l|}{ Gibeon IV } \\
\hline Chinga (bulk digestion) & $+0.031 \pm 0.063$ & $+0.045 \pm 0.041$ & $+0.222 \pm 0.015$ & $+0.329 \pm 0.064$ & $+0.476 \pm 0.042$ & $+0.77 \pm 0.38$ & \\
\hline Chinga (microdrilled \#1) & $+0.025 \pm 0.014$ & $+0.061 \pm 0.044$ & $+0.248 \pm 0.015$ & $+0.385 \pm 0.028$ & $+0.513 \pm 0.037$ & $+1.04 \pm 0.13$ & $16.48 \pm 0.22$ \\
\hline Chinga (microdrilled \#2) & $+0.038 \pm 0.028$ & $+0.080 \pm 0.017$ & $+0.239 \pm 0.013$ & $+0.393 \pm 0.010$ & $+0.527 \pm 0.025$ & $+0.950 \pm 0.042$ & $16.52 \pm 0.10$ \\
\hline \multirow[t]{2}{*}{ Chinga IVB-an average } & $+0.0314 \pm 0.0065$ & $+0.062 \pm 0.018$ & $+0.236 \pm 0.013$ & $+0.369 \pm 0.035$ & $+0.505 \pm 0.026$ & $+0.92 \pm 0.14$ & $16.5^{\mathrm{e}}$ \\
\hline & & & Other materials & & & & \\
\hline OKUM komatiite CRM & $-0.003 \pm 0.029$ & $+0.040 \pm 0.054$ & $+0.580 \pm 0.070$ & $+0.680 \pm 0.070$ & $+1.13 \pm 0.13$ & - & \\
\hline BHVO-1 basalt CRM & $+0.138 \pm 0.013$ & $+0.207 \pm 0.041$ & $+0.081 \pm 0.034$ & $+0.19 \pm 0.20$ & $+0.130 \pm 0.093$ & $+0.14 \pm 0.10$ & \\
\hline PCC-1 peridotite CRM & $+0.048 \pm 0.060$ & $+0.111 \pm 0.096$ & $+0.166 \pm 0.048$ & $+0.25 \pm 0.14$ & $+0.342 \pm 0.065$ & $+0.62 \pm 0.39$ & \\
\hline
\end{tabular}

$\frac{\text { PCC-1 peridotite CRM }}{1}$ Table 1. Ni and Fe isotopic compositions of chondrites, stony-iron, magmatic iron, silicate-bearing iron

2 meteorites and reference materials. Every sample was measured 3 to 5 times during at least 2 different

3 measurement sessions. The results are presented in per mille deviations versus the corresponding reference

4 material (NIST SRM 986 for Ni and IRMM-014 for Fe). The Ni isotopic compositions in geological reference

5 materials and in bulk digests of iron meteorites have been reported previously (Chernonozhkin et al., 2015),

6 while the $\mathrm{Fe}$ isotopic composition was determined using the same solutions of these digested meteorites. The Ni

7 concentration data has been taken from the following references: a) (Scott, 1977a) b) (Wasson, 1970b) c)

8 (Pernicka and Wasson, 1987) d) (Rasmussen et al., 1988) and e) (Rasmussen et al., 1984) 


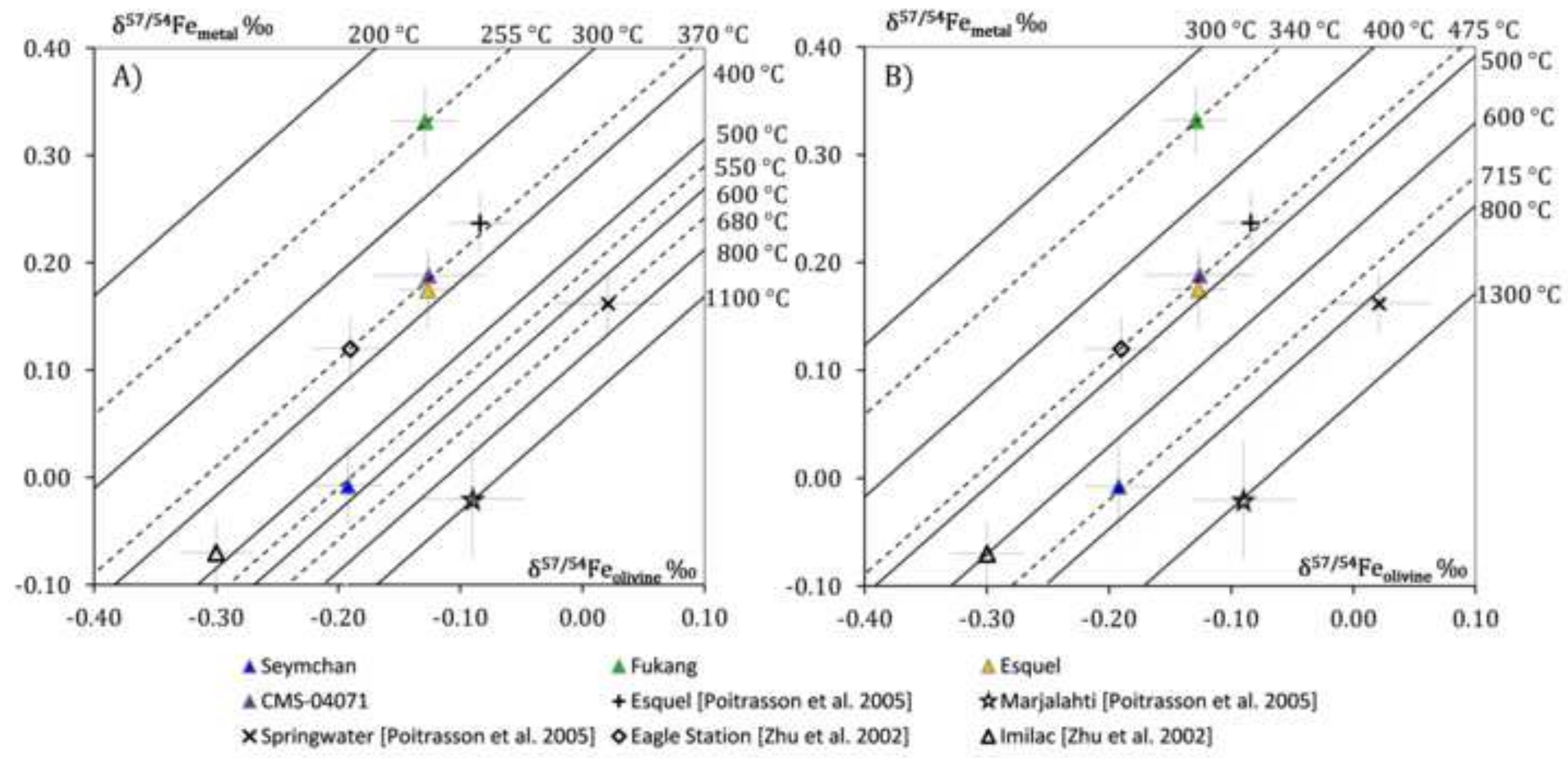


B

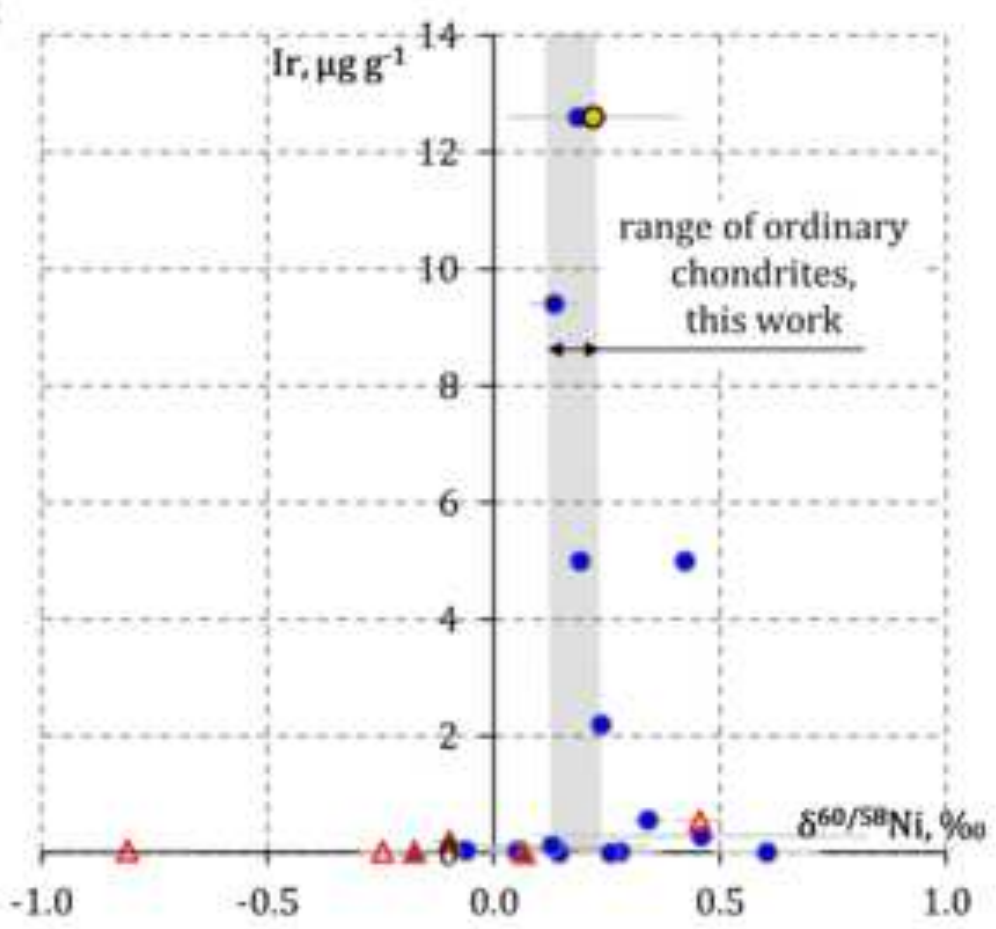

- IIIAB, ref.

A PMG, ref.

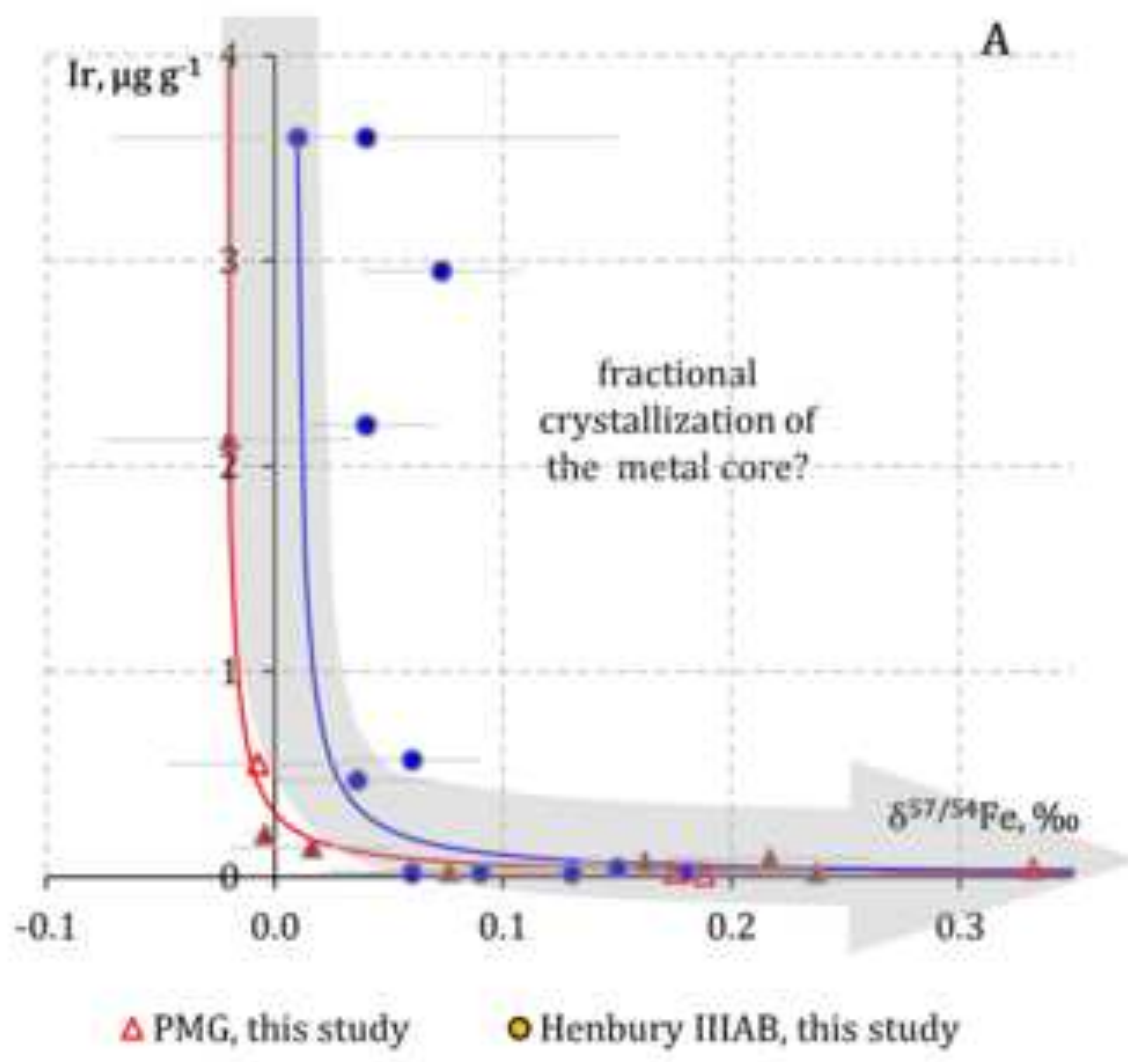


Equation 2
\[ \beta_{\text {kin }}=\frac{\ln \left(m_{58} / m_{62}\right)}{\ln \left(m_{58} / m_{60}\right)} \]

$$
\beta_{k i n}=\frac{\ln \left(m_{58} / m_{62}\right)}{\ln \left(m_{58} / m_{60}\right)}
$$

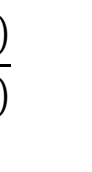

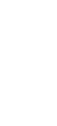

.

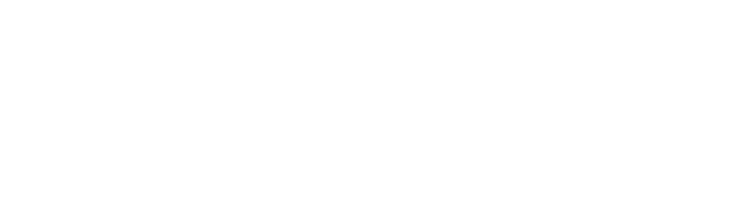

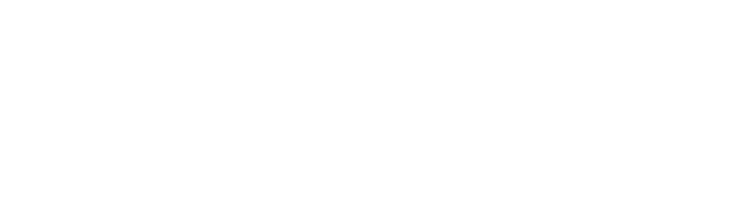




$$
\delta^{x / 58} \mathrm{Ni}=\left[\frac{\left({ }^{x} \mathrm{Ni} /{ }^{58} \mathrm{Ni}\right)_{\text {Cu corr }, \text { smp }}}{\left({ }^{x} \mathrm{Ni} /{ }^{58} \mathrm{Ni}\right)_{\text {Cu corr }, \text { NISTSRM } 986}}-1\right] \cdot 1,000
$$




$$
\delta^{x / 54} \mathrm{Fe}=\left[\frac{\left({ }^{x} \mathrm{Fe} /{ }^{54} \mathrm{Fe}\right)_{\mathrm{Ni} \text { corr }, \text { smp }}}{\left({ }^{x} \mathrm{Fe} /{ }^{54} \mathrm{Fe}\right)_{\mathrm{Ni} \text { corr }, \text { IRMM } 014}}-1\right] \cdot 1,000
$$

Equation 4 


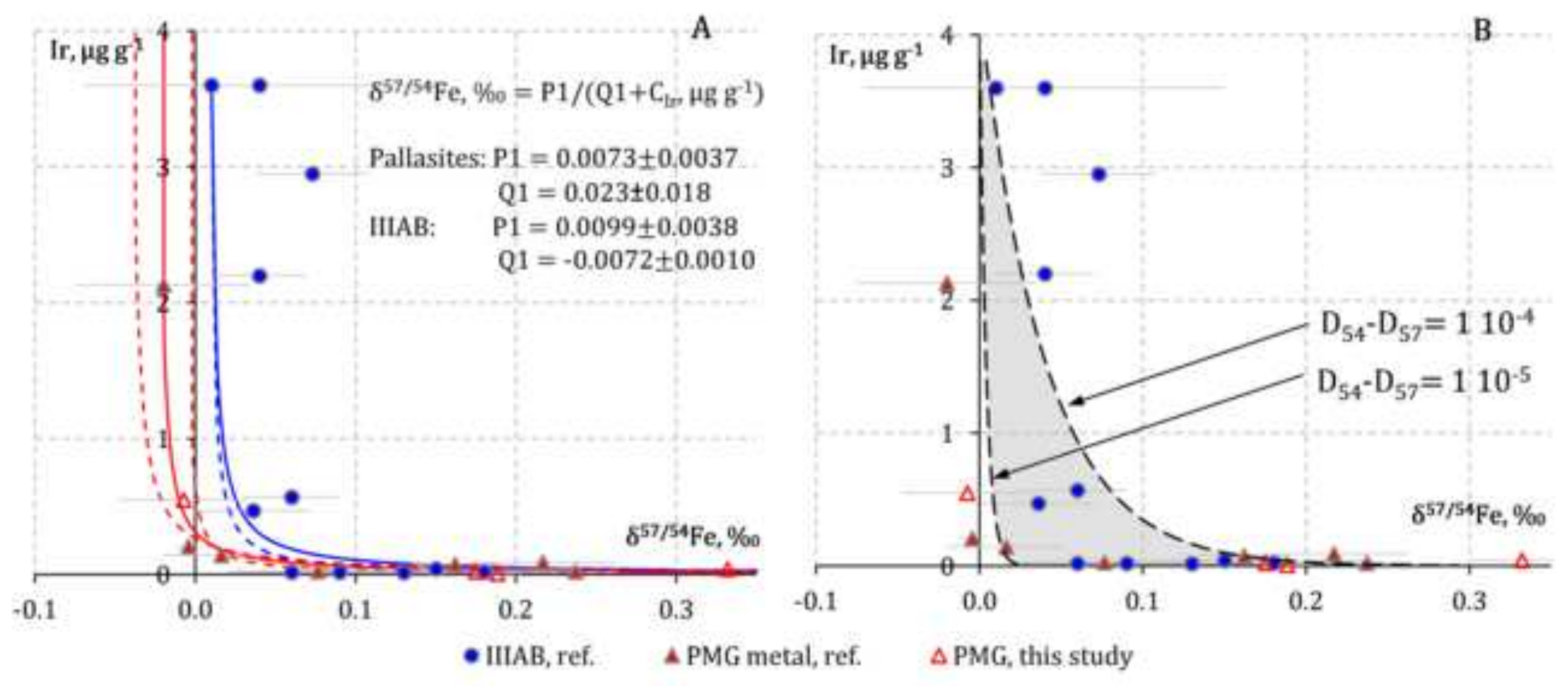



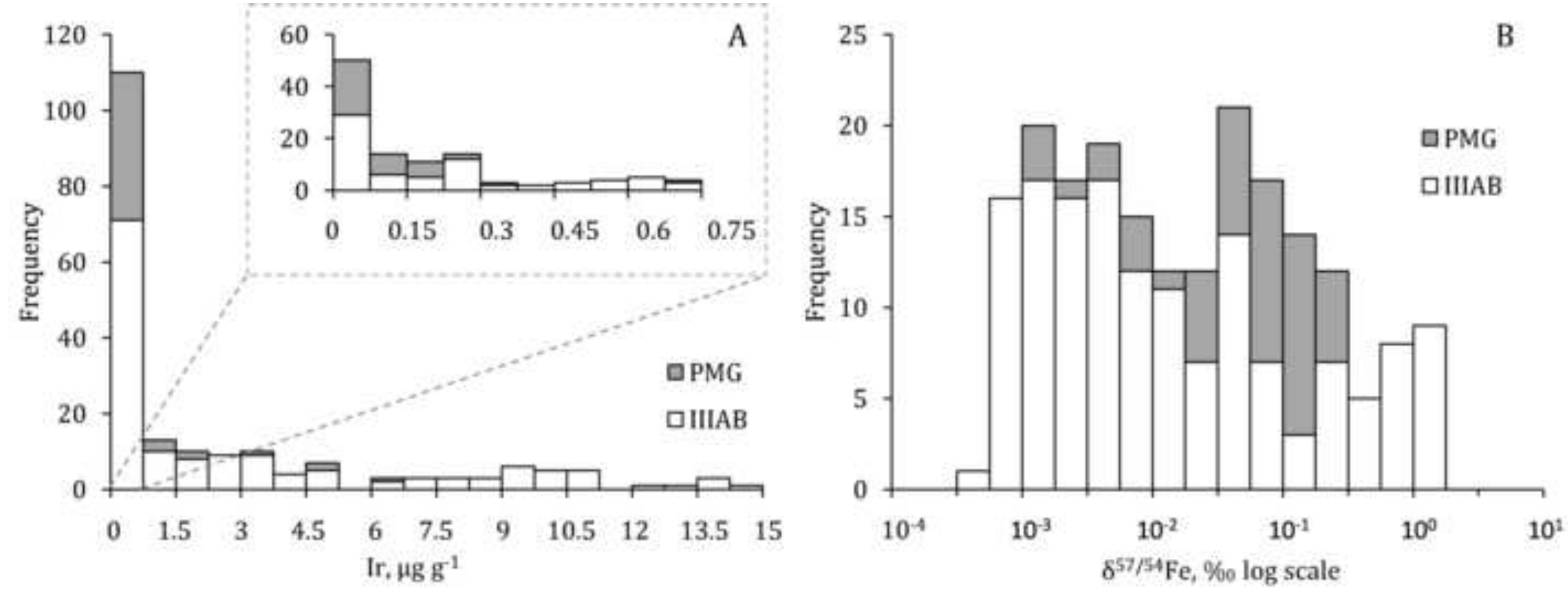


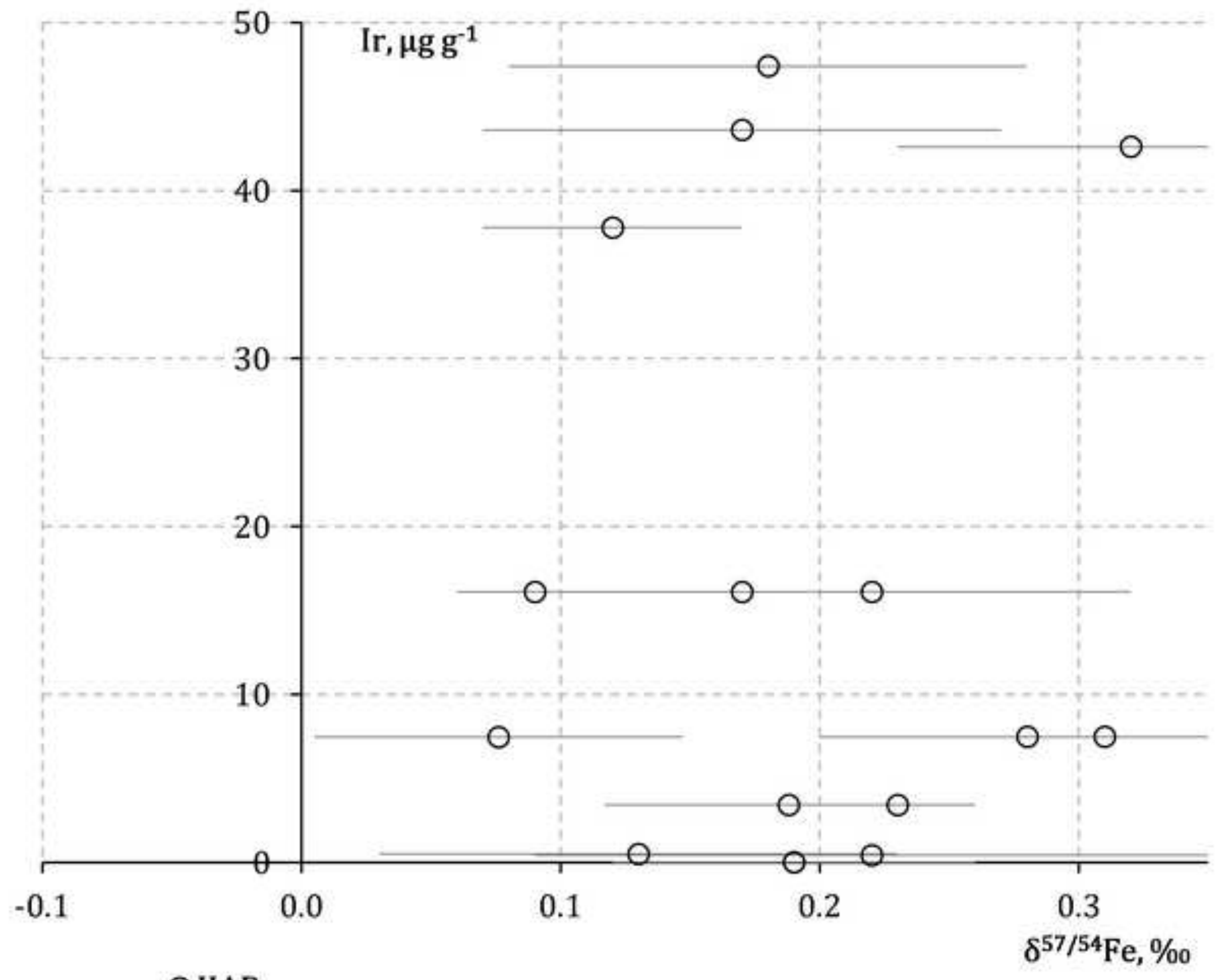

OIIAB 


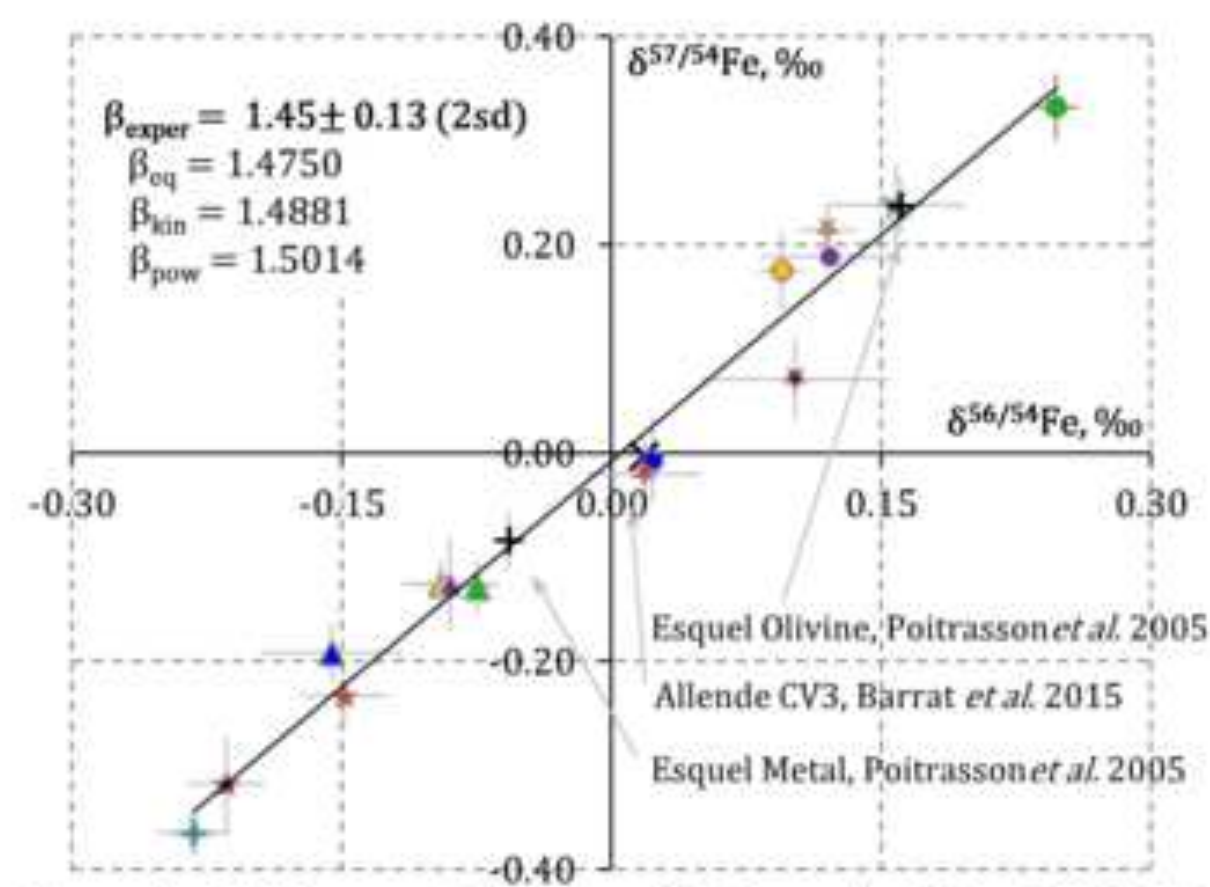

A Seymchan Olivine A Fukang Olivine

$\triangle$ Esquel Olivine

A. CMS-04071 Olivine

- EET-8750,51 Silicate

- QUE-93001,23 Silicate

+ Udei Station Silicate

* RKPA-7901 Metal

$\Delta_{\text {Mat. }} 56 / 4=0.178 \pm 0.092$ $\Delta_{\mathrm{mnc}} \mathrm{s}^{6 / 5 / 4}=0.322 \pm 0.033$ - Fukang Metal $\Delta_{\text {Met }} 56 / 4=0.190 \pm 0.060$

- Esquel Metal

$\Delta_{\text {Mers }}{ }^{56 / H}=0.21 \pm 0.13$

- CMS-04071 Metal

$\Delta_{\text {Mets }}=0.5 \% / 54=0.32 \pm 0.15$

- EET-8750,51 Metal

$\Delta_{M e t} 56 / 54=0.17 \pm 0.11$

- Udei Station Metal

$\Delta_{\text {Mat: }}$ s $^{56 / 54}=0.39 \pm 0.12$

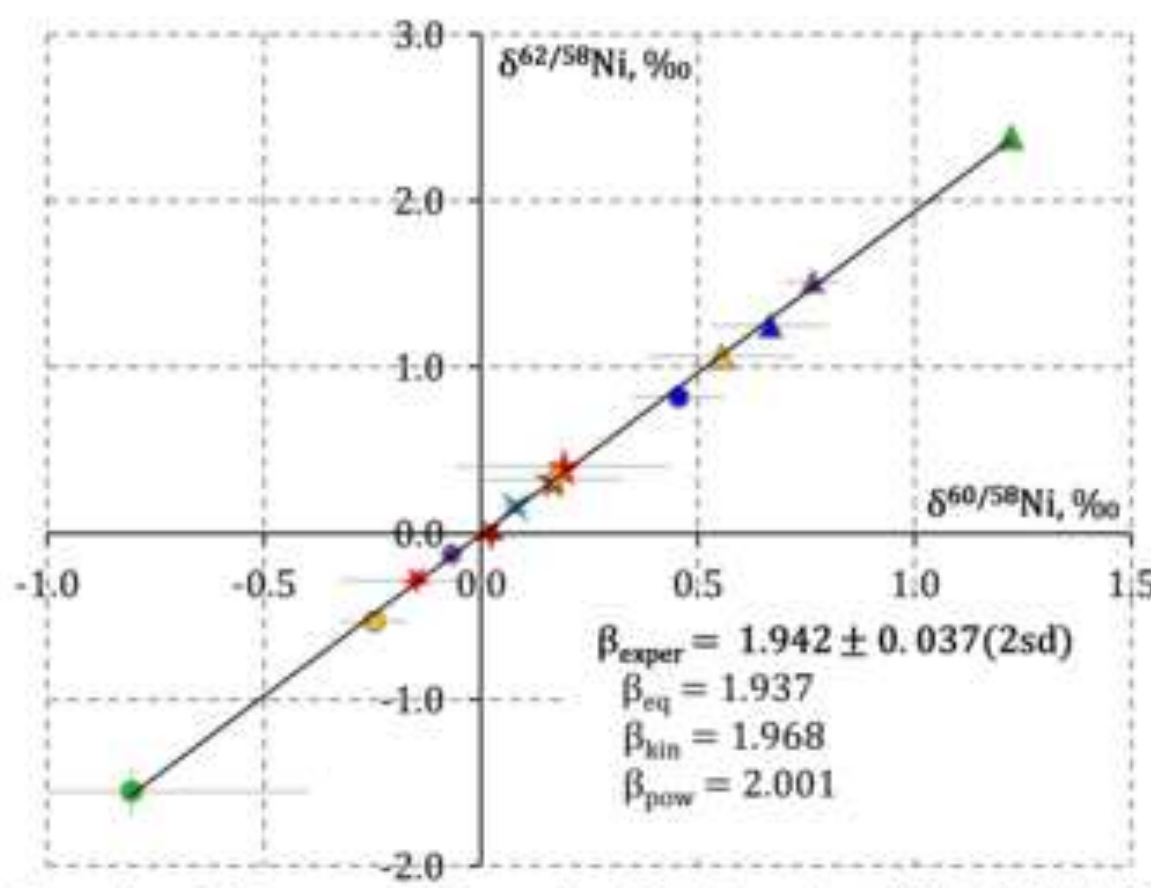

A Seymchan Olivine

A Fukang Olivine

$\triangle$ Esquel Olivine

A. CMS-04071, olivine

* QUE-93001,23 Silicate

+ Udei Station, metal

+ RKPA-79015, metal
- EET-8750,51 Silicate

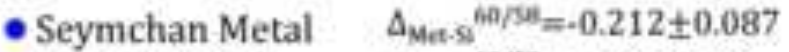
- Fukang Metal $\quad \Delta_{\text {Mets }}{ }^{60 / 58}=-2.03 \pm 0.16$

- Esquel Metal $\quad \Delta_{\text {Met. }}{ }^{60 / 58}=-0.800 \pm 0.077$

- CMS-04071, metal $\Delta_{\text {Mer.si }}{ }^{60 / 59}=-0833 \pm 0,026$

* EET-8750,51 Metal $\Delta_{\text {Met }}{ }^{60 / 58}=0.145 \pm 0.089$

* QUE-93001,23 Metal ${ }_{\Delta_{\text {Mes }}}{ }^{60 / 50}=0.340 \pm 0.080$

$\times$ Udei Station, Silicate $\Delta_{\text {Met: } 8}{ }^{60 / 58}=0.091 \pm 0.018$ 

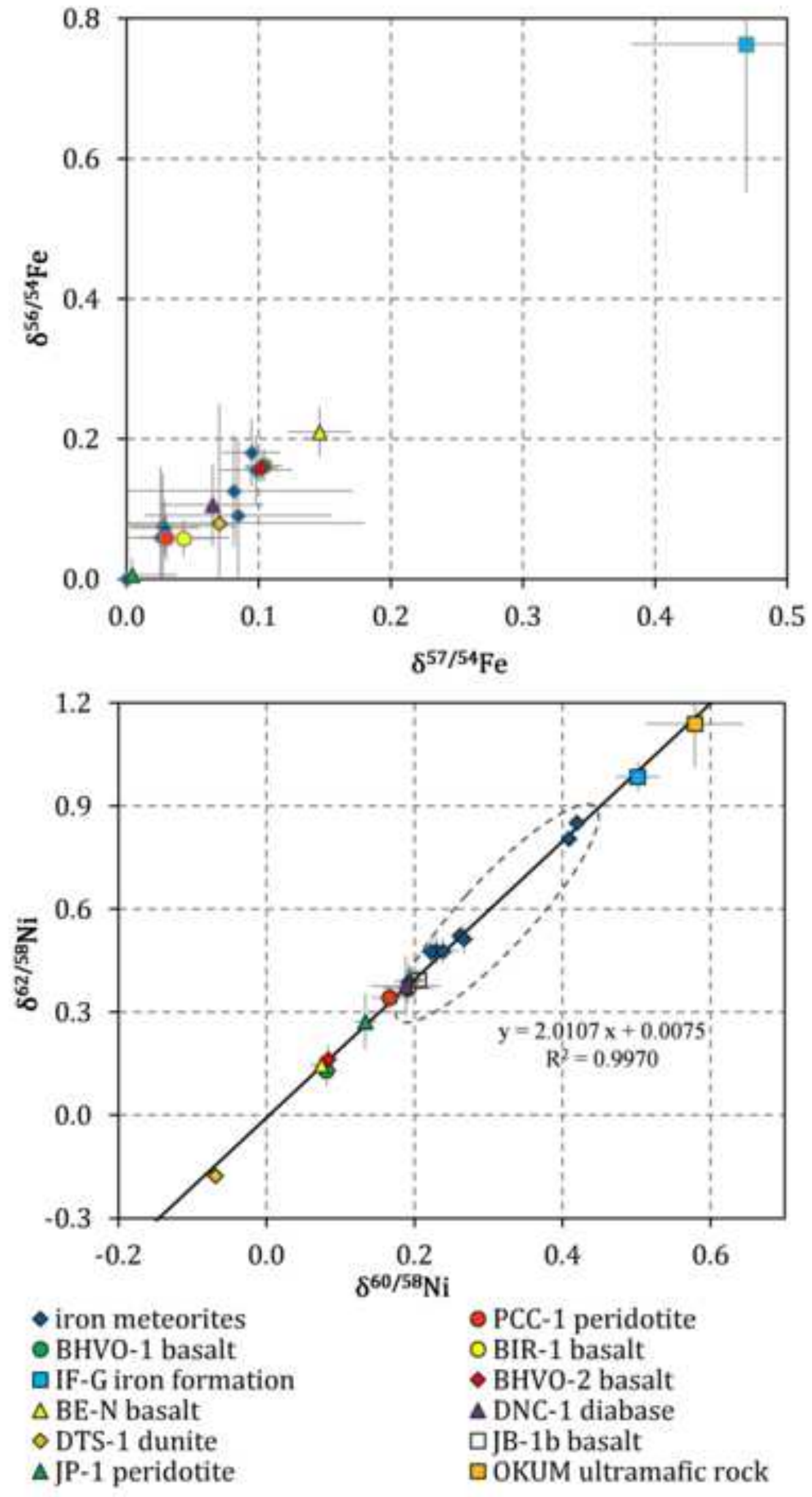


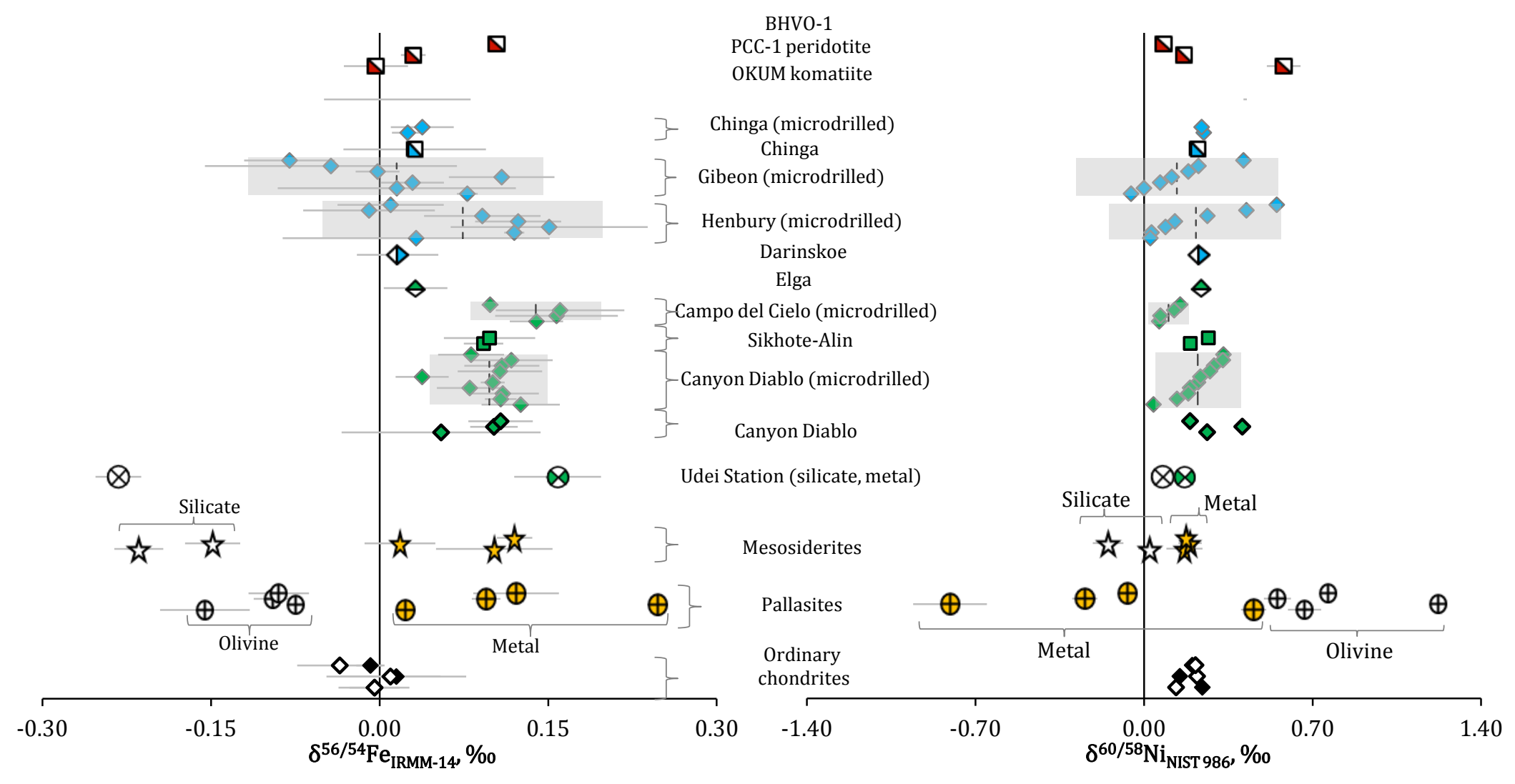




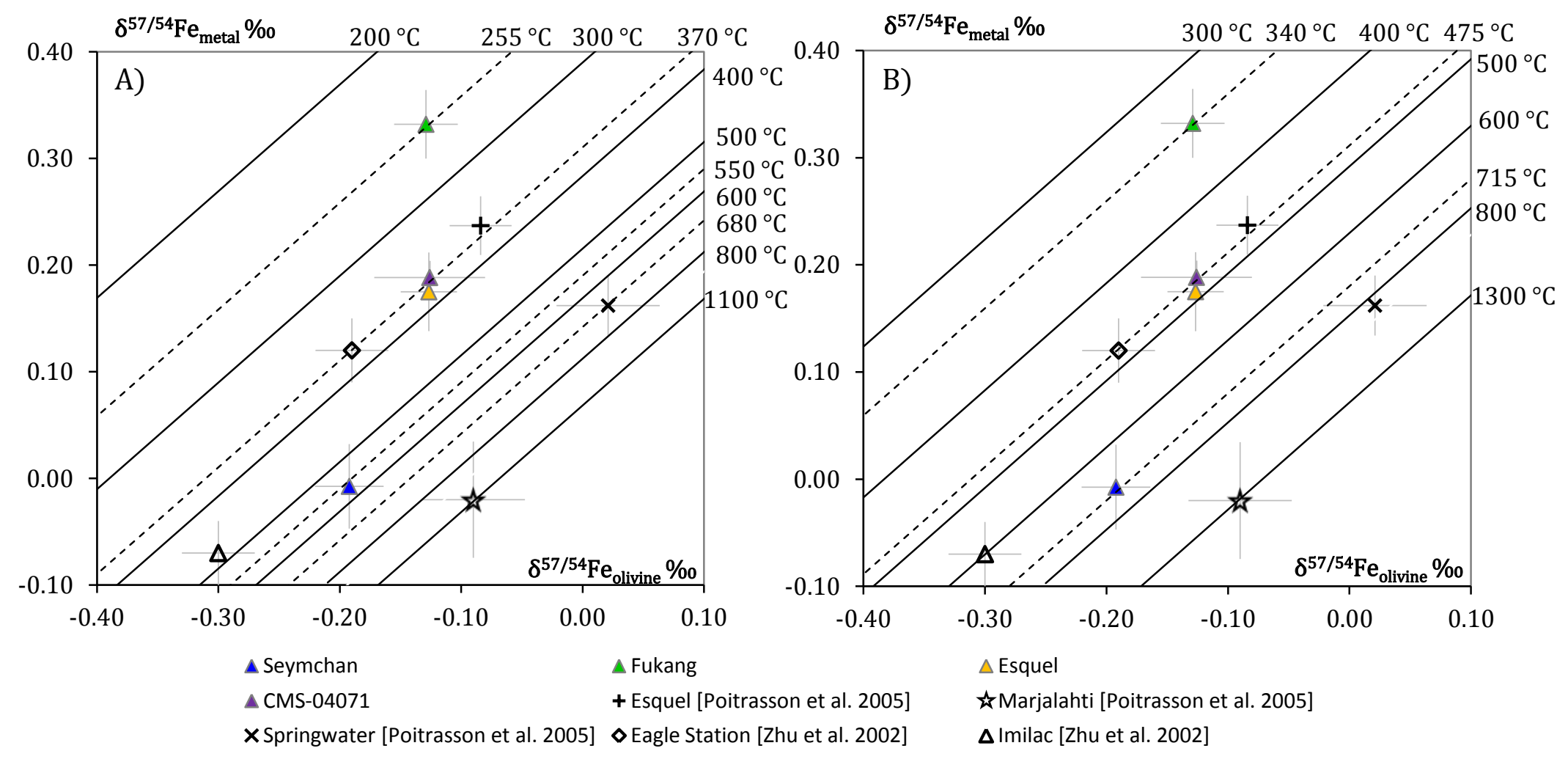



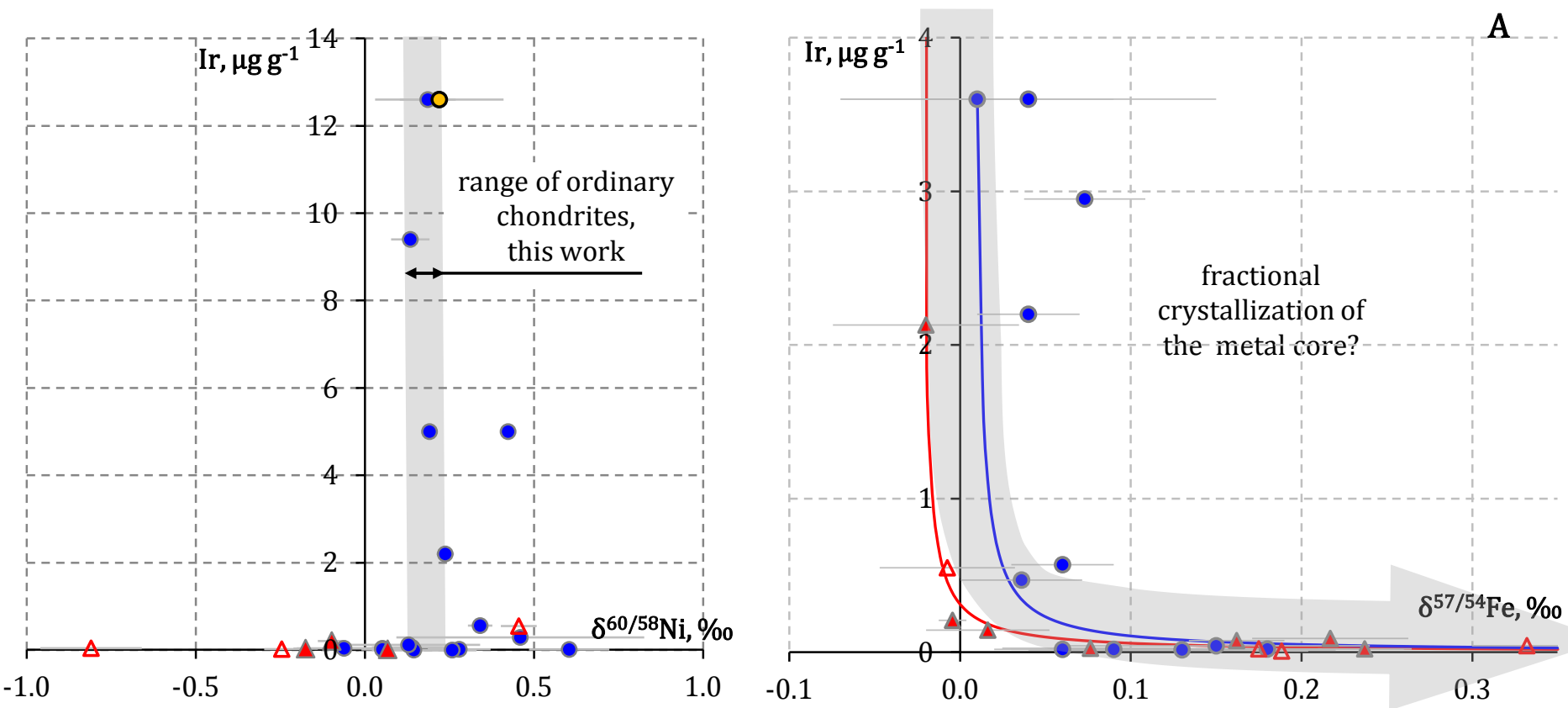

- IIIAB, ref.

$\triangle P M G$, ref.

$\triangle \mathrm{PMG}$, this study

o Henbury IIIAB, this study 


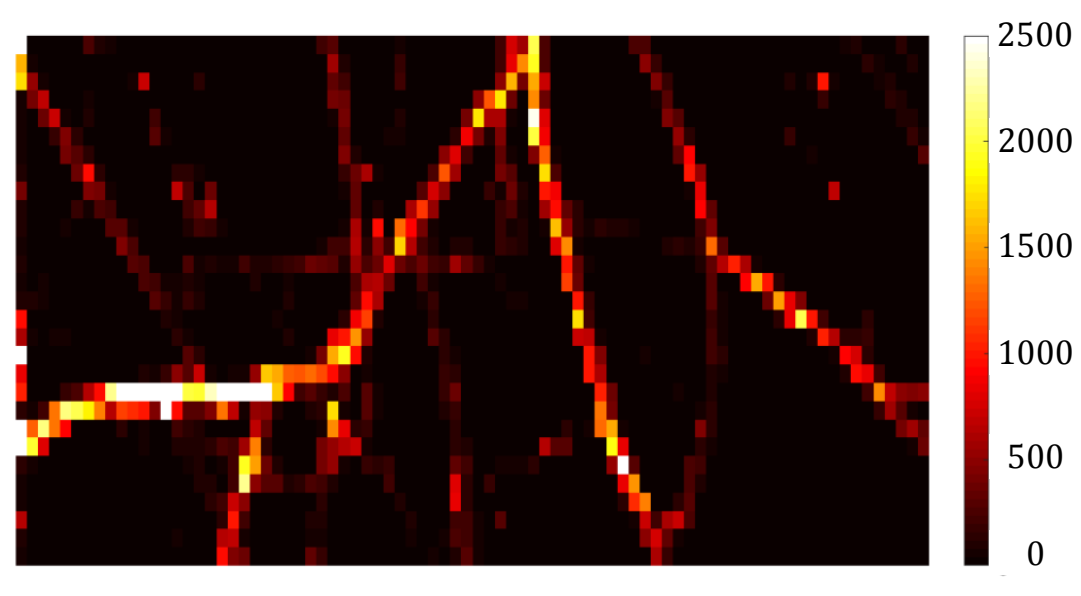



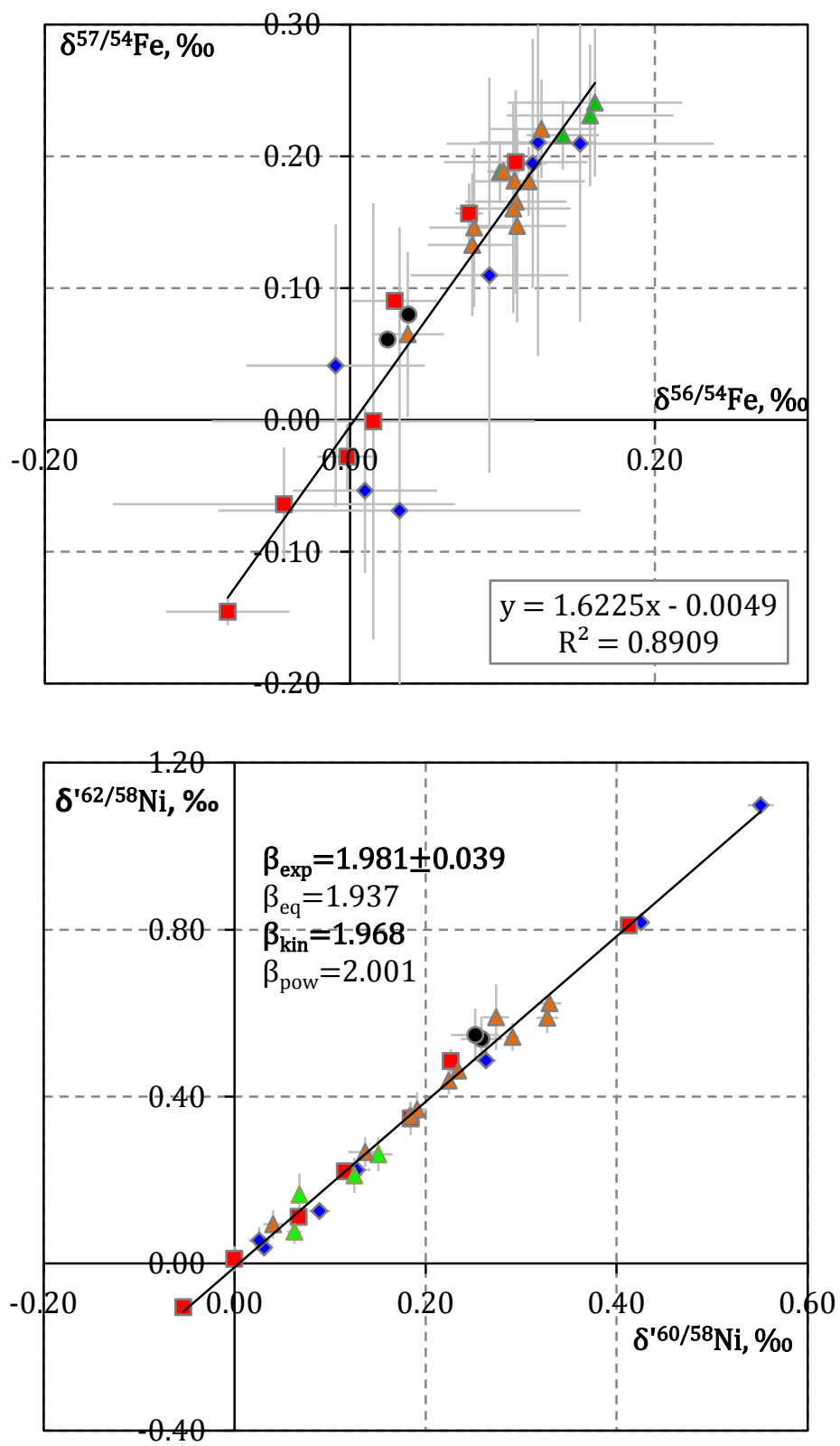

$\delta^{\prime 62 / 58} \mathrm{Ni}, \%=1.981( \pm 0.039,1 \mathrm{SD}) \delta^{\prime 60 / 58} \mathrm{Ni}, \% 0-0.086( \pm 0.089,1 \mathrm{SD})$ $\mathrm{R}^{2}=0.9895$

- Henbury IIIAB

Gibeon IVA

$\triangle$ Campo del Cielo IAB-MG

$\triangle$ Canyon Diablo IAB-MG

- Chinga ungrouped 

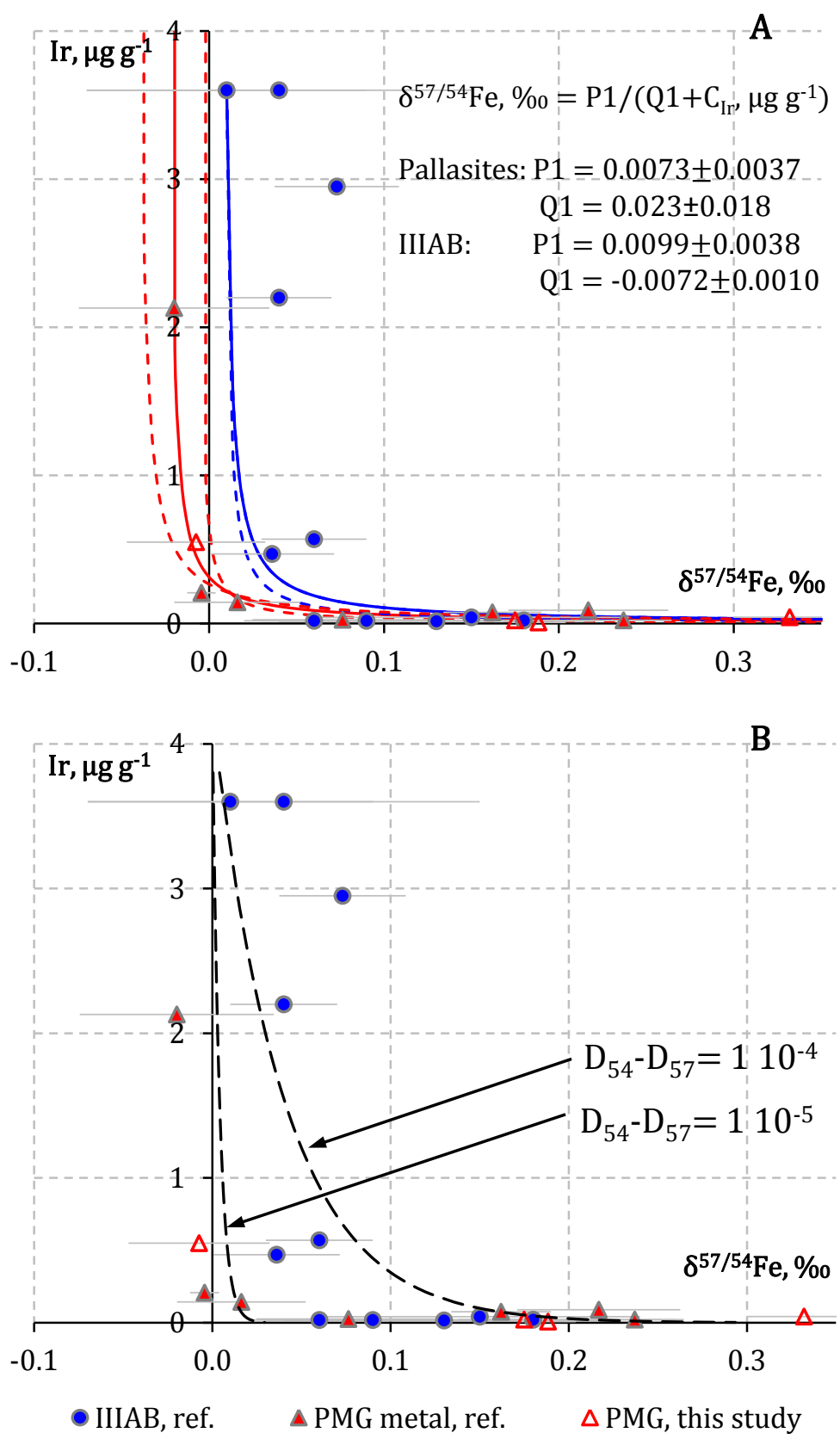

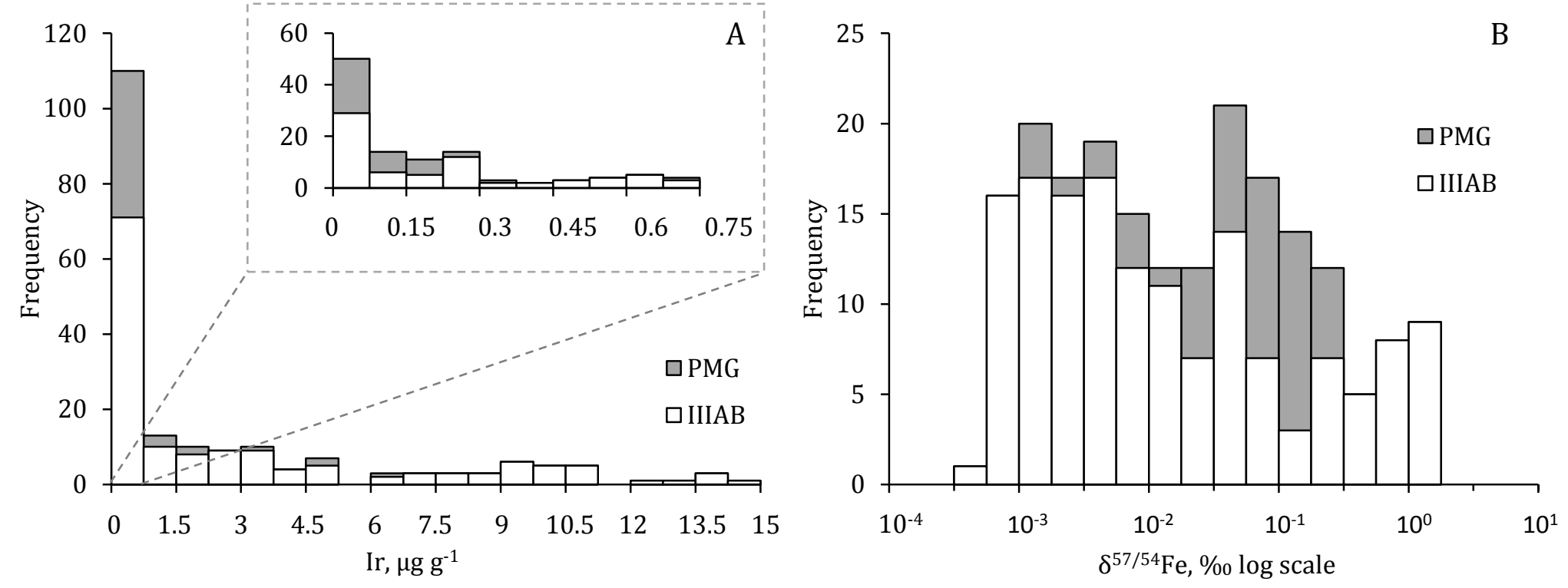


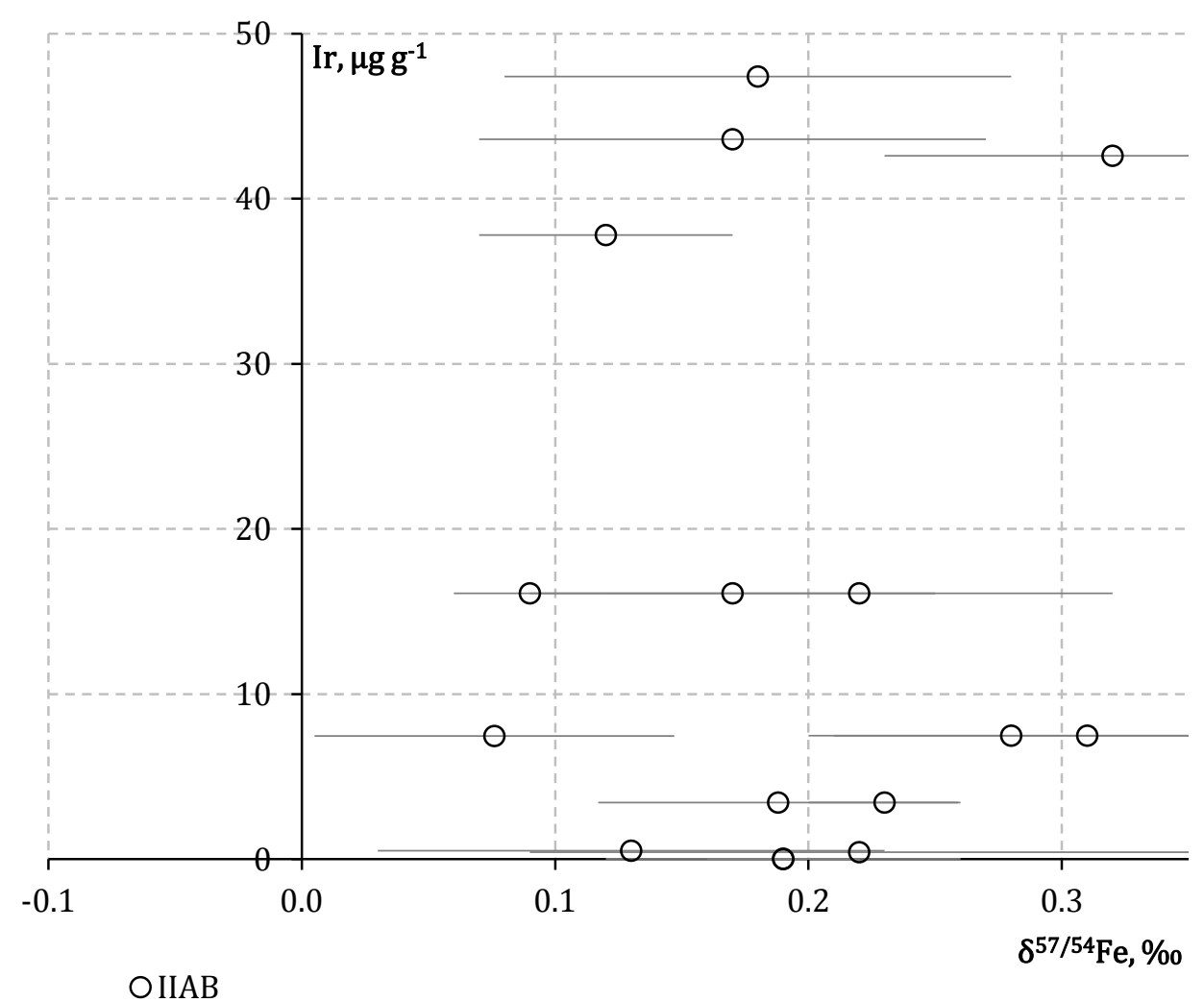

OIIAB 


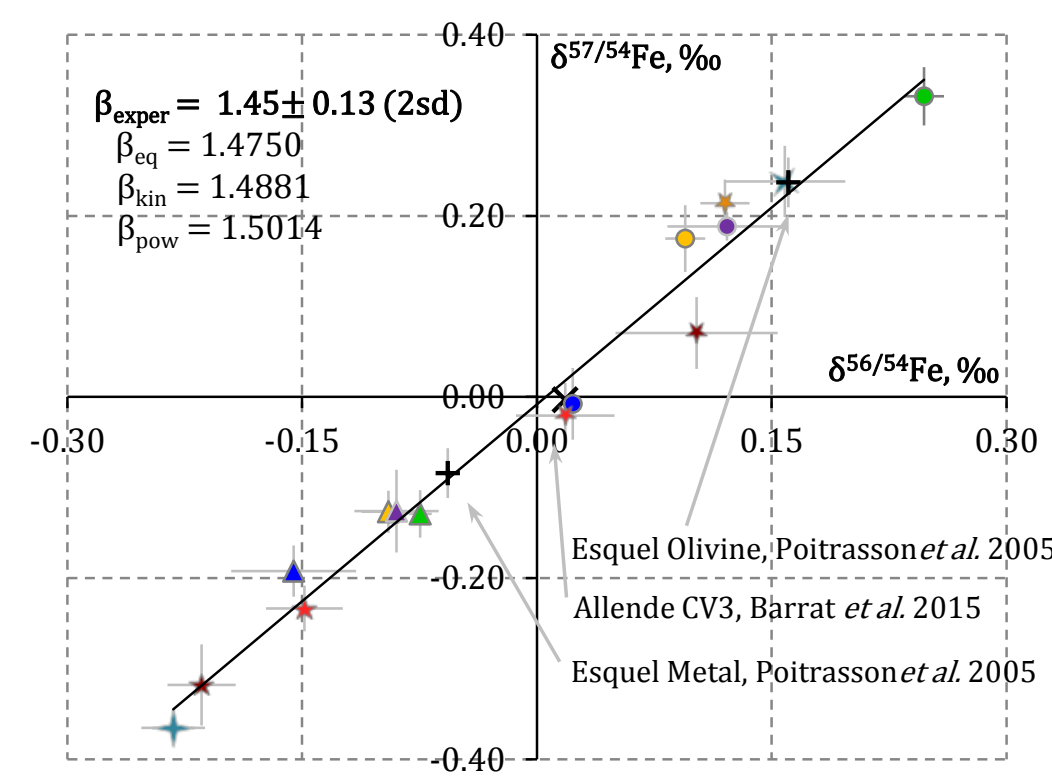

$\Delta$ Seymchan Olivine

$\triangle$ Fukang Olivine

$\triangle$ Esquel Olivine

$\triangle$ CMS-04071 Olivine

$\star$ EET-8750,51 Silicate

- Seymchan Metal

- Fukang Metal

- Esquel Metal

$\Delta_{\text {Met-Si }}{ }^{56 / 54}=0.178 \pm 0.092$

$\Delta_{\text {Met-Si }}{ }^{5 / 54}=0.322 \pm 0.033$

- CMS-04071 Metal $\quad \Delta_{\text {Met-Si }}^{56 / 54}=0.21 \pm 0.13$

*EET-8750,51 Metal $\Delta_{\text {Met-Si }{ }^{56 / 54}=0.32 \pm 0.15}$

$\star$ QUE-93001,23 Silicate $*$ QUE-93001,23 Metal $\Delta_{\text {Met-Si }}{ }^{56 / 54}=0.17 \pm 0.11$

+ Udei Station Silicate $\quad \times$ Udei Station Metal $\quad \Delta_{\text {Met-Si }}{ }^{56 / 54}=0.39 \pm 0.12$

* RKPA-7901 Metal

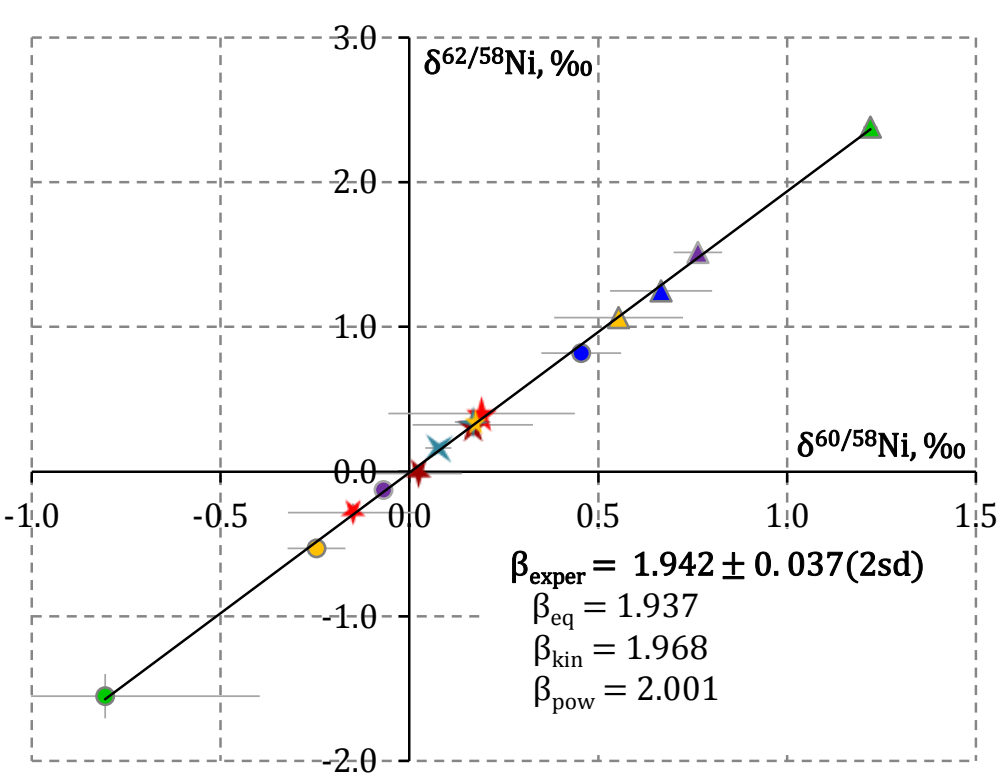

$\Delta$ Seymchan Olivine

$\triangle$ Fukang Olivine

$\triangle$ Esquel Olivine

$\triangle$ CMS-04071, olivine

* EET-8750,51 Silicate

- Seymchan Metal

- Fukang Metal

$\Delta_{\text {Met-Si }}{ }^{60 / 58}=-0.212 \pm 0.087$

- Esquel Metal

$\Delta_{\text {Met-Si }}{ }^{60 / 58}=-2.03 \pm 0.16$

* QUE-93001,23 Silicat

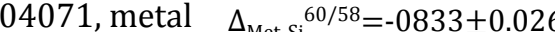

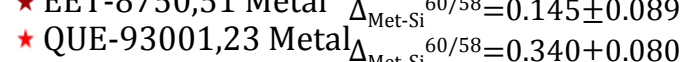

+ Udei Station, metal

* RKPA-79015, metal

$\times$ Udei Station, Silicate ${ }_{\Delta_{\text {Met-Si }}}{ }^{60 / 58}=0.091 \pm 0.018$ 

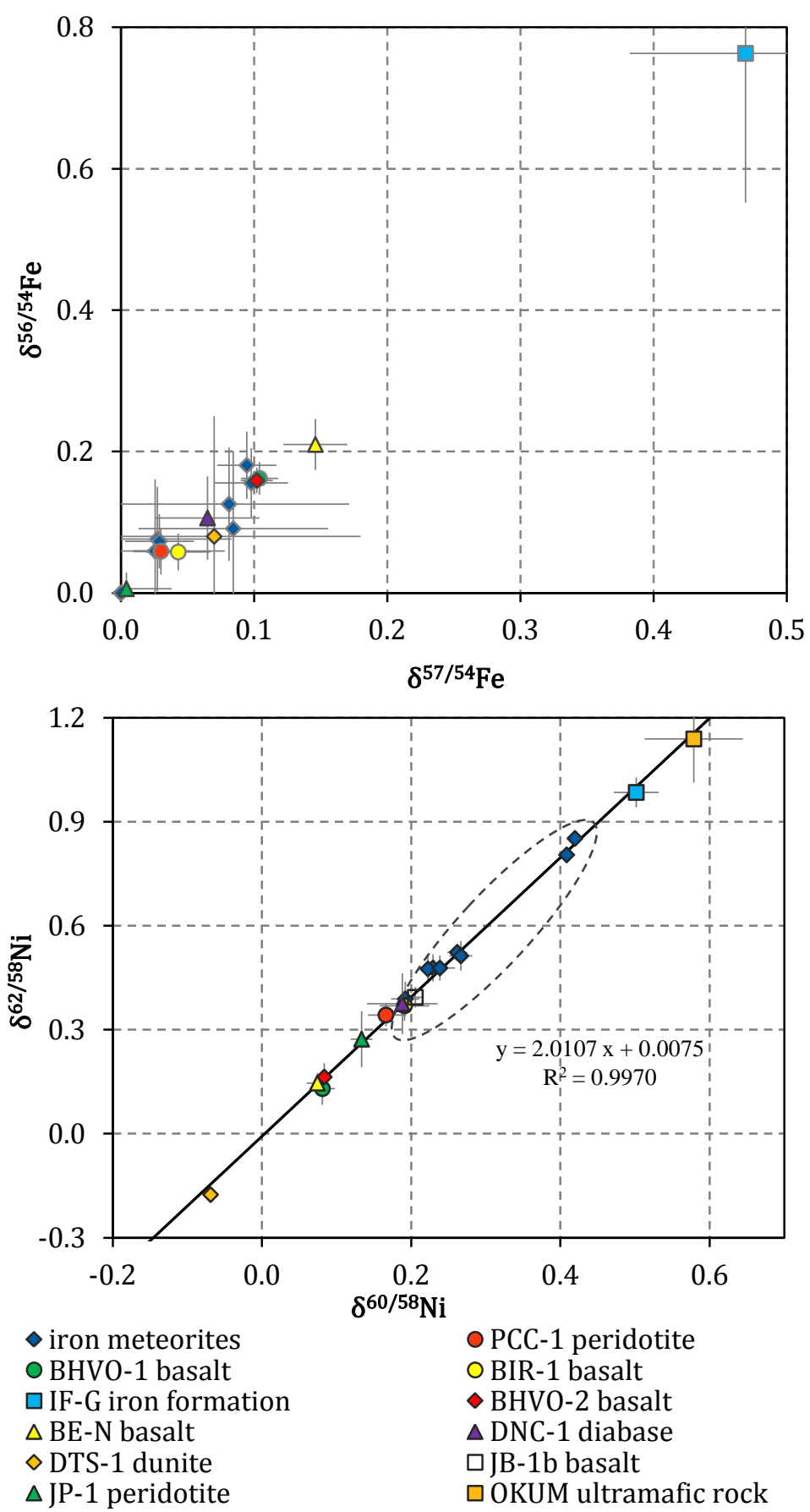


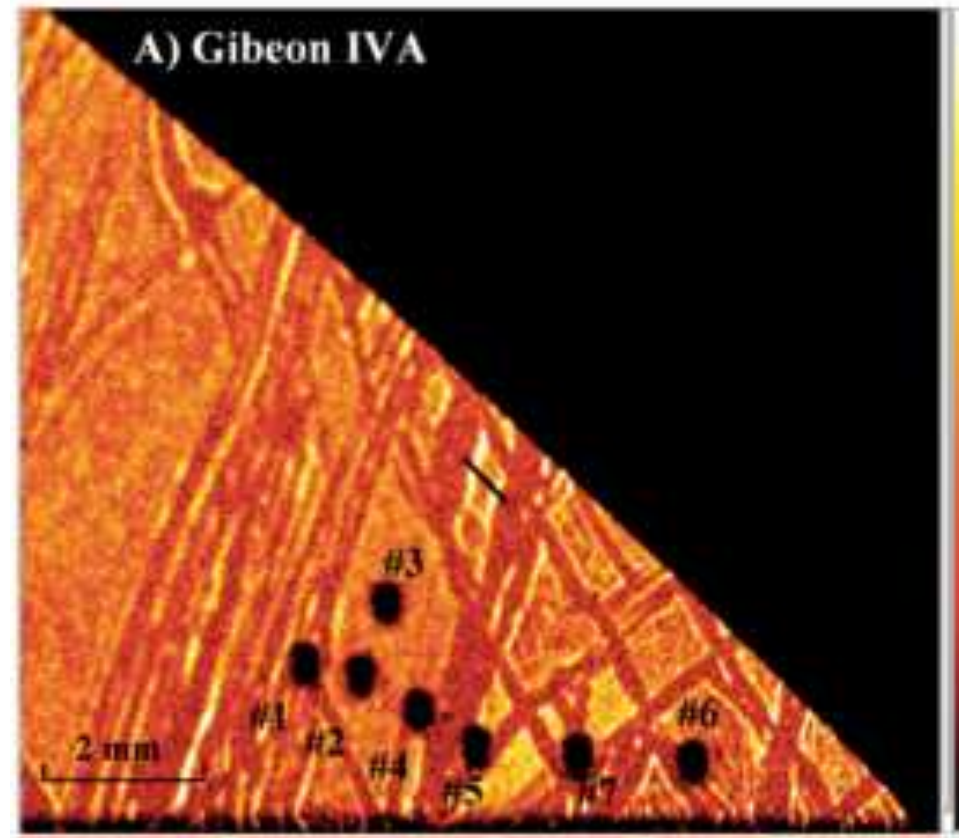

$-98$
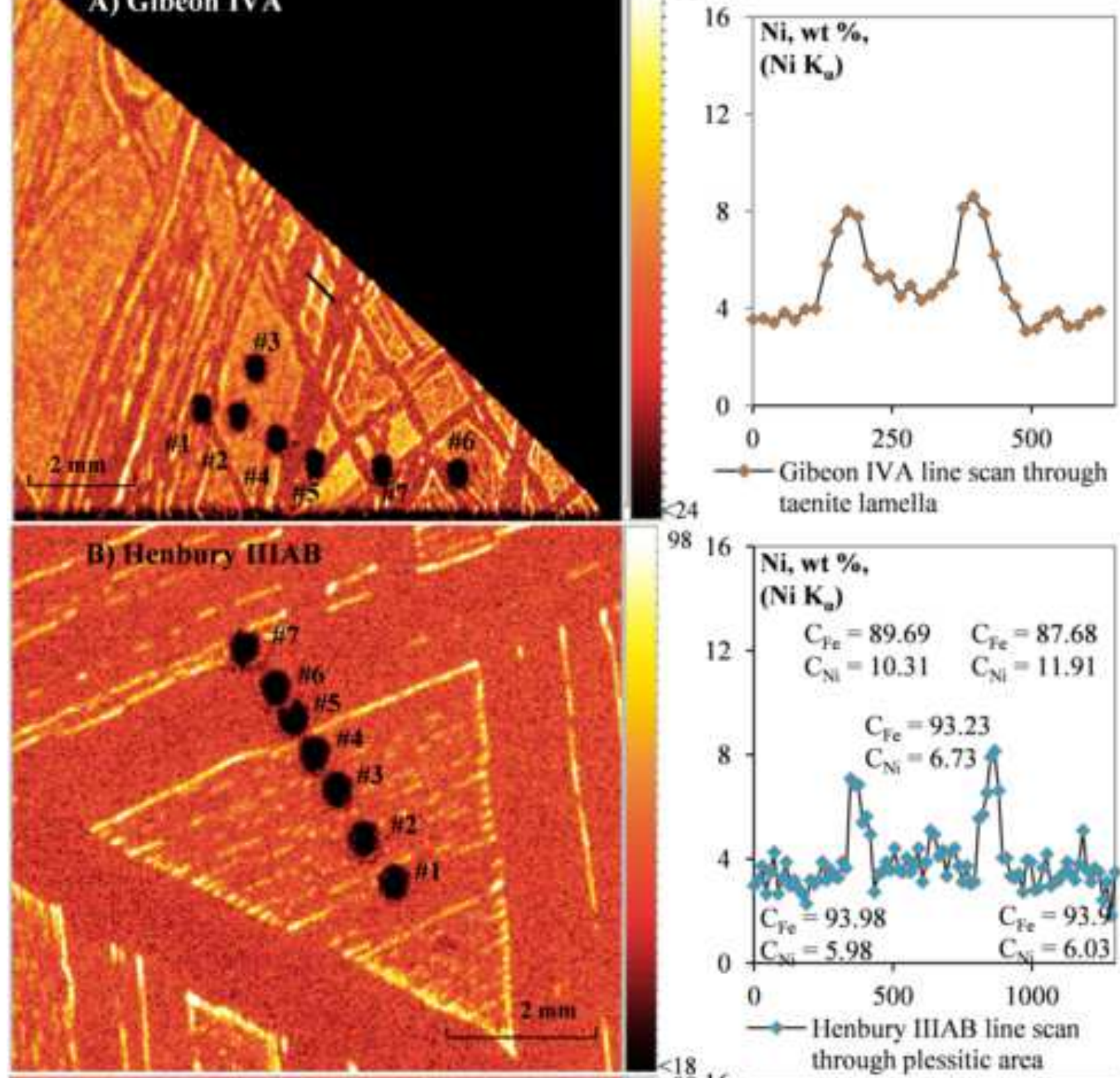

- - Gibeon IVA line scan through taenite lamella

9816

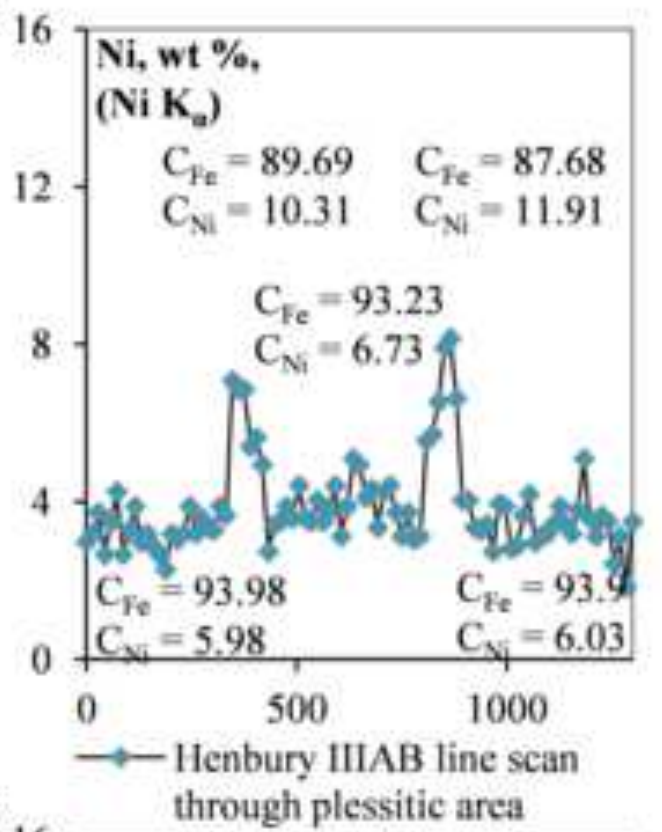

$<18$
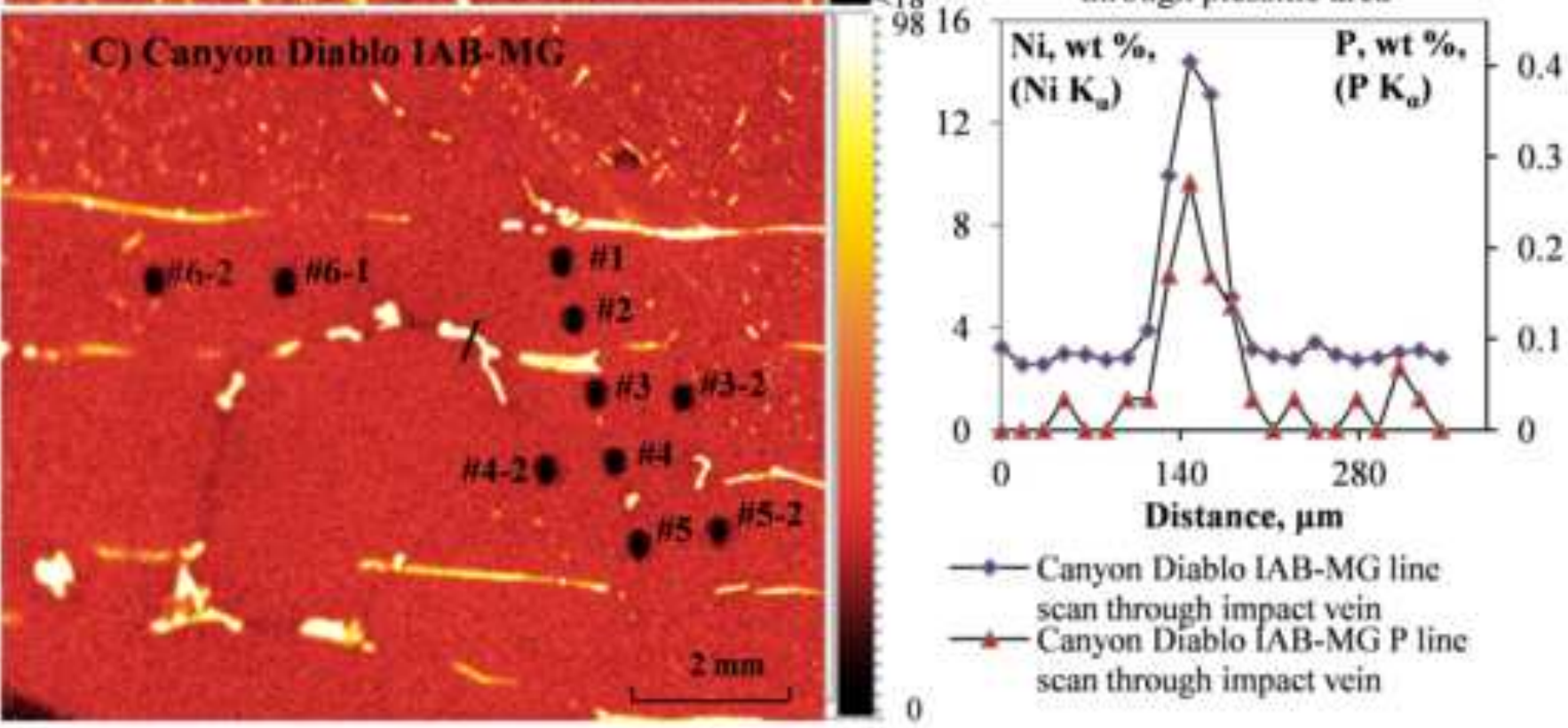


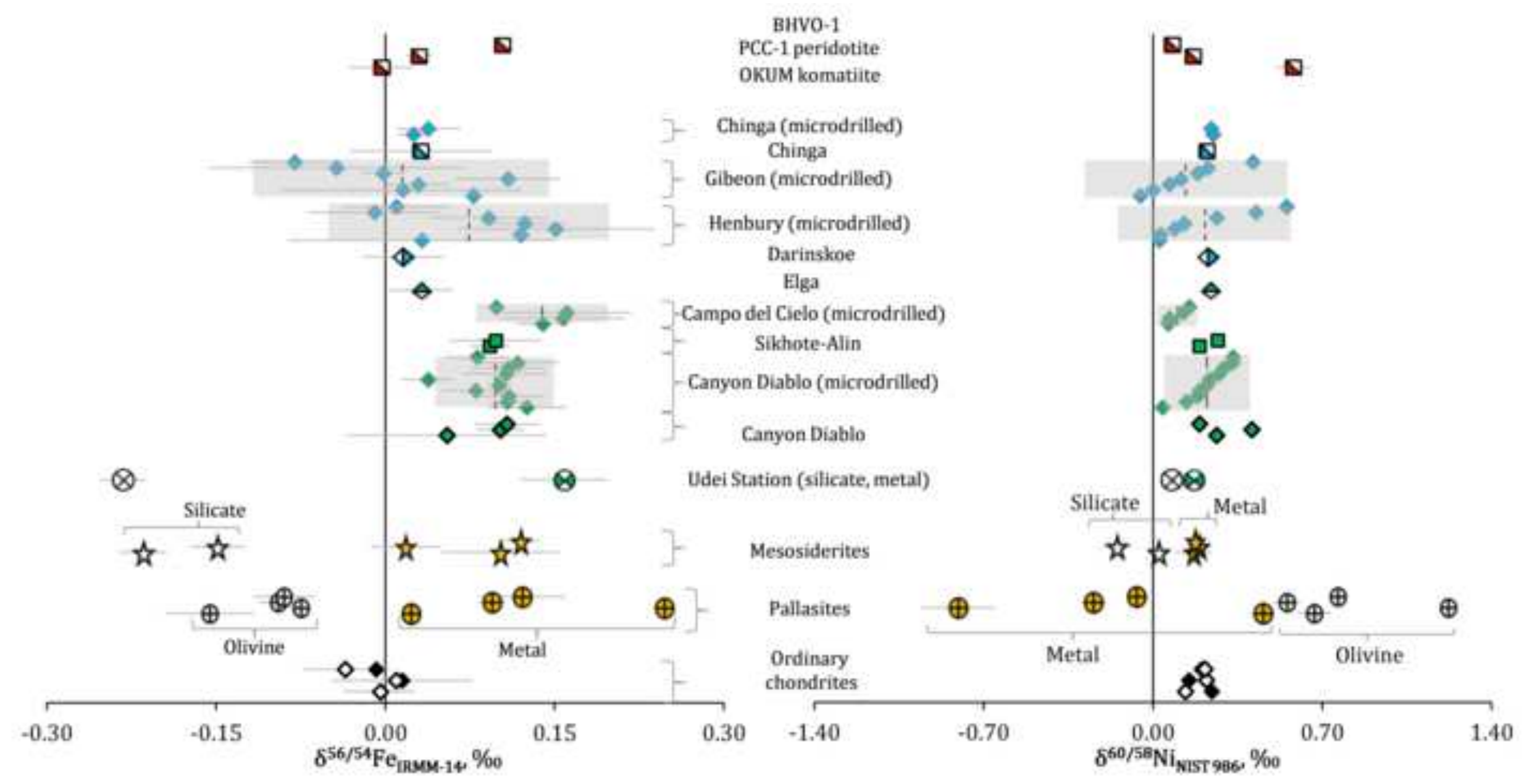



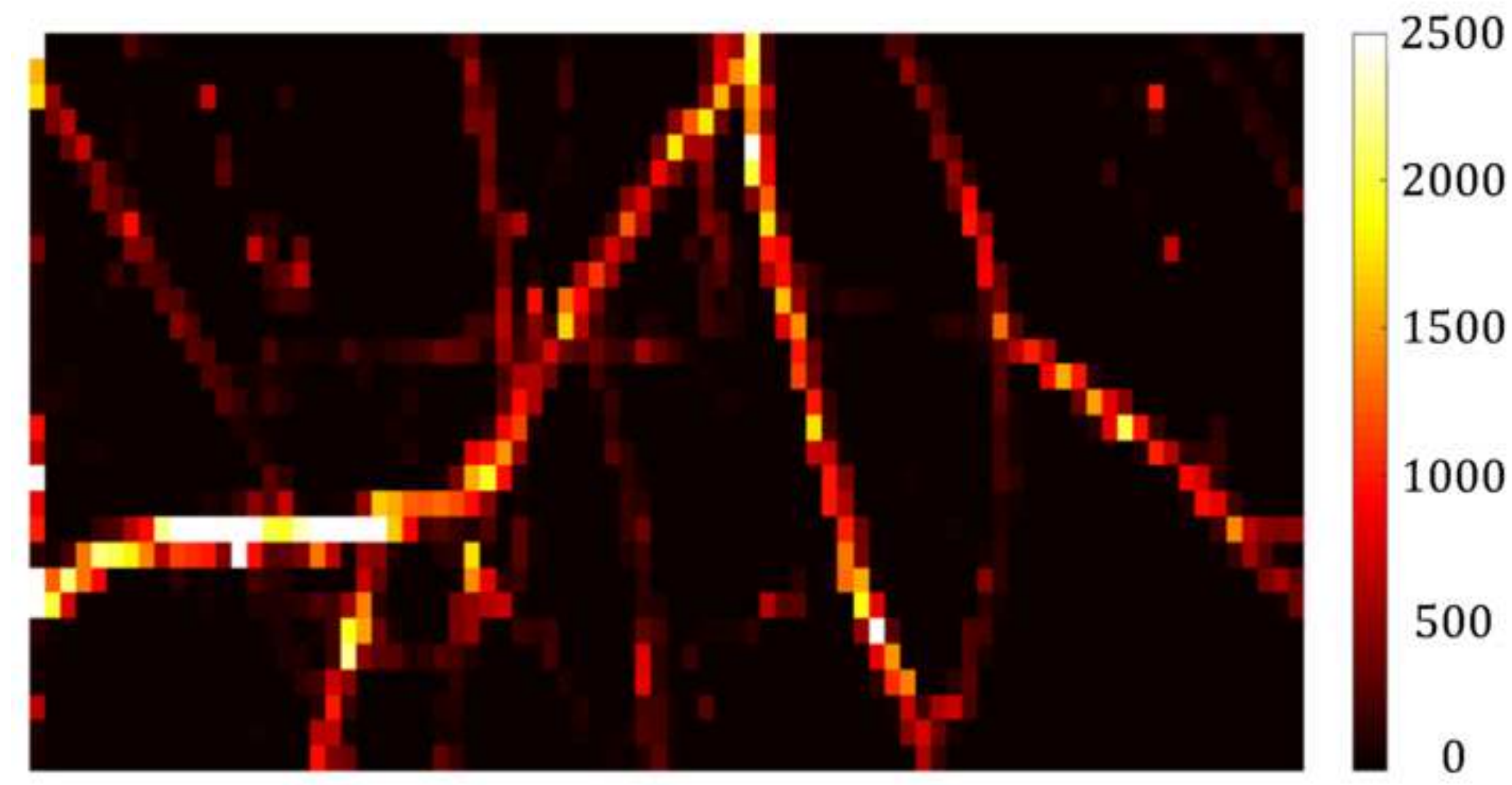

0 
Figure 6

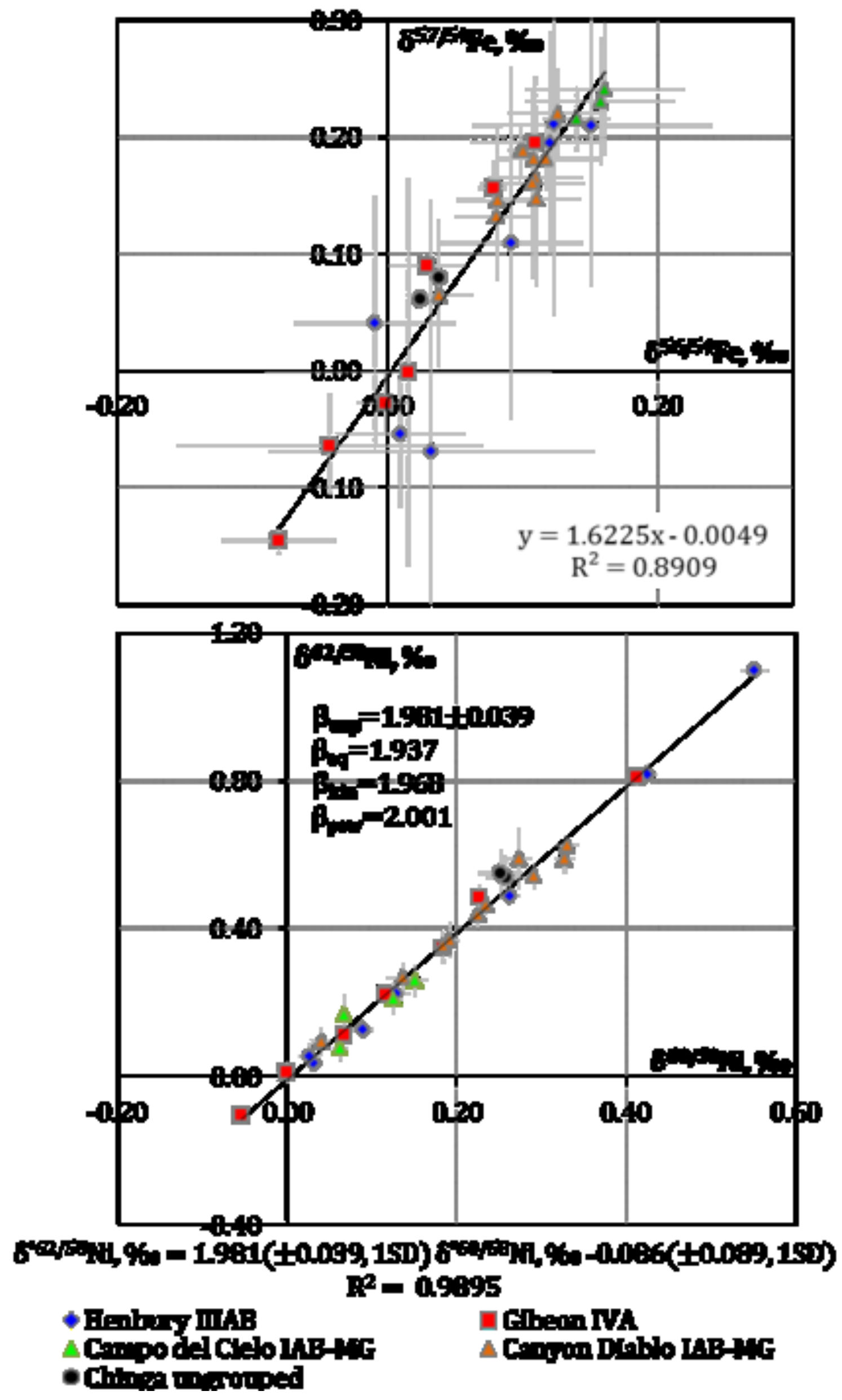

$$
\mathbf{R}^{2}=09895
$$

+ Einbor IIIA

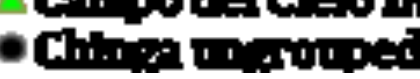

G.lbean IH

A Cran Dinblo IABATG 